\title{
Analysis of boundary layer effects due to usual boundary conditions or geometrical defects in elastic plates under bending: an improvement of the Love-Kirchhoff model.
}

\author{
Andrés León Baldelli · Jean-Jacques Marigo · Catherine Pideri
}

version of 26 March 2020

\begin{abstract}
We propose a model of flexural elastic plates accounting for boundary layer effects due to the most usual boundary conditions or to geometrical defects, constructed via matched asymptotic expansions. In particular, considering a rectangular plate clamped at two opposite edges while the other two are free, we derive the effective boundary conditions or effective transmission conditions that the two first terms of the outer expansion must satisfy. The new boundary value problems thus obtained are studied and compared with the classical Love-Kirchhoff plate model. Two examples of application illustrate the results.
\end{abstract}

Keywords Dimension reduction · Plate theory · Boundary layers · Matched asymptotic expansions

Mathematics Subject Classification (2000) MSC 35C20 - MSC 35J20 - MSC 74G10 - MSC 74Q15 - MSC 74B05 - MSC 74A40 - MSC 74K99

\section{Introduction}

Motivated by the sake to have access to good approximations of displacement and stress fields without numerically solving the full three dimensional problem, the (linear) Love-Kirchhoff theory of plates has been progressively constructed [31], studied, validated [37,42], and mathematically justified [10,11] for thin elastic structures in a small displacement setting. All these approaches take advantage of the presence of the small parameter $\eta$, that is the ratio between the thickness $h$ of the plate and its two other dimensions, to postulate or deduce the orders of magnitude of the different components of the stress tensor or some kinematic properties of the displacements. That allows to reduce the problem by one spatial dimension, the reduced problem being posed on the mid-surface $\Omega_{0}$ (whose unit vectors are $\mathbf{e}_{1}$ and $\mathbf{e}_{2}$ ) of the $3 \mathrm{D}$ structure $\Omega_{R}$. The counterpart is that one obtains a fourth order boundary value problem in terms of the deflection (that is the displacement in the direction $\mathbf{e}_{3}$ ) instead of a second order boundary value problem for the displacements in the full 3D setting. Specifically, in the case of a homogeneous plate made of an isotropic material and submitted to a system of external forces inducing a pure bending response, the deflection $U$ is governed by the well known bilaplacian operator

$$
\frac{\mathrm{E} h^{3}}{12\left(1-\nu^{2}\right)} \Delta^{2} U-\mathcal{F}=0 \quad \text { in } \quad \Omega_{0}
$$

where $\mathrm{E}$ and $\nu$ are Young's modulus and Poisson's ratio of the material, $\mathcal{F}$ the effective normal pressure resulting from body forces and surface tractions applied to the upper and lower faces. One must add two boundary conditions at each point of the boundary $\partial \Omega_{0}$ of the mid-plane so that the reduced problem is well-posed, like $U=\partial U / \partial n=0$ in the case of a clamped boundary. Those "reduced" boundary conditions must be themselves deduced from the $3 \mathrm{D}$ problem. It is for example the case for the clamped conditions which can be deduced from the 3D Dirichlet

A. León Baldelli

IMSIA, 91120 Palaiseau, France

E-mail: leon.baldelli@cnrs.fr

J.-J. Marigo

LMS, École Polytechnique, 91120 Palaiseau, France

E-mail: marigo@lms.polytechnique.fr

C. Pideri

Institut de Mathématiques Appliquées, Université de Toulon et du Var, France

E-mail: pideri@univ-tln.fr 
condition $\mathbf{u}=\mathbf{0}$ on the lateral boundary of $\Omega_{R}$. On this topic, and on the apparent disagreement between the order of equation (1) and the number of conditions that may be prescribed on the plate contour as well as on the interpretation of different types of (natural) boundary conditions that can occur in reduced theories, we refer the interested reader to the scientific discussion that can be traced back to the first statement of the bending problem for a plate by Poisson [39] and still is a topic of debate, see, e.g., [44,45] and [19]. Once the mathematical framework to justify a such asymptotic model by dimension reduction was well established, it was possible to enlarge the domain of validity of Love-Kirchhoff model, as done in [3] in a dynamical setting, or to justify other models like the Von Karman model in the large displacement setting, see [10] and references therein.

Thus, the Love-Kirchhoff model can be considered as a good approximation, at first order with respect to the small parameter, of the response of the 3D structure. However, if one considers the proof of the convergence result [10], it appears that the convergence holds in the sense of the $H^{1}\left(\Omega_{R}\right)$ norm for displacements and of the $L^{2}\left(\Omega_{R}\right)$ for stresses. That means that real stresses can be locally poorly approximated by the Love-Kirchhoff model. In particular, this is automatically the case near the boundary where Dirichlet conditions are prescribed, if the Poisson ratio is not zero. Indeed, in such a case, a singularity occurs at the corner between the lateral boundary and the upper or lower faces of $\Omega_{R}$. There, stresses are infinite which the Love-Kirchhoff model does not predict. In those regions, the problem becomes three-dimensional and one must introduce boundary layer correctors. This is also the case when the plate contains a transverse crack. Specifically, if the 3D structure contains a crack whose normal vector is in the plane $\left(\mathbf{e}_{1}, \mathbf{e}_{2}\right)$, whose depth in the direction $\mathbf{e}_{3}$ is $h / 2$, and whose length is comparable to the size of the plate, then the equation governing the deflection remains (1). In the Love-Kirchhoff model, such a defect is ignored, only cracks through the entire thickness of the plate are considered. A hint in this direction, as highlighted in [18], is that the model allows a plate to sustain singular shear stresses in the interior of the plate, owing to a static (though not kinematic) equivalence between smooth and singular loading systems. So, with this model, not only the singularity of stresses is not accounted for, but it is even impossible to estimate the risk of crack propagation. The main reason is that the intensity of stresses is strongly perturbed but only locally, like for Dirichlet boundary conditions. There again, the model must be improved by considering the boundary layer effects due to the crack. In terms of energy, those corrections are of the order $\eta$ compared to the leading term given by the Love-Kirchhoff model (essentially because the depth of the boundary layer is of the order the thickness $h$ ). So, in that sense, the estimate of the energy contained in the boundary layer effects should be the first term to be considered to improve the plate theory. But, in fact, the majority of the efforts have been devoted to improve the approximations of stresses in the bulk and not at those boundaries. Indeed, several works following the ideas elaborated in $[36,41]$ have had and still have for goal to relax the kinematic constraint on the rotation of the sections existing in the Love-Kirchhoff model to better approximate the transverse shear stresses $\sigma_{13}$ and $\sigma_{23}$. This leads to the so-called Mindlin-Reissner plate theory (with all its variants) $[5,24,32,40,43]$. It turns out that, for thin plates, it leads to a correction in the energy estimate of the order $\eta^{2}$, hence at the next order with regards to boundary layer effects. Indeed, its failure to correctly capture the behaviour near the boundary is apparent in the slow rate of convergence of the approximate to the full three-dimensional elastic solution, see, e.g., the review paper [14] and numerical computations in [4] which compare several enriched, or otherwise corrected, reduced models.

Paradoxically, few efforts have focused on the construction of a theory of plates enriched with boundary layer effects, at least in a systematic way. Indeed, substantial work has involved studying boundary layers themselves [8, $12,16,21,23,27,30]$ but not to construct a boundary value problem for the whole plate which includes the information coming from the boundary layers. And yet, the tools to carry out such a task exist and have already been used in contexts other than those of the plate theory, like in $[1,2,6,7,15,20,25,35]$ where effective models are constructed for 3D elastic (homogeneous or composite) bodies containing defects (cracks, voids, inclusions,...) periodically distributed near a surface. In such situations, the presence of the defects induces boundary layer effects which finally perturbs the overall fields (at second order in terms of the potential energy). To account for their contribution, the method of matched asymptotic expansions $[17,26,28]$ can be used to have a good approximation of the fields in the region of the defects (inner expansions) and to obtain effective boundary conditions on, or effective transmission conditions across, the "surface of defects" for the boundary value problem posed on the whole body (outer expansions). More recently, the method has been also used in a dynamical context to study the influence of such surface defects on the propagation of waves in metamaterials [33,34,38]. One obtains a so-called effective boundary value problem whose solution constitutes a good approximation of the real solution up to second order. This boundary value problem contains effective boundary conditions or effective transmission conditions which can be of Robin's or Ventcel's type $[9,29,46]$ with coefficients that are obtained from a few boundary layer problems posed at the scale of the defects. In all cases, those effective boundary or transmission conditions have an energetic interpretation. Specifically, it is possible to show that the effective boundary value problem is equivalent to find the extrema of an effective potential energy which contains surface terms characterising boundary layer effects due to the defects. However, the coefficients entering in the definition of those surface energies can have any sign, rendering delicate the mathematical analysis of the effective boundary layer problem, see $[15,35]$. 
In the present paper, an asymptotic procedure is followed in order to construct an effective model of plate accurate up to the second order and hence improving the Love-Kirchhoff model by including boundary layer effects. However, since the implementation of the matched asymptotic expansion method coupling dimension reduction and boundary layers is rather intricate, requiring to consider terms up to the fourth order for the inner asymptotic expansions of the displacements, its construction is only achieved in a limited setting. Specifically, it is assumed first that the 3D body is made of an elastic isotropic homogeneous material, then that the geometry of the defects and the loading of the plate have symmetry properties so that the response corresponds to pure bending without membrane coupling. Moreover, still for the sake of simplicity of presentation, it is assumed that the body has straight lateral boundaries and contains a geometrical defect which is straight and unidirectional (i.e. , with a cylindrical shape). As far as boundary conditions are concerned, we consider here only the cases of clamped and free boundaries. The study of boundary layer effects associated with other types of boundary conditions (like simply supported) is left to future works. We claim that the development of the method in this restricted context is nevertheless sufficient to exhibit the improvements made to the Love-Kirchhoff model. Specifically, the paper is organised as follows. In Section 2, first are made the main assumptions and set the 3D problem. Then are derived the equations that the first terms of the outer expansions of the displacement and stress fields (in particular the deflections $U^{0}$ and $U^{1}$, the associated bending moments $\mathcal{M}^{0}$ and $\mathcal{M}^{1}$, and the associated shear forces $\mathcal{T}^{0}$ and $\mathcal{T}^{1}$ ) must satisfy far from the lateral boundaries and the line of defect. In Section 3 we study the boundary layer near the clamped sides to obtain the effective kinematic boundary conditions that $U^{0}$ and $U^{1}$ must satisfy on those sides. In Section 4 we study the boundary layer near the free sides to infer the effective static boundary conditions that $\mathcal{M}^{0}, \mathcal{M}^{1}, \mathcal{T}^{0}$, and $\mathcal{T}^{1}$ must satisfy on those sides. In Section 5 we study the boundary layer due to the geometrical defect located near the plane $x_{2}=0$ of the body to derive the effective kinematic and static transmission conditions that $U^{0}, U^{1}, \mathcal{M}^{0}, \mathcal{M}^{1}, \mathcal{T}^{0}$, and $\mathcal{T}^{1}$ must satisfy across the line of defect. In Section 6 we study the two boundary value problems thus constructed for $U^{0}$ and $U^{1}$. Then these two problems are merged to propose a single boundary value problem of which is given an energetic interpretation and which could constitute the prototype of an improved Love-Kirchhoff model. Finally, two examples of application are given in Section 7.

Throughout the paper, Greek indices run from 1 to 2 whereas Latin indices run from 1 to 3 . The summation convention on repeated indices is already assumed. Vectors and second order tensors are indicated by bold face letters. The inner product is denoted by a dot. In general (but with some exceptions, like $\nu$ ), material parameters or effective coefficients are indicated by sans serif letters. As far as the orders of magnitude with respect to the small parameter $\eta$ are concerned, we use the following terminology: a term is said of order $p, p \in \mathbb{R}$, when it is of the order of $\eta^{p}$; occasionally a term is said of the first order (resp. second order) when it is of the order of $\eta^{0}$ (resp. $\left.\eta^{1}\right)$.

\section{Problem setting for a plate without defects}

\subsection{The three-dimensional problem}

We consider a rectangular plate whose reference configuration is the following domain $\Omega_{R}$ of $\mathbb{R}^{3}$

$$
\Omega_{R}=(-L / 2,+L / 2) \times(-\ell / 2,+\ell / 2) \times(-h / 2,+h / 2) .
$$

The geometrical defect will be introduced in Section 5. The plate is made of an elastic isotropic material whose Young modulus and Poisson ratio are respectively $\mathrm{E}$ and $\nu$. The sides $x_{1}= \pm L / 2$ are clamped whereas the sides $x_{2}= \pm \ell / 2$ are free. The upper and lower faces $x_{3}= \pm h / 2$ are submitted to the same density of normal surface forces $\mathbf{F}: \mathbf{F}\left(x_{1}, x_{2}\right)=F_{\mathrm{R}}\left(x_{1}, x_{2}\right) \mathbf{e}_{3}$. Moreover the plate is submitted to body forces oriented following $\mathbf{e}_{3}$ and whose density $\mathbf{f}$ is assumed to be symmetric with respect to the mid-plane $x_{3}=0$, i.e. ,

$$
\mathbf{f}(\mathbf{x})=f_{\mathrm{R}}(\mathbf{x}) \mathbf{e}_{3} \quad \text { with } \quad f_{\mathrm{R}}\left(x_{1}, x_{2},-x_{3}\right)=f_{\mathrm{R}}\left(x_{1}, x_{2},+x_{3}\right) .
$$

The surface and body forces densities are assumed to be smooth functions of the coordinates so that, in a small displacement setting, the displacement field $\mathbf{u}$ and the stress field $\boldsymbol{\sigma}$ at equilibrium are the unique solution of the following boundary value problem

\begin{tabular}{cccc}
\hline$(A)$ in $\Omega_{R}$ & $(B)$ on $x_{1}= \pm L / 2$ & $(C)$ on $x_{2}= \pm \ell / 2$ & $(D)$ on $x_{3}= \pm h / 2$ \\
\hline$\left\{\begin{array}{l}\operatorname{div} \boldsymbol{\sigma}+f_{\mathrm{R}} \mathbf{e}_{3}=\mathbf{0} \\
\mathrm{E} \boldsymbol{\varepsilon}(\mathbf{u})=-\nu \operatorname{Tr} \boldsymbol{\sigma} \mathbf{I}+(1+\nu) \boldsymbol{\sigma}\end{array}\right.$ & $\mathbf{u}=\mathbf{0}$ & $s \boldsymbol{\sigma} \mathbf{e}_{2}=\mathbf{0}$ & $\boldsymbol{\sigma} \mathbf{e}_{3}=F_{\mathrm{R}} \mathbf{e}_{3}$ \\
$2 \varepsilon(\mathbf{u})=\nabla \mathbf{u}+\nabla^{T} \mathbf{u}$ & & \\
\hline
\end{tabular}


By virtue of the symmetry of the loading, it is easy to see that the in-plane components $u_{\alpha}, \alpha \in\{1,2\}$, of the displacement are odd functions of $x_{3}$ whereas the out-of-plane component $u_{3}$ is an even function of $x_{3}$ :

$$
u_{\alpha}\left(x_{1}, x_{2},-x_{3}\right)=-u_{\alpha}\left(x_{1}, x_{2},+x_{3}\right), \quad u_{3}\left(x_{1}, x_{2},-x_{3}\right)=+u_{3}\left(x_{1}, x_{2},+x_{3}\right) .
$$

In the same manner, one obtains that $\sigma_{\alpha \beta}$ are odd functions of $x_{3}$ whereas $\sigma_{\alpha 3}$ are even functions of $x_{3}$. We will also use in the sequel the classical resultant quantities like shear forces and bending moments which, by virtue of the symmetries, are defined as follows

$$
\mathcal{T}_{\alpha}\left(x_{1}, x_{2}\right)=2 \int_{0}^{h / 2} \sigma_{\alpha 3}(\mathbf{x}) d x_{3}, \quad \mathcal{M}_{\alpha \beta}\left(x_{1}, x_{2}\right)=-2 \int_{0}^{h / 2} x_{3} \sigma_{\alpha \beta}(\mathbf{x}) d x_{3},
$$

whereas the in-plane resultant forces $\mathcal{N}_{\alpha \beta}$ vanish by symmetry: $\mathcal{N}_{\alpha \beta}\left(x_{1}, x_{2}\right):=\int_{-h / 2}^{h / 2} \sigma_{\alpha \beta}(\mathbf{x}) d x_{3}=0$.

\subsection{Asymptotic assumptions}

The plate thickness $h$ is supposed small compared to the two other dimensions $L$ and $\ell$ and the ratio $\eta=h / L$ is a small dimensionless parameter. After introducing the dimensionless coordinates $\overline{\mathbf{x}}=\left(x_{1} / L, x_{2} / L\right)$ and $y_{3}=x_{3} / h$, a material point of the plate is defined by $\left(\overline{\mathbf{x}}, y_{3}\right)$ and lays in the fix domain $\Omega=\Omega_{0} \times(-1 / 2,+1 / 2)$ where $\Omega_{0}$ is the dimensionless mid-plane,

$$
\Omega_{0}=(-1 / 2,+1 / 2) \times(-\bar{\ell} / 2,+\bar{\ell} / 2), \quad \bar{\ell}=\frac{\ell}{L} .
$$

Taking $L$ as the reference length, the displacement field $\mathbf{u}$ solution of the boundary value problem reads

$$
\mathbf{u}(\mathbf{x})=L \mathbf{u}^{\eta}\left(\overline{\mathbf{x}}, y_{3}\right)
$$

where the dependence on the small parameter $\eta$ is now explicit. For stresses, we take $E h / L$ as the reference pressure so that the stress field at equilibrium $\sigma$ reads

$$
\boldsymbol{\sigma}(\mathbf{x})=\eta \mathrm{E} \boldsymbol{\sigma}^{\eta}\left(\overline{\mathbf{x}}, y_{3}\right) .
$$

In (3) and (4), the new unknown displacement and stress fields $\mathbf{u}^{\eta}$ and $\boldsymbol{\sigma}^{\eta}$ are dimensionless. The main goal of the paper is to construct an approximation of the rescaled solution $\left(\mathbf{u}^{\eta}, \boldsymbol{\sigma}^{\eta}\right)$ by using asymptotic expansions with respect to the small parameter $\eta$. For that, the first step consists in defining the order of magnitude of the loading, i.e. , of body and surface forces $f_{\mathrm{R}}$ and $F_{\mathrm{R}}$, with respect to $\eta$. The choice of the order is such that the leading term of the displacement expansion is of order 0. (Moreover, we will see that the stress expansion starts also at order 0 by virtue of the chosen normalization (4).) For that, it turns out that $f_{\mathrm{R}}$ must be of order 2 and $F_{\mathrm{R}}$ of order 3 . Accordingly, we assume that $f_{\mathrm{R}}$ and $F_{\mathrm{R}}$ can read as

$$
f_{\mathrm{R}}(\mathbf{x})=\frac{\eta^{2} \mathrm{E}}{L} f\left(\overline{\mathbf{x}}, y_{3}\right), \quad F_{\mathrm{R}}\left(x_{1}, x_{2}\right)=\eta^{3} \mathrm{E} F(\overline{\mathbf{x}}),
$$

where the densities $f$ and $F$ are dimensionless fields independent of $\eta$. Let us note that, because the problem is linear, that choice is not really important because it is always possible to be in this situation after a suitable rescaling of the forces. Finally, the rescaled fields $\mathbf{u}^{\eta}$ and $\boldsymbol{\sigma}^{\eta}$ are the unique solution of the following boundary value problem which is posed on the upper half of the rescaled domain, by virtue of the assumed symmetry properties:

$$
\begin{aligned}
& \text { Equilibrium equation: } \eta \frac{\partial \sigma_{\alpha \beta}^{\eta}}{\partial \bar{x}_{\beta}}+\frac{\partial \sigma_{\alpha 3}^{\eta}}{\partial y_{3}}=0, \quad \eta \frac{\partial \sigma_{3 \beta}^{\eta}}{\partial \bar{x}_{\beta}}+\frac{\partial \sigma_{33}^{\eta}}{\partial y_{3}}+\eta^{2} f=0 \text {; } \\
& \text { Constitutive relation: } \varepsilon^{\eta}=-\nu \operatorname{Tr} \boldsymbol{\sigma}^{\eta} \mathbf{I}+(1+\nu) \boldsymbol{\sigma}^{\eta} \text {; } \\
& \text { Compatibility relations: } 2 \varepsilon_{\alpha \beta}^{\eta}=\frac{1}{\eta} \frac{\partial u_{\alpha}^{\eta}}{\partial \bar{x}_{\beta}}+\frac{1}{\eta} \frac{\partial u_{\beta}^{\eta}}{\partial \bar{x}_{\alpha}}, \quad 2 \varepsilon_{\alpha 3}^{\eta}=\frac{1}{\eta} \frac{\partial u_{3}^{\eta}}{\partial \bar{x}_{\alpha}}+\frac{1}{\eta^{2}} \frac{\partial u_{\alpha}^{\eta}}{\partial y_{3}}, \quad \varepsilon_{33}^{\eta}=\frac{1}{\eta^{2}} \frac{\partial u_{3}^{\eta}}{\partial y_{3}} \text {; } \\
& \text { Boundary conditions: } \begin{cases}\mathbf{u}^{\eta}=\mathbf{0} & \text { on } \bar{x}_{1}= \pm 1 / 2, \\
\boldsymbol{\sigma}^{\eta} \mathbf{e}_{2}=\mathbf{0} & \text { on } \bar{x}_{2}= \pm \bar{\ell} / 2, \\
\boldsymbol{\sigma}^{\eta} \mathbf{e}_{3}=\eta^{2} F \mathbf{e}_{3} & \text { on } y_{3}=1 / 2, \\
u_{\alpha}^{\eta}=0, \quad \sigma_{33}^{\eta}=0 & \text { on } y_{3}=0,\end{cases}
\end{aligned}
$$

where we use implicit summation over repeated indices. Note that the relations (8) between the strains $\varepsilon^{\eta}$ and the gradient of the displacements are a consequence of the chosen normalizations. 
We also introduce dimensionless shear forces and bending moments and choose their normalization in such a way that their expansion eventually starts at order 0 . Specifically, we set

$$
\mathcal{T}\left(x_{1}, x_{2}\right)=\eta^{3} \mathrm{E} L \mathcal{T}^{\eta}(\overline{\mathbf{x}}), \quad \mathcal{M}\left(x_{1}, x_{2}\right)=\eta^{3} \mathrm{E} L^{2} \mathcal{M}^{\eta}(\overline{\mathbf{x}})
$$

which are related to the dimensionless stress field $\boldsymbol{\sigma}^{\eta}$ by

$$
\mathcal{T}_{\alpha}^{\eta}(\overline{\mathbf{x}})=\frac{2}{\eta} \int_{0}^{1 / 2} \sigma_{\alpha 3}^{\eta}\left(\overline{\mathbf{x}}, y_{3}\right) d y_{3}, \quad \mathcal{M}_{\alpha \beta}^{\eta}(\overline{\mathbf{x}})=-2 \int_{0}^{1 / 2} y_{3} \sigma_{\alpha \beta}^{\eta}\left(\overline{\mathbf{x}}, y_{3}\right) d y_{3} .
$$

The equilibrium equations satisfied by the dimensionless shear forces and bending moments are deduced from the local equilibrium equations (6). Specifically, multiplying the first equation of (6) by $y_{3}$, integrating with respect to $y_{3}$ over $(0,1 / 2)$, integrating by parts the term involving the shear stress, and using boundary conditions on $y_{3}=1 / 2$ leads to

$$
\frac{\partial \mathcal{M}_{\alpha \beta}^{\eta}}{\partial \bar{x}_{\beta}}+\mathcal{T}_{\alpha}^{\eta}=0 \quad \text { in } \Omega_{0}
$$

Furthermore, integrating the second equation of (6) with respect to $y_{3}$ over $(0,1 / 2)$ and using the boundary conditions on $y_{3}=0$ and $y_{3}=1 / 2$ gives

$$
\frac{\partial \mathcal{T}_{\alpha}^{\eta}}{\partial \bar{x}_{\alpha}}+\mathcal{F}^{0}=0 \quad \text { in } \Omega_{0}
$$

where

$$
\mathcal{F}^{0}(\overline{\mathbf{x}})=2 \int_{0}^{1 / 2} f\left(\overline{\mathbf{x}}, y_{3}\right) d y_{3}+2 F(\overline{\mathbf{x}})
$$

Inserting the shear force from (11) into (12), we obtain the second order partial differential equation governing the bending moments

$$
\frac{\partial^{2} \mathcal{M}_{\alpha \beta}^{\eta}}{\partial \bar{x}_{\alpha} \partial \bar{x}_{\beta}}-\mathcal{F}^{0}=0 \quad \text { in } \Omega_{0}
$$

2.3 Asymptotic expansions far from the sides of the plate

\subsubsection{Hypothesis on the form of the outer expansions}

We suppose that, far from the lateral boundaries of the plate, the displacement $\mathbf{u}^{\eta}$ admits the following asymptotic expansion with respect to the small parameter $\eta$

$$
\mathbf{u}^{\eta}\left(\overline{\mathbf{x}}, y_{3}\right)=\mathbf{u}^{0}\left(\overline{\mathbf{x}}, y_{3}\right)+\eta \mathbf{u}^{1}\left(\overline{\mathbf{x}}, y_{3}\right)+\eta^{2} \mathbf{u}^{2}\left(\overline{\mathbf{x}}, y_{3}\right)+\eta^{3} \mathbf{u}^{3}\left(\overline{\mathbf{x}}, y_{3}\right)+\cdots,
$$

where the different terms $\mathbf{u}^{i}$ of the expansion are to be determined. The intuition behind the expansion above is to construct a partial sum that approximates the solution $\mathbf{u}(\mathbf{x})$ to the system $(2)_{A}$ as well as the boundary conditions $(2)_{B, C, D}$, for small $\eta$ with increasing accuracy as the number of terms in the series increases. By linearity, the same applies for strains and stresses. Consequently, strains and stresses admit the same type of asymptotic expansion, except that their expansion starts a priori at order -2 instead of 0 . (We will show that they start in reality at order 0.) Specifically, one has

$$
\left\{\begin{array}{l}
\varepsilon^{\eta}\left(\overline{\mathbf{x}}, y_{3}\right)=\eta^{-2} \varepsilon^{-2}\left(\overline{\mathbf{x}}, y_{3}\right)+\eta^{-1} \boldsymbol{\varepsilon}^{-1}\left(\overline{\mathbf{x}}, y_{3}\right)+\varepsilon^{0}\left(\overline{\mathbf{x}}, y_{3}\right)+\eta \varepsilon^{1}\left(\overline{\mathbf{x}}, y_{3}\right)+\cdots \\
\boldsymbol{\sigma}^{\eta}\left(\overline{\mathbf{x}}, y_{3}\right)=\eta^{-2} \boldsymbol{\sigma}^{-2}\left(\overline{\mathbf{x}}, y_{3}\right)+\eta^{-1} \boldsymbol{\sigma}^{-1}\left(\overline{\mathbf{x}}, y_{3}\right)+\boldsymbol{\sigma}^{0}\left(\overline{\mathbf{x}}, y_{3}\right)+\eta \boldsymbol{\sigma}^{1}\left(\overline{\mathbf{x}}, y_{3}\right)+\cdots
\end{array}\right.
$$

Moreover, by (10), shear forces admit an asymptotic expansion which starts at order -3 whereas the asymptotic expansion of the bending moments starts at order -2

$$
\left\{\begin{array}{l}
\mathcal{T}^{\eta}(\overline{\mathbf{x}})=\eta^{-3} \mathcal{T}^{-3}(\overline{\mathbf{x}})+\eta^{-2} \mathcal{T}^{-2}(\overline{\mathbf{x}})+\eta^{-1} \mathcal{T}^{-1}(\overline{\mathbf{x}})+\mathcal{T}^{0}(\overline{\mathbf{x}})+\eta \mathcal{T}^{1}(\overline{\mathbf{x}})+\cdots \\
\mathcal{M}^{\eta}(\overline{\mathbf{x}})=\eta^{-2} \mathcal{M}^{-2}(\overline{\mathbf{x}})+\eta^{-1} \mathcal{M}^{-1}(\overline{\mathbf{x}})+\mathcal{M}^{0}(\overline{\mathbf{x}})+\eta \mathcal{M}^{1}(\overline{\mathbf{x}})+\cdots
\end{array}\right.
$$




\subsubsection{Determination of the form of the first terms of the displacements, strains and stresses expansions}

Inserting these asymptotic expansions into the equations (6)-(9) of the boundary value problem gives a sequence of equations at successive orders that we introduce and use below.

(1) Equilibrium equations, constitutive relations and compatibility conditions at order -2 . Equilibrium equation (6) at order -2 gives $\sigma_{i 3,3}^{-2}=0$ and hence $\sigma_{i 3}^{-2}$ does not depend on $y_{3}$. Since $\sigma_{i 3}^{-2}$ vanishes at $y_{3}=1 / 2$ by $(9)$, one has ${\sigma_{i 3}^{-2}}^{-2} 0$ everywhere. Then, since $\varepsilon_{\alpha \beta}^{-2}=0$ by (8), one deduces from (7) that $\sigma_{\alpha \beta}^{-2}=0$ and hence $\boldsymbol{\sigma}^{-2}=\mathbf{0}$. Consequently, $\varepsilon^{-2}=\mathbf{0}$ and (8) gives $u_{i, 3}^{0}=0$ and hence $\mathbf{u}^{0}$ does not depend on $y_{3}$. Since $u_{\alpha}^{0}=0$ on $y_{3}=0$ we get $u_{\alpha}^{0}=0$ everywhere. Thus, at this step one has obtained

$$
\mathbf{u}^{0}\left(\overline{\mathbf{x}}, y_{3}\right)=U^{0}(\overline{\mathbf{x}}) \mathbf{e}_{3}, \quad \boldsymbol{\sigma}^{-2}=\mathbf{0}, \quad \varepsilon^{-2}=\mathbf{0},
$$

where the deflection at order $0, U^{0}(\overline{\mathbf{x}})$, has to be determined.

(2) Equilibrium equations, constitutive relations and compatibility conditions at order -1 . Owing to $\boldsymbol{\sigma}^{-2}=\mathbf{0}$, equilibrium equations (6) give $\sigma_{i 3,3}^{-1}=0$. Hence, by virtue of the boundary condition at $y_{3}=1 / 2$, one deduces that $\sigma_{i 3}^{-1}=0$ everywhere. Moreover, $\varepsilon_{\alpha \beta}^{-1}=0$ by virtue of (8) and $u_{\alpha}^{0}=0$. Then the constitutive relations (7) at order 0 give $\boldsymbol{\sigma}^{-1}=\mathbf{0}$ and $\boldsymbol{\varepsilon}^{-1}=\mathbf{0}$. The compatibility conditions (8) give now $U_{, \alpha}^{0}+u_{\alpha, 3}^{1}=0$ and $u_{3,3}^{1}=0$. After integrating these relations, since $u_{\alpha}^{1}=0$ at $y_{3}=0$, one finally obtains at this step

$$
\mathbf{u}^{1}\left(\overline{\mathbf{x}}, y_{3}\right)=-y_{3} U_{, \alpha}^{0}(\overline{\mathbf{x}}) \mathbf{e}_{\alpha}+U^{1}(\overline{\mathbf{x}}) \mathbf{e}_{3}, \quad \boldsymbol{\sigma}^{-1}=\mathbf{0}, \quad \varepsilon^{-1}=\mathbf{0},
$$

where the deflection at order $1, U^{1}(\overline{\mathbf{x}})$, has to be determined.

(3) Equilibrium equations, constitutive relations and compatibility conditions at order 0 . Because $\boldsymbol{\sigma}^{-1}=\mathbf{0}$, equilibrium equations (6) give $\sigma_{i 3,3}^{0}=0$. Hence, by virtue of the boundary condition at $y_{3}=1 / 2$, one deduces that $\sigma_{i 3}^{0}=0$ everywhere. Using (8) and (16) leads to $\varepsilon_{\alpha \beta}^{0}=-y_{3} U_{, \alpha \beta}^{0}$. Then the constitutive relations (7) at order 1 give $\boldsymbol{\sigma}^{0}$ and $\varepsilon^{0}$ which read as

$$
\left\{\begin{array}{l}
\sigma_{\alpha \beta}^{0}\left(\overline{\mathbf{x}}, y_{3}\right)=-\frac{y_{3}}{1-\nu^{2}}\left(\nu \Delta U^{0}(\overline{\mathbf{x}}) \delta_{\alpha \beta}+(1-\nu) U_{, \alpha \beta}^{0}(\overline{\mathbf{x}})\right), \quad \sigma_{\alpha 3}^{0}=\sigma_{33}^{0}=0, \\
\varepsilon_{\alpha \beta}^{0}\left(\overline{\mathbf{x}}, y_{3}\right)=-y_{3} U_{, \alpha \beta}^{0}(\overline{\mathbf{x}}), \quad \varepsilon_{\alpha 3}^{0}=0, \quad \varepsilon_{33}^{0}\left(\overline{\mathbf{x}}, y_{3}\right)=\frac{\nu y_{3}}{1-\nu} \Delta U^{0}(\overline{\mathbf{x}}) .
\end{array}\right.
$$

Inserting these relations into (8) and using the boundary condition $u_{\alpha}^{2}=0$ at $y_{3}=0$ leads to the following form for the displacements at order 2

$$
u_{\alpha}^{2}\left(\overline{\mathbf{x}}, y_{3}\right)=-y_{3} U_{, \alpha}^{1}(\overline{\mathbf{x}}), \quad u_{3}^{2}\left(\overline{\mathbf{x}}, y_{3}\right)=U^{2}(\overline{\mathbf{x}})+\frac{\nu y_{3}^{2}}{2(1-\nu)} \Delta U^{0}(\overline{\mathbf{x}})
$$

where the deflection at order $2, U^{2}(\overline{\mathbf{x}})$, has to be determined.

(4) Equilibrium equations, constitutive relations and compatibility conditions at order 1 . Using (17), equilibrium equations (6) give

$$
\sigma_{\alpha 3,3}^{1}=\frac{y_{3}}{1-\nu^{2}} \Delta U_{, \alpha}^{0}, \quad \sigma_{33,3}^{1}=0
$$

Hence, since $\sigma_{i 3}^{1}=0$ at $y_{3}=1 / 2$, one gets $\sigma_{i 3}^{1}$ everywhere. Moreover, since $\varepsilon_{\alpha \beta}^{1}$ is given by (18), the constitutive relations (7) allow us to obtain both $\boldsymbol{\sigma}^{1}$ and $\varepsilon^{1}$. Specifically $\boldsymbol{\sigma}^{1}$ reads

$$
\sigma_{\alpha \beta}^{1}\left(\overline{\mathbf{x}}, y_{3}\right)=-\frac{y_{3}}{1-\nu^{2}}\left(\nu \Delta U^{1}(\overline{\mathbf{x}}) \delta_{\alpha \beta}+(1-\nu) U_{, \alpha \beta}^{1}(\overline{\mathbf{x}})\right), \quad \sigma_{\alpha 3}^{1}\left(\overline{\mathbf{x}}, y_{3}\right)=\frac{4 y_{3}^{2}-1}{8\left(1-\nu^{2}\right)} \Delta U_{, \alpha}^{0}(\overline{\mathbf{x}}), \quad \sigma_{33}^{1}=0 .
$$

Remark (on the type of equations for $\left.\mathbf{u}^{n}\right)$. The first three relevant terms $\mathbf{u}^{n}(n=1,2,3)$ of the expansion of displacements allow for a simple mechanical interpretation. Indeed, equation (15) which identifies the components of the first term $\mathbf{u}^{0}$ descend from symmetry (material and of the loading) and the fact that (rescaled) normal stresses vanish on the surface. For $n=1$, relation (16) is a direct consequence of the fact that (rescaled) shear strains vanish alongside the associated stresses. On the other hand, for $n=2$, nonzero (rescaled) shear strains and stresses exist and are determined explicitly as a function of $y_{3}$ and the second derivative of the first nontrivial displacement $\mathbf{u}^{0}$, playing the role of a datum. Higher order terms $(n>2)$ exhibit a similar structure albeit with a datum that is a combination of higher derivatives of lower order modes. As a consequence, the datum entering in the equation of $\mathbf{u}^{n}$ for larger and larger $n$ is more and more singular. 


\subsubsection{First order plate equations}

1. First order shear forces and bending moments, plate equation for $U^{0}$. Inserting the previous results into (10) shows that shear forces and bending moments start at order $0: \mathcal{T}^{-3}=\mathcal{T}^{-2}=\mathcal{T}^{-1}=\mathbf{0}$ and $\mathcal{M}^{-2}=\mathcal{M}^{-1}=\mathbf{0}$. Moreover, inserting expression (17) of $\sigma_{\alpha \beta}^{0}$ into (10) gives the following constitutive relation between the bending moments at order 0 and the deflection at order 0

$$
\mathcal{M}_{\alpha \beta}^{0}(\overline{\mathbf{x}})=\mathrm{D}\left(\nu \Delta U^{0}(\overline{\mathbf{x}}) \delta_{\alpha \beta}+(1-\nu) U_{, \alpha \beta}^{0}(\overline{\mathbf{x}})\right) \quad \text { with } \quad \mathrm{D}=\frac{1}{12\left(1-\nu^{2}\right)} .
$$

Then, using (11) at order 0 gives $\mathcal{T}^{0}$ in terms of $U^{0}: \mathcal{T}_{\alpha}^{0}(\overline{\mathbf{x}})=-\mathrm{D} \Delta U_{, \alpha}^{0}(\overline{\mathbf{x}})$. Inserting (20) into (13) taken at order 0 leads to the well-known bilaplacian equation which governs the plate deflection. In terms of dimensionless quantities it reads here

$$
\mathrm{D} \Delta^{2} U^{0}-\mathcal{F}^{0}=0 \quad \text { in } \quad \Omega_{0}
$$

Note that $U^{0}$ is not (everywhere) zero as soon as $\mathcal{F}^{0}$ is not zero, that justifies a posteriori our choice (5) of the order of magnitude of the external forces. To complete this fourth order partial differential equation one must give the boundary condition satisfied by $U^{0}$ on the sides of $\partial \Omega_{0}$. That will be achieved in the next section where are studied the boundary layers.

2. Shear forces and bending moments at order 1 , plate equation for $U^{1}$. Inserting the expression (19) of $\sigma_{\alpha \beta}^{1}$ into (10) gives the constitutive relation between the bending moments and the deflection at order 1 :

$$
\mathcal{M}_{\alpha \beta}^{1}(\overline{\mathbf{x}})=\mathrm{D}\left(\nu \Delta U^{1}(\overline{\mathbf{x}}) \delta_{\alpha \beta}+(1-\nu) U_{, \alpha \beta}^{1}(\overline{\mathbf{x}})\right)
$$

which is similar to (20). Then, using (11) at order 1 gives $\mathcal{T}^{1}$ in terms of $U^{1}: \mathcal{T}_{\alpha}^{1}(\overline{\mathbf{x}})=-\mathrm{D} \Delta U_{, \alpha}^{1}(\overline{\mathbf{x}})$. Inserting (22) into (13) taken at order 1 leads to the bilaplacian equation for the plate deflection $U^{1}$

$$
\mathrm{D} \Delta^{2} U^{1}=0 \quad \text { in } \quad \Omega_{0},
$$

to which one must add two boundary conditions on each side. Let us note that $U^{1}$ like $U^{0}$ are governed by the bilaplacian operator in the bulk. The only difference will come from the boundary conditions. A change shall appear for $U^{2}$, governed by a different fourth order partial differential equation in the bulk, in conformity with the Mindlin-Reissner theory. Accordingly, boundary layers effects appear at an order lower than those due to a weakening of the kinematic constraint on the rotation.

It remains to determine the boundary conditions that the fields $U^{0}$ and $U^{1}$ have to satisfy. These boundary conditions must be deduced from the true three dimensional boundary conditions. If one considers the expansion of the displacements just obtained, one sees from (15)-(16) that $\mathbf{u}^{0}=\mathbf{u}^{1}=\mathbf{0}$ at $\bar{x}_{1}= \pm 1 / 2$ provided that $U^{0}=U^{1}=U_{, 1}^{0}=0$ at $\bar{x}_{1}= \pm 1 / 2$. But, when $\nu \neq 0$, (18) shows that $\mathbf{u}^{2}=0$ only if $\Delta U^{0}=0$ at $\bar{x}_{1}= \pm 1 / 2$, which is in general impossible. That means that $\mathbf{u}^{2}$ cannot in general satisfy the right boundary conditions and hence that the previous expansion is not valid in the neighbourhood of $\bar{x}_{1}= \pm 1 / 2$. A boundary layer is present. In the same manner, if we consider the expansion of stresses that has been obtained, one sees from (17) that, at $\bar{x}_{2}= \pm 1 / 2, \boldsymbol{\sigma}^{0} \mathbf{e}_{2}=\mathbf{0}$ provided that $U_{, 12}^{0}=\nu U_{, 11}^{0}+U_{, 22}^{0}=0$ and from (19) that $\boldsymbol{\sigma}^{1} \mathbf{e}_{2}=\mathbf{0}$ provided that $U_{, 12}^{1}=\nu U_{, 11}^{1}+U_{, 22}^{1}=\Delta U_{, 2}^{0}=0$. But this latter equality does not hold in general. Hence the proposed expansion is not valid in the neighbourhood of $\bar{x}_{2}= \pm 1 / 2$ and a boundary layer is also present. The two next sections are devoted to the analysis of these boundary layers.

\section{Analysis of the boundary layers near the clamped sides of the plate}

\subsection{Notations}

We will only develop the analysis of the boundary layer near the side $\bar{x}_{1}=-1 / 2$, the study of the other side $\bar{x}_{1}=+1 / 2$ being essentially the same. Throughout this subsection we use the following notations: we set

$$
y_{1}:=\frac{2 \bar{x}_{1}+1}{2 \eta}
$$

and hence the pair $\left(y_{1}, y_{3}\right)$ represents the small scale coordinates whereas $\bar{x}_{2}$ is the large scale coordinate. Specifically, the change of variable above maps the vicinity of the boundary into a strip of length $1 / \eta$, unbounded from above when $\eta \ll 1$. Consequently, boundary layer problems will be posed on the semi-infinite strip $\mathrm{Y}=(0,+\infty) \times(0,+1 / 2)$ of the $\left(y_{1}, y_{3}\right)$ plane, whereas $\bar{x}_{2}$ will only play the role of a parameter varying in $I_{2}=(-\bar{\ell} / 2, \bar{\ell} / 2)$. Accordingly, we denote by $\mathbf{y}$ the pair $\left(y_{1}, y_{3}\right)$ in $\mathrm{Y}$. When $\mathbf{v}=\mathbf{v}\left(\bar{x}_{2}, \mathbf{y}\right)$ is a vector field defined in 
$I_{2} \times \mathrm{Y}$, we denote by $\varepsilon_{\mathbf{y}}(\mathbf{v})$ and $\varepsilon_{\bar{x}}(\mathbf{v})$ the tensor fields whose matrix representations in the basis $\left(\mathbf{e}_{1}, \mathbf{e}_{2}, \mathbf{e}_{3}\right)$ are the following

$$
\boldsymbol{\varepsilon}_{\mathbf{y}}(\mathbf{v})=\left(\begin{array}{ccc}
\frac{\partial v_{1}}{\partial y_{1}} & \frac{1}{2} \frac{\partial v_{2}}{\partial y_{1}} & \frac{1}{2}\left(\frac{\partial v_{3}}{\partial y_{1}}+\frac{\partial v_{1}}{\partial y_{3}}\right) \\
\frac{1}{2} \frac{\partial v_{2}}{\partial y_{1}} & 0 & \frac{1}{2} \frac{\partial v_{2}}{\partial y_{3}} \\
\frac{1}{2}\left(\frac{\partial v_{3}}{\partial y_{1}}+\frac{\partial v_{1}}{\partial y_{3}}\right) & \frac{1}{2} \frac{\partial v_{2}}{\partial y_{3}} & \frac{\partial v_{3}}{\partial y_{3}}
\end{array}\right), \quad \boldsymbol{\varepsilon}_{\bar{x}}(\mathbf{v})=\left(\begin{array}{ccc}
0 & \frac{1}{2} \frac{\partial v_{1}}{\partial \bar{x}_{2}} & 0 \\
\frac{1}{2} \frac{\partial v_{1}}{\partial \bar{x}_{2}} & \frac{\partial v_{2}}{\partial \bar{x}_{2}} & \frac{1}{2} \frac{\partial v_{3}}{\partial \bar{x}_{2}} \\
0 & \frac{1}{2} \frac{\partial v_{3}}{\partial \bar{x}_{2}} & 0
\end{array}\right) .
$$

When $\boldsymbol{\tau}=\boldsymbol{\tau}\left(\bar{x}_{2}, \mathbf{y}\right)$ is a symmetric second order tensor field defined in $I_{2} \times \mathrm{Y}$, we denote by $\operatorname{div}_{\mathbf{y}} \boldsymbol{\tau}$ and $\operatorname{div}_{\bar{x}} \boldsymbol{\tau}$ the vectors fields whose representations in the basis $\left(\mathbf{e}_{1}, \mathbf{e}_{2}, \mathbf{e}_{3}\right)$ are

$$
\operatorname{div}_{\mathbf{y}} \boldsymbol{\tau}=\left(\frac{\partial \tau_{i 1}}{\partial y_{1}}+\frac{\partial \tau_{i 3}}{\partial y_{3}}\right) \mathbf{e}_{i}, \quad \operatorname{div}_{\bar{x}} \boldsymbol{\tau}=\frac{\partial \tau_{i 2}}{\partial \bar{x}_{2}} \mathbf{e}_{i} .
$$

The following Lemma, characterising a displacement field $\mathbf{v}$ whose associated strain field $\varepsilon_{\mathbf{y}}(\mathbf{v})$ vanishes, is of constant use (its proof is left to the reader).

Lemma 1 If a vector field $\mathbf{v}$ is such that $\boldsymbol{\varepsilon}_{\mathbf{y}}(\mathbf{v})=0$ in $I_{2} \times \mathrm{Y}$ (in the sense of (23)), then $\mathbf{v}$ is necessarily of the form

$$
\mathbf{v}\left(\bar{x}_{2}, \mathbf{y}\right)=\mathbf{V}\left(\bar{x}_{2}\right)+\omega\left(\bar{x}_{2}\right) \mathbf{e}_{2} \wedge \mathbf{y} .
$$

where $\wedge$ denotes the vector cross-product. Furthermore, for such a $\mathbf{v}$, one has

(i) if $v_{1}=v_{2}=0$ on $y_{3}=0$ then $\mathbf{v}$ is of the form $\mathbf{v}\left(\bar{x}_{2}, \mathbf{y}\right)=V\left(\bar{x}_{2}\right) \mathbf{e}_{3}+\omega\left(\bar{x}_{2}\right) \mathbf{e}_{2} \wedge \mathbf{y}$;

(ii) if $\mathbf{v}=\mathbf{0}$ on $y_{1}=0$ then $\mathbf{v}=\mathbf{0}$ in $I_{2} \times \mathrm{Y}$.

3.2 Hypothesis on the form of the expansions and statement of the matching conditions

We suppose that, near the side $\bar{x}_{1}=-1 / 2$ of the plate, the displacement $\mathbf{u}^{\eta}$ admits the following asymptotic expansion with respect to the small parameter $\eta$

$$
\mathbf{u}^{\eta}\left(\overline{\mathbf{x}}, y_{3}\right)=\mathbf{v}^{0}\left(\bar{x}_{2}, \mathbf{y}\right)+\eta \mathbf{v}^{1}\left(\bar{x}_{2}, \mathbf{y}\right)+\eta^{2} \mathbf{v}^{2}\left(\bar{x}_{2}, \mathbf{y}\right)+\eta^{3} \mathbf{v}^{3}\left(\bar{x}_{2}, \mathbf{y}\right)+\cdots,
$$

with $\bar{x}_{2} \in I_{2}, \mathbf{y}=\left(y_{1}, y_{3}\right) \in \mathrm{Y}$ and $\eta y_{1}=\bar{x}_{1}+1 / 2$. In other words, we introduce the small scale variable $y_{1}$ instead of $\bar{x}_{1}$. Consequently, the strains and stresses admit the same type of asymptotic expansion which a priori starts at order -2

$$
\left\{\begin{array}{l}
\varepsilon^{\eta}\left(\overline{\mathbf{x}}, y_{3}\right)=\eta^{-2} \boldsymbol{\gamma}^{-2}\left(\bar{x}_{2}, \mathbf{y}\right)+\eta^{-1} \boldsymbol{\gamma}^{-1}\left(\bar{x}_{2}, \mathbf{y}\right)+\boldsymbol{\gamma}^{0}\left(\bar{x}_{2}, \mathbf{y}\right)+\eta \boldsymbol{\gamma}^{1}\left(\bar{x}_{2}, \mathbf{y}\right)+\eta^{2} \boldsymbol{\gamma}^{2}\left(\bar{x}_{2}, \mathbf{y}\right)+\cdots \\
\boldsymbol{\sigma}^{\eta}\left(\overline{\mathbf{x}}, y_{3}\right)=\eta^{-2} \boldsymbol{\tau}^{-2}\left(\bar{x}_{2}, \mathbf{y}\right)+\eta^{-1} \boldsymbol{\tau}^{-1}\left(\bar{x}_{2}, \mathbf{y}\right)+\boldsymbol{\tau}^{0}\left(\bar{x}_{2}, \mathbf{y}\right)+\eta \boldsymbol{\tau}^{1}\left(\bar{x}_{2}, \mathbf{y}\right)+\eta^{2} \boldsymbol{\tau}^{2}\left(\bar{x}_{2}, \mathbf{y}\right)+\cdots
\end{array}\right.
$$

These expansions must match those introduced in the previous section. Matching conditions are obtained by requiring that the two expansions are valid in an intermediate zone where $\bar{x}_{1}+1 / 2$ is small while $y_{1}$ is large. Specifically, starting from (14) leads to

$$
\mathbf{u}^{\eta}\left(\overline{\mathbf{x}}, y_{3}\right)=\sum_{i \in \mathbb{N}} \eta^{i} \mathbf{u}^{i}\left(-1 / 2+\eta y_{1}, \bar{x}_{2}, y_{3}\right) .
$$

Then, assuming that the $\mathbf{u}^{i}$ 's can be expanded with respect to $\bar{x}_{1}$ near $\bar{x}_{1}=-1 / 2$, we write

$$
\mathbf{u}^{i}\left(-1 / 2+\eta y_{1}, \bar{x}_{2}, y_{3}\right)=\mathbf{u}^{i}\left(-1 / 2, \bar{x}_{2}, y_{3}\right)+\eta y_{1} \mathbf{u}_{, 1}^{i}\left(-1 / 2, \bar{x}_{2}, y_{3}\right)+\frac{1}{2} \eta^{2} y_{1}^{2} \mathbf{u}_{, 11}^{i}\left(-1 / 2, \bar{x}_{2}, y_{3}\right)+\cdots .
$$

Inserting the above expansion into (26) and comparing with (25) gives the desired matching conditions. We will only use the first three which read

$$
\left\{\begin{array}{l}
\mathbf{u}^{0}\left(-\frac{1}{2}, \bar{x}_{2}, y_{3}\right)=\lim _{y_{1} \rightarrow \infty} \mathbf{v}^{0}\left(\bar{x}_{2}, \mathbf{y}\right) \\
\mathbf{u}^{1}\left(-\frac{1}{2}, \bar{x}_{2}, y_{3}\right)=\lim _{y_{1} \rightarrow \infty}\left(\mathbf{v}^{1}\left(\bar{x}_{2}, \mathbf{y}\right)-y_{1} \mathbf{u}_{1}^{0}\left(-\frac{1}{2}, \bar{x}_{2}, y_{3}\right)\right) \\
\mathbf{u}^{2}\left(-\frac{1}{2}, \bar{x}_{2}, y_{3}\right)=\lim _{y_{1} \rightarrow \infty}\left(\mathbf{v}^{2}\left(\bar{x}_{2}, \mathbf{y}\right)-y_{1} \mathbf{u}_{1,1}^{1}\left(-\frac{1}{2}, \bar{x}_{2}, y_{3}\right)-\frac{1}{2} y_{1}^{2} \mathbf{u}_{, 11}^{0}\left(-\frac{1}{2}, \bar{x}_{2}, y_{3}\right)\right)
\end{array}\right.
$$

The same type of matching conditions hold for stresses, except that they start at order -2 instead of 0 (and hence all the indices must be shifted). 
3.3 Obtention of three kinematic boundary conditions from the determination of $\mathbf{v}^{0}$ and $\mathbf{v}^{1}$

Inserting the assumed expansions into (6)-(9) gives for the leading terms the following set of equations

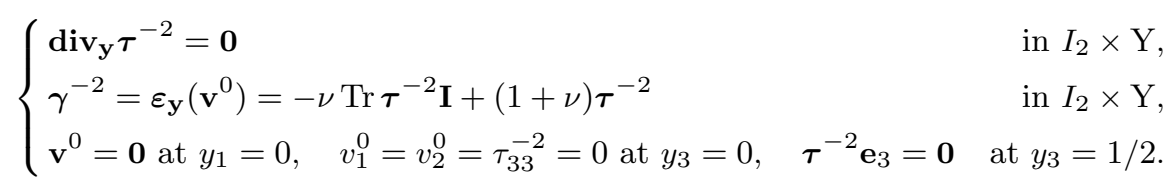

Moreover, since $\boldsymbol{\sigma}^{-2}=\mathbf{0}$, the first matching condition for stresses gives $\boldsymbol{\tau}^{-2}\left(\bar{x}_{2},+\infty, y_{3}\right)=0$. Multiplying the equilibrium equation by $\mathbf{v}^{0}$ and integrating over $I_{2} \times \mathrm{Y}$ leads to

$$
\int_{I_{2} \times \mathrm{Y}} \operatorname{div}_{\mathbf{y}} \boldsymbol{\tau}^{-2} \cdot \mathbf{v}^{0} d \bar{x}_{2} d \mathbf{y}=0
$$

Integrating by parts the integral, using boundary conditions, matching conditions, and (23)-(24) gives

$$
\int_{I_{2} \times \mathrm{Y}} \boldsymbol{\tau}^{-2} \cdot \varepsilon_{\mathbf{y}}\left(\mathbf{v}^{0}\right) d \bar{x}_{2} d \mathbf{y}=0
$$

Then, by the constitutive relations between $\boldsymbol{\tau}^{-2}$ and $\varepsilon_{\mathbf{y}}\left(\mathbf{v}^{0}\right)$, one can conclude that $\boldsymbol{\varepsilon}_{\mathbf{y}}\left(\mathbf{v}^{0}\right)=\mathbf{0}$. Finally, Lemma 1 gives

$$
\mathbf{v}^{0}=\mathbf{0}, \quad \boldsymbol{\tau}^{-2}=\mathbf{0}, \quad \boldsymbol{\gamma}^{-2}=\mathbf{0} \quad \text { in } I_{2} \times \mathrm{Y}
$$

Since $\mathbf{v}^{0}=\mathbf{0}$, the first matching condition (27) combined with (15) gives the expected boundary condition that $U^{0}$ must satisfy on the side $\bar{x}_{1}=-1 / 2$

$$
U^{0}=0 \quad \text { at } \quad \bar{x}_{1}=-1 / 2 \text {. }
$$

Considering now the next terms of the expansion, one deduces from (6)-(9) that $\boldsymbol{\tau}^{-1}$ and $\mathbf{v}^{1}$ must satisfy reinsert label here

$$
\left\{\begin{array}{lr}
\operatorname{div}_{\mathbf{y}} \boldsymbol{\tau}^{-1}=\mathbf{0} & \text { in } I_{2} \times \mathrm{Y} \\
\boldsymbol{\varepsilon}_{\mathbf{y}}\left(\mathbf{v}^{1}\right)=-\nu \operatorname{Tr} \boldsymbol{\tau}^{-1} \mathbf{I}+(1+\nu) \boldsymbol{\tau}^{-1} & \text { in } I_{2} \times \mathrm{Y} \\
\mathbf{v}^{1}=\mathbf{0} \text { at } y_{1}=0, \quad v_{1}^{1}=v_{2}^{1}=\tau_{33}^{-1}=0 \text { at } y_{3}=0, \quad \boldsymbol{\tau}^{-1} \mathbf{e}_{3}=\mathbf{0} & \text { at } y_{3}=1 / 2
\end{array}\right.
$$

Because $\boldsymbol{\sigma}^{-1}=\mathbf{0}$, one can follow the same procedure as for $\mathbf{v}^{0}$ and $\boldsymbol{\tau}^{-2}$ to finally obtain

$$
\mathbf{v}^{1}=\mathbf{0}, \quad \boldsymbol{\tau}^{-1}=\mathbf{0}, \quad \gamma^{-1}=\mathbf{0} \quad \text { in } \quad I_{2} \times \mathrm{Y}
$$

Using (15), (16), (28) and (29), the second matching condition (27) reads

$$
-y_{3} U_{, 1}^{0}\left(-1 / 2, \bar{x}_{2}\right) \mathbf{e}_{1}+U^{1}\left(-1 / 2, \bar{x}_{2}\right) \mathbf{e}_{3}=\lim _{y_{1} \rightarrow+\infty}\left(-y_{1} U_{, 1}^{0}\left(-1 / 2, \bar{x}_{2}\right) \mathbf{e}_{3}\right),
$$

and hence is satisfied if and only if $U^{0}$ and $U^{1}$ verify the following boundary conditions on the side $\bar{x}_{1}=-1 / 2$

$$
U_{, 1}^{0}=0, \quad U^{1}=0 \quad \text { at } \quad \bar{x}_{1}=-1 / 2 .
$$

Therefore we have obtained the two required boundary conditions that $U^{0}$ must satisfy on the clamped sides (one can follow the same procedure to obtain the same conditions at $\left.\bar{x}_{1}=+1 / 2\right)$ and these relations are the usual ones of fixed deflection and rotation. At this stage, we have only obtained the condition of fixed deflection for $U^{1}$, the condition on its rotation will be obtained in the next subsections after determining $\mathbf{v}^{2}$. 
3.4 Determination of $\mathbf{v}^{2}$

Using the previous results, one deduces from (6)-(9) that $\tau^{0}$ and $\mathbf{v}^{2}$ must satisfy

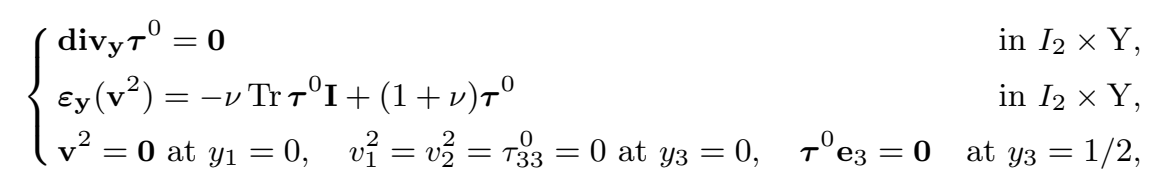

but, because in general $\boldsymbol{\sigma}^{0} \neq \mathbf{0}$ by virtue of (17), one cannot conclude as before. Indeed, the matching condition between $\sigma^{0}$ and $\tau^{0}$ reads

$$
\lim _{y_{1} \rightarrow+\infty} \boldsymbol{\tau}^{0}\left(\bar{x}_{2}, \mathbf{y}\right)=\boldsymbol{\sigma}^{0}\left(-\frac{1}{2}, \bar{x}_{2}, y_{3}\right)=-\frac{y_{3}}{1-\nu^{2}} U_{, 11}^{0}\left(-\frac{1}{2}, \bar{x}_{2}\right)\left(\mathbf{e}_{1} \otimes \mathbf{e}_{1}+\nu \mathbf{e}_{2} \otimes \mathbf{e}_{2}\right),
$$

where we have used $(17)$ and $U_{, 12}^{0}=U_{, 22}^{0}=0$ at $\bar{x}_{1}=-1 / 2$. Therefore, when $U_{, 11}^{0}\left(-1 / 2, \bar{x}_{2}\right)$ is given, the system (31) with (32) is a linear problem for $\mathbf{v}^{2}$ posed on $I_{2} \times \mathrm{Y}$.

By linearity, this problem can be decomposed into two problems: an "out-of-plane problem" for $v_{2}^{2}$ and an "in-plane problem" for $\left(v_{1}^{2}, v_{3}^{2}\right)$. First, using (23) and (24), one deduces from (31) and (32) that $v_{2}^{2}$ is given by

$$
\left\{\begin{array}{l}
\Delta_{\mathbf{y}} v_{2}^{2}=0 \quad \text { in } \quad I_{2} \times \mathrm{Y}, \\
v_{2}^{2}=0 \text { at } y_{1}=0 \text { and at } y_{3}=0, \quad \frac{\partial v_{2}^{2}}{\partial y_{3}}=0 \text { at } y_{3}=1 / 2, \quad \frac{\partial v_{2}^{2}}{\partial y_{1}}=0 \text { at } y_{1}=+\infty
\end{array}\right.
$$

One easily checks that $v_{2}^{2}=0$ is the unique solution and hence that the problem for $\mathbf{v}^{2}$ is reduced to a plane strain problem. Using the linearity, $\mathbf{v}^{2}$ and $\boldsymbol{\tau}^{0}$ can be read as

$$
\mathbf{v}^{2}\left(\bar{x}_{2}, \mathbf{y}\right)=U_{, 11}^{0}\left(-\frac{1}{2}, \bar{x}_{2}\right)\left(\mathbf{V}^{*}(\mathbf{y})+\mathbf{V}^{\mathbf{c}}(\mathbf{y})\right), \quad \boldsymbol{\tau}^{0}\left(\bar{x}_{2}, \mathbf{y}\right)=U_{, 11}^{0}\left(-\frac{1}{2}, \bar{x}_{2}\right)\left(\mathbf{\Sigma}^{*}(\mathbf{y})+\mathbf{\Sigma}^{\mathbf{c}}(\mathbf{y})\right)
$$

where $\mathbf{V}^{*}$ and $\boldsymbol{\Sigma}^{*}$ are the following displacement and stress tensor fields defined in $\mathrm{Y}$ :

$$
\mathbf{V}^{*}(\mathbf{y})=-y_{1} y_{3} \mathbf{e}_{1}+\left(\frac{y_{1}^{2}}{2}+\frac{\nu y_{3}^{2}}{2(1-\nu)}\right) \mathbf{e}_{3}, \quad \mathbf{\Sigma}^{*}(\mathbf{y})=-\frac{y_{3}}{1-\nu^{2}}\left(\mathbf{e}_{1} \otimes \mathbf{e}_{1}+\nu \mathbf{e}_{2} \otimes \mathbf{e}_{2}\right)
$$

In (33) the displacement field $\mathbf{V}^{\mathbf{c}}$ and the stress field $\mathbf{\Sigma}^{\mathbf{c}}$ have the following form

$$
\mathbf{V}^{\mathbf{c}}=\left(\begin{array}{c}
V_{1}^{c} \\
0 \\
V_{3}^{c}
\end{array}\right), \quad \mathbf{\Sigma}^{\mathbf{c}}=\left(\begin{array}{ccc}
\Sigma_{11}^{c} & 0 & \Sigma_{13}^{c} \\
0 & \nu\left(\Sigma_{11}^{c}+\Sigma_{33}^{c}\right) & 0 \\
\Sigma_{13}^{c} & 0 & \Sigma_{33}^{c}
\end{array}\right)
$$

and are solution of the following plane strain problem posed in Y, called the bending boundary layer problem at a clamped side:

$$
\left\{\begin{array}{lr}
\operatorname{div}_{\mathbf{y}} \boldsymbol{\Sigma}^{\mathbf{c}}=\mathbf{0} & \text { in Y, } \\
\boldsymbol{\varepsilon}_{\mathbf{y}}\left(\mathbf{V}^{\mathbf{c}}\right)=-\nu \operatorname{Tr} \mathbf{\Sigma}^{\mathbf{c}} \mathbf{I}+(1+\nu) \mathbf{\Sigma}^{\mathbf{c}} & \text { in Y, } \\
\mathbf{V}^{\mathbf{c}}=-\frac{\nu y_{3}^{2}}{2(1-\nu)} \mathbf{e}_{3} & \text { at } y_{1}=0, \\
V_{1}^{c}=\Sigma_{33}^{c}=0 \text { at } y_{3}=0, \quad \mathbf{\Sigma}^{\mathbf{c}} \mathbf{e}_{3}=\mathbf{0} & \text { at } y_{3}=1 / 2, \\
\lim _{y_{1} \rightarrow+\infty} \boldsymbol{\Sigma}^{\mathbf{c}}(\mathbf{y})=\mathbf{0} . &
\end{array}\right.
$$

To obtain (35), one in particular uses $\operatorname{div}_{\mathbf{y}} \boldsymbol{\Sigma}^{*}=\mathbf{0}$ and $\varepsilon_{\mathbf{y}}\left(\mathbf{V}^{*}\right)=-\nu \operatorname{Tr} \boldsymbol{\Sigma}^{*} \mathbf{I}+(1+\nu) \boldsymbol{\Sigma}^{*}$. The study of this problem is made in the next subsection and will give the second boundary condition for $U^{1}$ (and a first one for $U^{2}$ ). 


\subsection{Obtention of the second boundary condition for $U^{1}$ and a first one for $U^{2}$}

By classical arguments one can prove that the bending boundary layer problem (35) admits a unique solution. Let us first remark that this solution depends only on the Poisson ratio $\nu$ and hence can be considered as universal for a homogeneous plate near a clamped boundary. (However, one should prove that if one considered a curved, instead of a straight boundary, the problem would not change.) The solution is $\mathbf{V}^{\mathbf{c}}=\mathbf{0}$ and $\boldsymbol{\Sigma}^{\mathbf{c}}=\mathbf{0}$ if and only if $\nu=0$ by virtue of the boundary condition on $y_{1}=0$. When $\nu \neq 0$, a boundary layer appears because $\mathbf{u}^{2}$ cannot satisfy the clamped boundary conditions. Because $\boldsymbol{\Sigma}^{\mathbf{c}}$ tends to $\mathbf{0}$ when $y_{1}$ tends to infinity, so does $\boldsymbol{\varepsilon}_{\mathbf{y}}\left(\mathbf{V}^{\mathbf{c}}\right)$. Consequently, since $V_{1}^{c}=0$ on $y_{1}=0$, by virtue of Lemma 1 , there exists two constants $\mathrm{K}_{\mathrm{c}}$ and $\mathrm{C}_{\mathrm{c}}$ (depending only on $\nu$ ) such that

$$
\lim _{y_{1} \rightarrow+\infty}\left(\mathbf{V}^{\mathbf{c}}(\mathbf{y})-\mathrm{C}_{\mathrm{c}} \mathbf{e}_{2} \wedge \mathbf{y}\right)=\mathrm{K}_{\mathrm{c}} \mathbf{e}_{3} .
$$

In fact, it can be proved that the decay of $\mathbf{V}^{\mathbf{c}}$ to a rigid body motion is exponential with respect to $y_{1}$, but this proof is outside the scope of the present paper and we simply check it numerically (the computations are made with a finite element code). One can see the rapid decay in Figure 1 where the graphs of $y_{1} \mapsto V_{1}^{c}\left(y_{1}, y_{3}\right)$ and of $y_{1} \mapsto V_{3}^{c}\left(y_{1}, y_{3}\right)$ are plotted for several values of $y_{3}$ when $\nu=1 / 4$. The asymptotic relation above provides the mechanical interpretation of the coefficients $\mathrm{C}_{c}$ and $\mathrm{K}_{c}$ which can be respectively regarded as the limit values of displacement and rotation associated to the outer solution, on $y_{1}=0$.
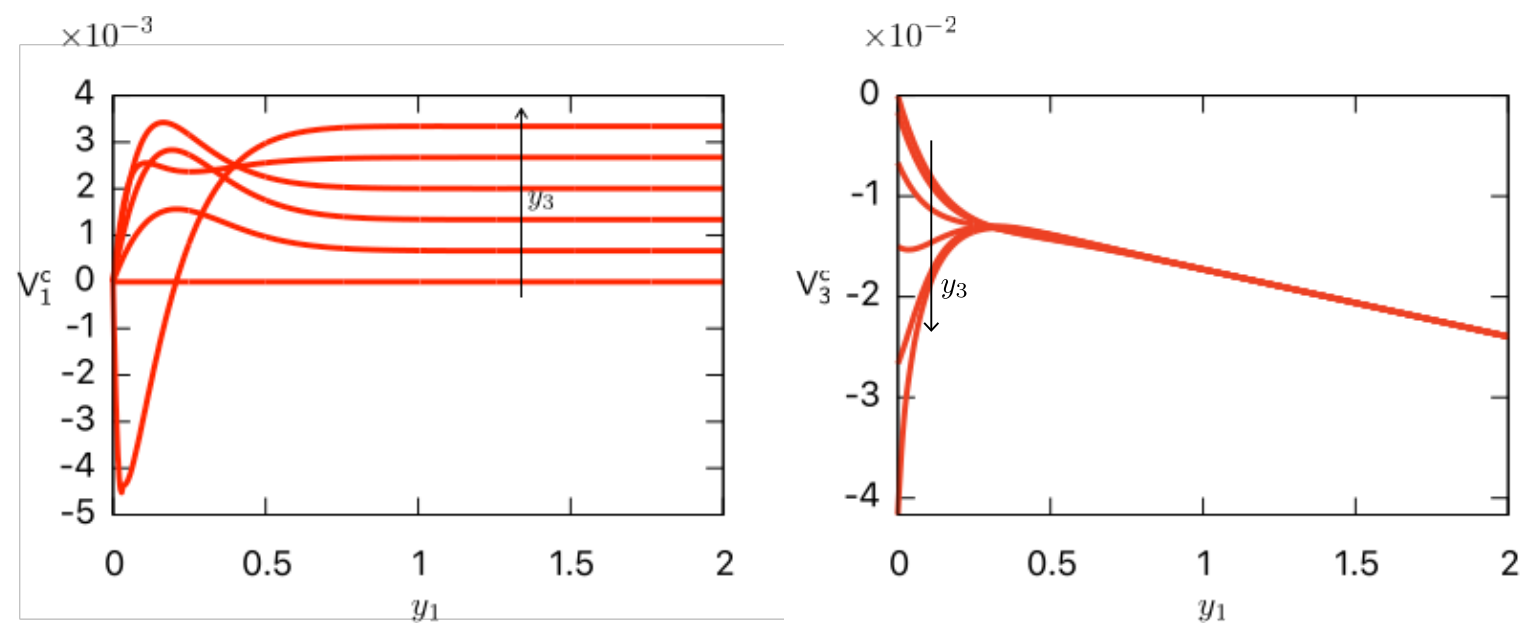

Fig. 1 Graph of $V_{1}^{c}$ (left) and $V_{3}^{c}$ (right) as a function of $y_{1}$ for six values of $y_{3}, y_{3}=0 ., 0.1,0.2,0.3,0.4,0.5$, when $\nu=0.25$. It can be seen that $V_{1}^{c}$ tends rapidly, when $y_{1}$ grows, to a constant which is proportional to $y_{3}$ whereas $V_{3}^{c}$ tends to an affine function independent of $y_{3}$. The slope of this asymptotic line is $-\mathrm{C}_{\mathrm{c}}$ and its intersection with the axis $y_{1}=0$ is $\mathrm{K}_{\mathrm{c}}$ which represent, respectively, the trace at the boundary of the limit rotation and that of the limit displacement associated to the macroscopic (bulk) solution.

Accordingly, using (15), (16), (18), (30) and (33), the third matching condition (27) becomes

$$
\left\{\begin{array}{l}
-y_{3} U_{, 1}^{1}\left(-\frac{1}{2}, \bar{x}_{2}, y_{3}\right)=\lim _{y_{1} \rightarrow \infty} V_{1}^{c}\left(\bar{x}_{2}, \mathbf{y}\right) U_{, 11}^{0}\left(-\frac{1}{2}, \bar{x}_{2}, y_{3}\right) \\
U^{2}\left(-\frac{1}{2}, \bar{x}_{2}, y_{3}\right)=\lim _{y_{1} \rightarrow \infty}\left(V_{3}^{c}\left(\bar{x}_{2}, \mathbf{y}\right) U_{, 11}^{0}\left(-\frac{1}{2}, \bar{x}_{2}, y_{3}\right)-y_{1} U_{, 1}^{1}\left(-\frac{1}{2}, \bar{x}_{2}, y_{3}\right)\right) .
\end{array}\right.
$$

Then, by virtue of (36), one gets

$$
U_{, 1}^{1}=-\mathrm{C}_{\mathrm{c}} U_{, 11}^{0}, \quad U^{2}=\mathrm{K}_{\mathrm{c}} U_{, 11}^{0} \quad \text { at } \quad \bar{x}_{1}=-1 / 2 .
$$

The first relation in (37) says that the (normal) rotation satisfied by $U^{1}$ on a clamped side is proportional to the normal bending moment $\mathcal{M}_{11}^{0}$. Indeed, using (20), one gets $\mathrm{D} U_{, 1}^{1}=-\mathrm{C}_{\mathrm{c}} \mathcal{M}_{11}^{0}$ at $\bar{x}_{1}=-1 / 2$. Consequently, one has obtained a boundary condition of Robin's type where the rotation is proportional to the bending moment and the constant $C_{c}$ gives the compliance of the angular spring. Let us show that this coefficient which depends only on $\nu$ is always non negative (vanishing only when $\nu=0$ ) by giving an energetic interpretation of it. Specifically, the following relation holds true:

$$
\mathrm{C}_{\mathrm{c}}=24\left(1-\nu^{2}\right) \int_{\mathrm{Y}} \mathbf{\Sigma}^{\mathbf{c}}(\mathbf{y}) \cdot \varepsilon_{\mathbf{y}}\left(\mathbf{V}^{\mathbf{c}}\right)(\mathbf{y}) d \mathbf{y} \geq 0
$$


which means that $C_{c}$ is given by the elastic energy associated with the boundary layer displacement corrector $\mathbf{V}^{\mathbf{c}}$. To prove (38), let us first start from the equilibrium equation $\operatorname{div}_{\mathbf{y}} \boldsymbol{\Sigma}^{\mathbf{c}}=\mathbf{0}$ that one multiplies by $\mathbf{V}^{\mathbf{c}}+\mathbf{V}^{*}$ and integrates over $\mathrm{Y}$ to get

$$
\int_{\mathbf{Y}} \operatorname{div}_{\mathbf{y}} \boldsymbol{\Sigma}^{\mathbf{c}} \cdot\left(\mathbf{V}^{\mathbf{c}}+\mathbf{V}^{*}\right) d \mathbf{y}=0
$$

Integrating by parts the integral leads to

$$
\int_{\mathbf{Y}} \mathbf{\Sigma}^{\mathbf{c}} \cdot \varepsilon_{\mathbf{y}}\left(\mathbf{V}^{\mathbf{c}}+\mathbf{V}^{*}\right) d \mathbf{y}=0
$$

because all boundary terms vanish by virtue of the exponential decay of $\boldsymbol{\Sigma}^{\mathbf{c}}$ when $y_{1}$ goes to infinity and the boundary conditions satisfied by $\boldsymbol{\Sigma}^{\mathbf{c}}, \mathbf{V}^{\mathbf{c}}$ and $\mathbf{V}^{*}$. Let us now remark that $\boldsymbol{\Sigma}^{\mathbf{c}} \cdot \varepsilon_{\mathbf{y}}\left(\mathbf{V}^{*}\right)=\boldsymbol{\Sigma}^{*} \cdot \varepsilon_{\mathbf{y}}\left(\mathbf{V}^{\mathbf{c}}\right)$ by virtue of the constitutive relations between $\boldsymbol{\Sigma}^{\mathbf{c}}$ and $\varepsilon_{\mathbf{y}}\left(\mathbf{V}^{\mathbf{c}}\right)$, or $\boldsymbol{\Sigma}^{*}$ and $\boldsymbol{\varepsilon}_{\mathbf{y}}\left(\mathbf{V}^{*}\right)$. Accordingly, using the expression (34) of $\boldsymbol{\Sigma}^{\mathbf{c}}$ and the properties of $\mathbf{V}^{\mathbf{c}}$ at $y_{1}=0$ and at $y_{1}=+\infty$ allows us to obtain

$$
\int_{\mathrm{Y}} \boldsymbol{\Sigma}^{\mathbf{c}} \cdot \boldsymbol{\varepsilon}_{\mathbf{y}}\left(\mathbf{V}^{\mathbf{c}}\right) d \mathbf{y}=-\int_{\mathrm{Y}} \boldsymbol{\Sigma}^{*} \cdot \varepsilon_{\mathbf{y}}\left(\mathbf{V}^{\mathbf{c}}\right) d \mathbf{y}=\int_{\mathrm{Y}} \frac{y_{3}}{1-\nu^{2}} V_{1,1}^{c} d \mathbf{y}=\int_{0}^{1 / 2} \frac{\mathrm{C}_{\mathrm{c}} y_{3}^{2}}{1-\nu^{2}} d y_{3}=\frac{\mathrm{C}_{\mathrm{c}}}{24\left(1-\nu^{2}\right)}
$$

that is the desired result. Such an interpretation does not exist for $\mathrm{K}_{\mathrm{c}}$ and, as it can be seen in Figure 2 , $\mathrm{K}_{\mathrm{c}}$ has the opposite sign of $\nu$ and hence is negative in the usual situations.
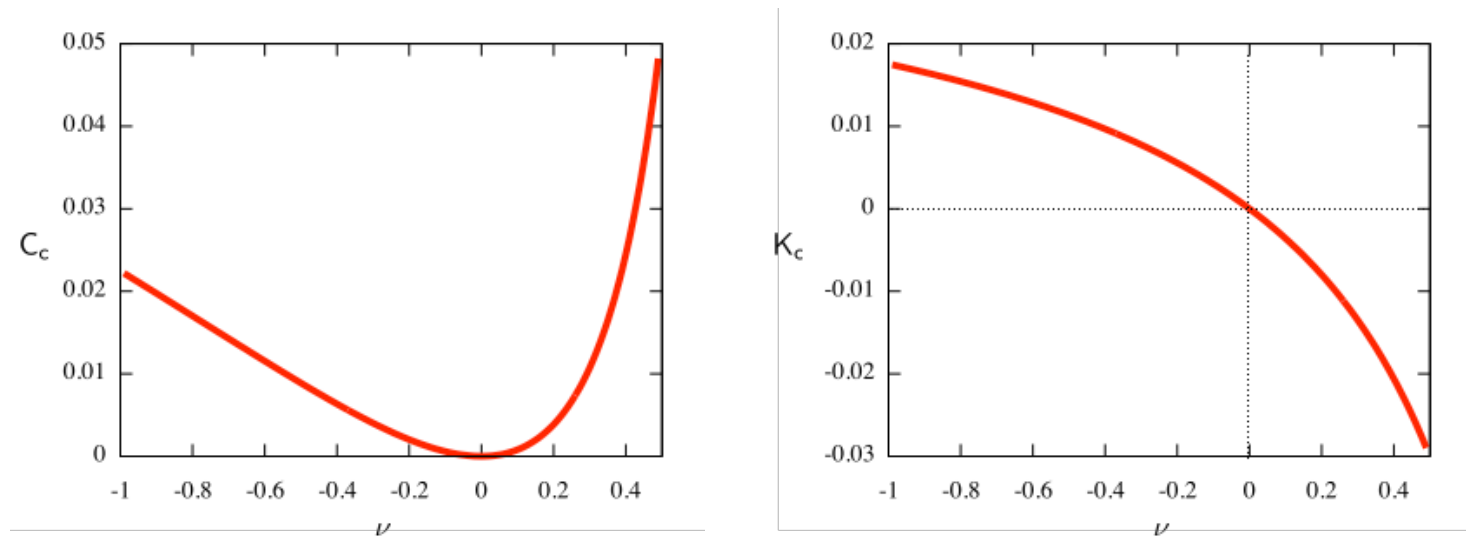

Fig. 2 Graphs of the two coefficients $\mathrm{C}_{\mathrm{c}}$ and $\mathrm{K}_{\mathrm{c}}$ in function of the Poisson ratio $\nu$, they represent the trace of the limit rotation and the (trace of the) limit displacement which correspond to the outer, macroscopic, bulk solution. Note that $\mathrm{C}_{\mathrm{c}}$ is always non-negative whereas $\mathrm{K}_{\mathrm{c}}$ changes sign with $\nu$.

At the opposite side $\bar{x}_{1}=+1 / 2$, one can proceed in the same manner and the boundary conditions read as

$$
U^{0}=U^{1}=U_{, 1}^{0}=0, \quad \mathrm{D} U_{, 1}^{1}=\mathrm{C}_{\mathrm{c}} \mathcal{M}_{11}^{0} \quad \text { at } \quad \bar{x}_{1}=+1 / 2 .
$$

One can summarise the results obtained in the present section by the following proposition:

Proposition 1 On the clamped sides $\bar{x}_{1}= \pm 1 / 2$ the displacement $U^{0}$ must satisfy the usual conditions

$$
U^{0}=\frac{\partial U^{0}}{\partial n}=0
$$

where the index $n$ refers to the outer normal $\mathbf{n}= \pm \mathbf{e}_{1}$.

The displacement $U^{1}$ must satisfy the following boundary conditions :

$$
U^{1}=0, \quad \mathrm{D} \frac{\partial U^{1}}{\partial n}=\mathrm{C}_{\mathrm{c}} \mathcal{M}_{n n}^{0}
$$

which differ from the usual ones because of the presence of a boundary layer. That requires to solve the bending boundary layer problem (35) to obtain the positive coefficient $\mathrm{C}_{\mathrm{c}}$. 


\section{Study of the boundary layer near the free sides of the plate}

\subsection{Notations}

Here again, it is sufficient to study the boundary layer near one of the two free sides. So, we consider the side $\bar{x}_{2}=-\bar{\ell} / 2$ and set

$$
y_{2}=\frac{2 \bar{x}_{2}+\bar{\ell}}{2 \eta} .
$$

We use notations similar to those of the previous section except that the directions 1 and 2 are inverted. Specifically, $\mathbf{y}=\left(y_{2}, y_{3}\right)$ represents now the small scale coordinates whereas $\bar{x}_{1}$ is the large scale coordinate. The boundary layer problems will be posed on the semi-infinite strip $\mathrm{Y}=(0,+\infty) \times(0,+1 / 2)$ of the $\left(y_{2}, y_{3}\right)$ plane and $\bar{x}_{1}$ will only play the role of a parameter varying in $I_{1}=(-1 / 2,1 / 2)$. When $\mathbf{v}=\mathbf{v}\left(\bar{x}_{1}, \mathbf{y}\right)$ is a vector field defined in $I_{1} \times \mathrm{Y}$, we now denote by $\varepsilon_{\mathbf{y}}(\mathbf{v})$ and $\varepsilon_{\bar{x}}(\mathbf{v})$ the tensor fields whose matrix representations in the basis $\left(\mathbf{e}_{1}, \mathbf{e}_{2}, \mathbf{e}_{3}\right)$ are

$$
\boldsymbol{\varepsilon}_{\mathbf{y}}(\mathbf{v})=\left(\begin{array}{ccc}
0 & \frac{1}{2} \frac{\partial v_{1}}{\partial y_{2}} & \frac{1}{2} \frac{\partial v_{1}}{\partial y_{3}} \\
\frac{1}{2} \frac{\partial v_{1}}{\partial y_{2}} & \frac{\partial v_{2}}{\partial y_{2}} & \frac{1}{2}\left(\frac{\partial v_{3}}{\partial y_{2}}+\frac{\partial v_{2}}{\partial y_{3}}\right) \\
\frac{1}{2} \frac{\partial v_{1}}{\partial y_{3}} \frac{1}{2}\left(\frac{\partial v_{3}}{\partial y_{2}}+\frac{\partial v_{2}}{\partial y_{3}}\right) & \frac{\partial v_{3}}{\partial y_{3}}
\end{array}\right) \text { and } \varepsilon_{\bar{x}}(\mathbf{v})=\left(\begin{array}{ccc}
\frac{\partial v_{1}}{\partial \bar{x}_{1}} & \frac{1}{2} \frac{\partial v_{2}}{\partial \bar{x}_{1}} & \frac{1}{2} \frac{\partial v_{3}}{\partial \bar{x}_{1}} \\
\frac{1}{2} \frac{\partial v_{2}}{\partial \bar{x}_{1}} & 0 & 0 \\
\frac{1}{2} \frac{\partial v_{3}}{\partial \bar{x}_{1}} & 0 & 0
\end{array}\right)
$$

When $\boldsymbol{\tau}=\boldsymbol{\tau}\left(\bar{x}_{1}, \mathbf{y}\right)$ is a symmetric two tensor field defined in $I_{1} \times \mathrm{Y}$, we denote by $\boldsymbol{d i v}_{\mathbf{y}} \boldsymbol{\tau}$ and $\boldsymbol{d i v}_{\bar{x}} \boldsymbol{\tau}$ the vectors fields whose representations in the basis $\left(\mathbf{e}_{1}, \mathbf{e}_{2}, \mathbf{e}_{3}\right)$ are

$$
\operatorname{div}_{\mathbf{y}} \boldsymbol{\tau}=\left(\frac{\partial \tau_{i 2}}{\partial y_{2}}+\frac{\partial \tau_{i 3}}{\partial y_{3}}\right) \mathbf{e}_{i}, \quad \operatorname{div}_{\bar{x}} \boldsymbol{\tau}=\frac{\partial \tau_{i 1}}{\partial \bar{x}_{1}} \mathbf{e}_{i}
$$

The lemma that characterises a displacement field $\mathbf{v}$ whose associated strain field $\varepsilon_{\mathbf{y}}(\mathbf{v})$ vanishes, reads now

Lemma 2 If a vector field $\mathbf{v}$ is such that $\varepsilon_{\mathbf{y}}(\mathbf{v})=0$ in $I_{1} \times \mathrm{Y}$ (in the sense of (40)), then $\mathbf{v}$ is necessarily of the form

$$
\mathbf{v}\left(\bar{x}_{1}, \mathbf{y}\right)=\mathbf{V}\left(\bar{x}_{1}\right)+\omega\left(\bar{x}_{1}\right) \mathbf{e}_{1} \wedge \mathbf{y}
$$

Furthermore, if $v_{1}=v_{2}=0$ at $y_{3}=0$, then $\mathbf{V}\left(\bar{x}_{1}\right)=V\left(\bar{x}_{1}\right) \mathbf{e}_{3}$.

4.2 Hypothesis on the form of the expansions and matching conditions

We suppose that, near the side $\bar{x}_{2}=-\bar{\ell} / 2$ of the plate, the displacement $\mathbf{u}^{\eta}$ admits the following asymptotic expansion with respect to the small parameter $\eta$ :

$$
\mathbf{u}^{\eta}\left(\overline{\mathbf{x}}, y_{3}\right)=\mathbf{v}^{0}\left(\bar{x}_{1}, \mathbf{y}\right)+\eta \mathbf{v}^{1}\left(\bar{x}_{1}, \mathbf{y}\right)+\eta^{2} \mathbf{v}^{2}\left(\bar{x}_{1}, \mathbf{y}\right)+\eta^{3} \mathbf{v}^{3}\left(\bar{x}_{1}, \mathbf{y}\right)+\cdots,
$$

with $\bar{x}_{1} \in I_{1}, \mathbf{y}=\left(y_{2}, y_{3}\right) \in \mathrm{Y}$ and $y_{2}$ related to $\bar{x}_{2}$ by (39). Consequently, stresses admit the same type of asymptotic expansion which a priori starts at order -2 ,

$$
\boldsymbol{\sigma}^{\eta}\left(\overline{\mathbf{x}}, y_{3}\right)=\eta^{-2} \boldsymbol{\tau}^{-2}\left(\bar{x}_{1}, \mathbf{y}\right)+\eta^{-1} \boldsymbol{\tau}^{-1}\left(\bar{x}_{1}, \mathbf{y}\right)+\boldsymbol{\tau}^{0}\left(\bar{x}_{1}, \mathbf{y}\right)+\eta \boldsymbol{\tau}^{1}\left(\bar{x}_{1}, \mathbf{y}\right)+\eta^{2} \boldsymbol{\tau}^{2}\left(\bar{x}_{1}, \mathbf{y}\right)+\cdots
$$

These expansions must match those introduced in Section 2.3. The first matching conditions read now as

$$
\left\{\begin{array}{l}
\mathbf{u}^{0}\left(\bar{x}_{1},-\bar{\ell} / 2, y_{3}\right)=\lim _{y_{2} \rightarrow \infty} \mathbf{v}^{0}\left(\bar{x}_{1}, \mathbf{y}\right) \\
\mathbf{u}^{1}\left(\bar{x}_{1},-\bar{\ell} / 2, y_{3}\right)=\lim _{y_{2} \rightarrow \infty}\left(\mathbf{v}^{1}\left(\bar{x}_{1}, \mathbf{y}\right)-y_{2} \mathbf{u}_{, 2}^{0}\left(\bar{x}_{1},-\bar{\ell} / 2, y_{3}\right)\right) \\
\mathbf{u}^{2}\left(\bar{x}_{1},-\bar{\ell} / 2, y_{3}\right)=\lim _{y_{2} \rightarrow \infty}\left(\mathbf{v}^{2}\left(\bar{x}_{1}, \mathbf{y}\right)-y_{2} \mathbf{u}_{, 2}^{1}\left(\bar{x}_{1},-\bar{\ell} / 2, y_{3}\right)-\frac{1}{2} y_{2}^{2} \mathbf{u}_{, 22}^{0}\left(\bar{x}_{1},-\bar{\ell} / 2, y_{3}\right)\right)
\end{array}\right.
$$

The same type of matching conditions hold for stresses, except that they start at order -2 . 
4.3 Determination of $\mathbf{v}^{0}$ and $\mathbf{v}^{1}$

Inserting (41)-(42) into (6)-(9) gives for the leading terms the following set of equations

$$
\left\{\begin{array}{lr}
\operatorname{div}_{\mathbf{y}} \boldsymbol{\tau}^{-2}=\mathbf{0} & \text { in } I_{1} \times \mathrm{Y}, \\
\varepsilon_{\mathbf{y}}\left(\mathbf{v}^{0}\right)=-\nu \operatorname{Tr} \boldsymbol{\tau}^{-2} \mathbf{I}+(1+\nu) \boldsymbol{\tau}^{-2} & \text { in } I_{1} \times \mathrm{Y}, \\
\boldsymbol{\tau}^{-2} \mathbf{e}_{2}=\mathbf{0} \text { at } y_{2}=0, \quad v_{1}^{0}=v_{2}^{0}=\tau_{33}^{-2}=0 \text { at } y_{3}=0, \quad \boldsymbol{\tau}^{-2} \mathbf{e}_{3}=\mathbf{0} & \text { at } y_{3}=1 / 2 .
\end{array}\right.
$$

Moreover, since $\boldsymbol{\sigma}^{-2}=\mathbf{0}$, the first matching condition for stresses gives $\boldsymbol{\tau}^{-2}\left(\bar{x}_{1},+\infty, y_{3}\right)=0$. Multiplying the equilibrium equation by $\mathbf{v}^{0}$ and integrating over $I_{1} \times \mathrm{Y}$ leads to $\int_{I_{1} \times \mathrm{Y}} \operatorname{div}_{\mathbf{y}} \boldsymbol{\tau}^{-2} \cdot \mathbf{v}^{0} d \bar{x}_{1} d \mathbf{y}=0$. Integrating by parts the integral, using boundary conditions, and matching conditions for $\boldsymbol{\tau}^{-2}$ gives $\int_{I_{1} \times \mathrm{Y}} \boldsymbol{\tau}^{-2} \cdot \boldsymbol{\varepsilon}_{\mathbf{y}}\left(\mathbf{v}^{0}\right) d \bar{x}_{1} d \mathbf{y}=0$. Then, by the constitutive relations between $\tau^{-2}$ and $\varepsilon_{\mathbf{y}}\left(\mathbf{v}^{0}\right)$, one can conclude that

$$
\boldsymbol{\tau}^{-2}=\mathbf{0}, \quad \varepsilon_{\mathbf{y}}\left(\mathbf{v}^{0}\right)=\mathbf{0} \quad \text { in } I_{1} \times \mathrm{Y} .
$$

Therefore, Lemma 2 gives $\mathbf{v}^{0}=V^{0}\left(\bar{x}_{1}\right) \mathbf{e}_{3}+\omega^{0}\left(\bar{x}_{1}\right) \mathbf{e}_{1} \wedge \mathbf{y}$. Then, using the first matching condition (43) with (15) gives $V^{0}\left(\bar{x}_{1}\right)=U^{0}\left(\bar{x}_{1},-\bar{\ell} / 2\right), \omega^{0}\left(\bar{x}_{1}\right)=0$ and finally

$$
\mathbf{v}^{0}\left(\bar{x}_{1}, \mathbf{y}\right)=U^{0}\left(\bar{x}_{1},-\bar{\ell} / 2\right) \mathbf{e}_{3} \quad \text { in } \quad I_{1} \times \mathrm{Y} .
$$

Let us set $\mathbf{v}^{*}\left(\bar{x}_{1}, \mathbf{y}\right)=U_{, 1}^{0}\left(\bar{x}_{1},-\bar{\ell} / 2\right) y_{3} \mathbf{e}_{1}$ and remark that $\boldsymbol{\varepsilon}_{\bar{x}}\left(\mathbf{v}^{0}\right)=\boldsymbol{\varepsilon}_{\mathbf{y}}\left(\mathbf{v}^{*}\right)$. Considering now the next terms of the expansion, one deduces from (6)-(9) and (44) that $\boldsymbol{\tau}^{-1}$ and $\mathbf{v}^{1}$ must satisfy

$$
\left\{\begin{array}{lr}
\operatorname{div}_{\mathbf{y}} \boldsymbol{\tau}^{-1}=\mathbf{0} & \text { in } I_{1} \times \mathrm{Y}, \\
\boldsymbol{\varepsilon}_{\mathbf{y}}\left(\mathbf{v}^{1}+\mathbf{v}^{*}\right)=-\nu \operatorname{Tr} \boldsymbol{\tau}^{-1} \mathbf{I}+(1+\nu) \boldsymbol{\tau}^{-1} & \text { in } I_{1} \times \mathrm{Y}, \\
\boldsymbol{\tau}^{-1} \mathbf{e}_{2}=\mathbf{0} \text { at } y_{2}=0, \quad v_{1}^{1}=v_{2}^{1}=\tau_{33}^{-1}=0 \text { at } y_{3}=0, \quad \boldsymbol{\tau}^{-1} \mathbf{e}_{3}=\mathbf{0} & \text { at } y_{3}=1 / 2 .
\end{array}\right.
$$

Since $\boldsymbol{\sigma}^{-1}=\mathbf{0}$ and since $\mathbf{v}^{*}$ satisfies the boundary condition at $y_{3}=0$, one can follow the same procedure as for $\mathbf{v}^{0}$ and $\boldsymbol{\tau}^{-2}$ to obtain $\int_{I_{1} \times \mathrm{Y}} \boldsymbol{\tau}^{-1} \cdot \varepsilon_{\mathbf{y}}\left(\mathbf{v}^{1}+\mathbf{v}^{*}\right) d \bar{x}_{1} d \mathbf{y}=0$ and hence

$$
\varepsilon_{\mathbf{y}}\left(\mathbf{v}^{1}+\mathbf{v}^{*}\right)=\mathbf{0}, \quad \boldsymbol{\tau}^{-1}=\mathbf{0} \quad \text { in } \quad I_{1} \times \mathrm{Y} .
$$

Therefore, by Lemma $2, \mathbf{v}^{1}+\mathbf{v}^{*}=V^{1}\left(\bar{x}_{1}\right) \mathbf{e}_{3}+\omega^{1}\left(\bar{x}_{1}\right) \mathbf{e}_{1} \wedge \mathbf{y}$. Then, using (15), (16) and (45), the second matching condition (43) reads

$$
-y_{3} U_{, 2}^{0}\left(\bar{x}_{1},-\bar{\ell} / 2\right) \mathbf{e}_{2}+U^{1}\left(\bar{x}_{1},-\bar{\ell} / 2\right) \mathbf{e}_{3}=\lim _{y_{2} \rightarrow+\infty}\left(V^{1}\left(\bar{x}_{1}\right) \mathbf{e}_{3}+\omega^{1}\left(\bar{x}_{1}\right) \mathbf{e}_{1} \wedge \mathbf{y}-y_{2} U_{, 2}^{0}\left(\bar{x}_{1},-\bar{\ell} / 2\right) \mathbf{e}_{3}\right),
$$

and hence is satisfied if and only if $V^{1}\left(\bar{x}_{1}\right)=U^{1}\left(\bar{x}_{1},-\bar{\ell} / 2\right)$ and $\omega^{1}\left(\bar{x}_{1}\right)=U_{, 2}^{0}\left(\bar{x}_{1},-\bar{\ell} / 2\right)$. Therefore $\mathbf{v}^{1}$ has the following form

$$
\mathbf{v}^{1}\left(\bar{x}_{1}, \mathbf{y}\right)=-U_{, \alpha}^{0}\left(\bar{x}_{1},-\bar{\ell} / 2\right) y_{3} \mathbf{e}_{\alpha}+\left(U^{1}\left(\bar{x}_{1},-\bar{\ell} / 2\right)+y_{2} U_{, 2}^{0}\left(\bar{x}_{1},-\bar{\ell} / 2\right)\right) \mathbf{e}_{3} .
$$

At this stage, we have not obtained boundary conditions for $U^{0}$ and $U^{1}$. The first ones are given by the problem governing $\mathbf{v}^{2}$ and $\tau^{0}$.

4.4 First boundary condition for $U^{0}$

Using the previous results, one deduces from (6)-(9) that $\boldsymbol{\tau}^{0}$ and $\mathbf{v}^{2}$ must satisfy

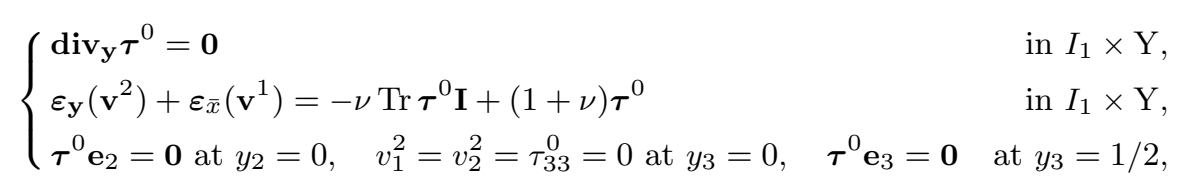

and the condition at infinity for $\tau^{0}$ which is given by the matching condition between $\sigma^{0}$ and $\tau^{0}$, i.e. ,

$$
\lim _{y_{2} \rightarrow+\infty} \boldsymbol{\tau}^{0}\left(\bar{x}_{1}, \mathbf{y}\right)=\boldsymbol{\sigma}^{0}\left(\bar{x}_{1},-\bar{\ell} / 2, y_{3}\right) .
$$

Since rigid motions of the form $\mathbf{v}\left(\bar{x}_{1}, \mathbf{y}\right)=V\left(\bar{x}_{1}\right) \mathbf{e}_{3}+\omega\left(\bar{x}_{1}\right) \mathbf{e}_{1} \wedge \mathbf{y}$ are still allowed by virtue of Lemma 2 , the loading at infinity given by $\boldsymbol{\sigma}^{0}$ must satisfy conditions in order that this problem for $\mathbf{v}^{2}$ and $\boldsymbol{\tau}^{0}$ admits at least 
one solution. Specifically, the $\mathbf{e}_{3}$ component of the resultant force and the $\mathbf{e}_{1}$ component of the resultant moment of the external forces must vanish. This leads to the following two conditions

$$
\int_{\partial \mathrm{Y}} \boldsymbol{\tau}^{0} \mathbf{n} \cdot \mathbf{e}_{3} d s=0, \quad \int_{\partial \mathrm{Y}}\left(\mathbf{y} \wedge \boldsymbol{\tau}^{0} \mathbf{n}\right) \cdot \mathbf{e}_{1} d s=0,
$$

where $\mathbf{n}$ denotes the outer normal to $\mathrm{Y}$ and $s$ is the arclength along $\partial \mathrm{Y}$. By virtue of the boundary conditions satisfied by $\tau^{0}$, the first equality is automatically satisfied whereas the second one is reduced to

$$
\int_{0}^{1 / 2} y_{3} \sigma_{22}^{0}\left(\bar{x}_{1},-\bar{\ell} / 2, y_{3}\right) d y_{3}=0 .
$$

To obtain the above equality one assumes that the decay of $\tau_{23}^{0}$ to 0 is exponential. Comparing with (10), this condition requires that the normal bending moment $\mathcal{M}_{22}^{0}$ vanishes

$$
\mathcal{M}_{22}^{0}=0 \quad \text { at } \quad \bar{x}_{2}=-\bar{\ell} / 2
$$

a condition that can be also expressed in terms of $U^{0}$ using (20). Specifically, $U_{, 22}^{0}+\nu U_{, 11}^{0}=0$ at $\bar{x}_{2}=-\bar{\ell} / 2$ which implies that $\sigma_{22}^{0}=0$ at $\bar{x}_{2}=-\bar{\ell} / 2$.

4.5 Determination of $\mathbf{v}^{2}$ and $\tau^{0}$

Assuming that the condition (49) is satisfied, the problem (47)-(48) admits a solution which is defined up to an admissible rigid displacement. This problem can be decomposed into an in-plane problem and an out-of-plane problem (the plane being the plane $\left(y_{2}, y_{3}\right)$ of Y). For the in-plane problem, since all the plane components of $\varepsilon_{\bar{x}}\left(\mathbf{v}^{1}\right)$ and $\boldsymbol{\sigma}^{0}$ vanish, it is easy to check that the planar components of $\boldsymbol{\tau}^{0}$ vanish. Hence,

$$
\tau_{22}^{0}=\tau_{23}^{0}=\tau_{33}^{0}=0, \quad \tau_{11}^{0}\left(\bar{x}_{1}, \mathbf{y}\right)=-y_{3} U_{, 11}^{0}\left(\bar{x}_{1},-\bar{\ell} / 2\right)
$$

The compatibility conditions with the help of the boundary condition at $y_{3}=0$ give the plane components of $\mathbf{v}^{2}$ up to a rigid motion which is fixed by the third matching condition (43). Finally, one gets

$$
\left\{\begin{array}{l}
v_{2}^{2}\left(\bar{x}_{1}, \mathbf{y}\right)=-y_{3} U_{, 2}^{1}\left(\bar{x}_{1},-\bar{\ell} / 2\right)+\nu y_{2} y_{3} U_{, 11}^{0}\left(\bar{x}_{1},-\bar{\ell} / 2\right), \\
v_{3}^{2}\left(\bar{x}_{1}, \mathbf{y}\right)=U^{2}\left(\bar{x}_{1},-\bar{\ell} / 2\right)+y_{2} U_{, 2}^{1}\left(\bar{x}_{1},-\bar{\ell} / 2\right)+\frac{\nu}{2}\left(y_{3}^{2}-y_{2}^{2}\right) U_{, 11}^{0}\left(\bar{x}_{1},-\bar{\ell} / 2\right) .
\end{array}\right.
$$

The out-of-plane problem reads

$$
\left\{\begin{array}{l}
\frac{\partial \tau_{12}^{0}}{\partial y_{2}}+\frac{\partial \tau_{13}^{0}}{\partial y_{3}}=0, \\
2(1+\nu) \tau_{12}^{0}=\frac{\partial v_{1}^{2}}{\partial y_{2}}-y_{3} U_{, 12}^{0}, \\
2(1+\nu) \tau_{13}^{0}=\frac{\partial v_{1}^{2}}{\partial y_{3}}+y_{2} U_{, 12}^{0}+U_{, 1}^{1},
\end{array} \quad \text { in } I_{1} \times \mathrm{Y}, \quad\left\{\begin{array}{l}
\tau_{12}^{0}=0 \text { at } y_{2}=0 \\
v_{1}^{2}=0 \text { at } y_{3}=0 \\
\tau_{13}^{0}=0 \text { at } y_{3}=1 / 2, \\
\tau_{12}^{0}=-\frac{y_{3}}{1+\nu} U_{, 12}^{0} \text { at } y_{2}=+\infty \\
\tau_{13}^{0}=0 \text { at } y_{2}=+\infty
\end{array}\right.\right.
$$

where the values of $U^{0}, U^{1}$ and their derivatives are taken at $\left(\bar{x}_{1},-\bar{\ell} / 2\right)$. It admits a unique solution which by linearity can be written as follows

$$
\left\{\begin{array}{l}
v_{1}^{2}\left(\bar{x}_{1}, \mathbf{y}\right)=-y_{3} U_{, 1}^{1}\left(\bar{x}_{1},-\bar{\ell} / 2\right)+\left(\mathbf{W}^{\mathrm{f}}(\mathbf{y})-y_{2} y_{3}\right) U_{, 12}^{0}\left(\bar{x}_{1},-\bar{\ell} / 2\right), \\
\tau_{12}^{0}\left(\bar{x}_{1}, \mathbf{y}\right)=\frac{U_{, 12}^{0}\left(\bar{x}_{1},-\bar{\ell} / 2\right)}{2(1+\nu)}\left(\frac{\partial \mathbf{W}^{\mathrm{f}}}{\partial y_{2}}(\mathbf{y})-2 y_{3}\right) \\
\tau_{13}^{0}\left(\bar{x}_{1}, \mathbf{y}\right)=\frac{U_{, 12}^{0}\left(\bar{x}_{1},-\bar{\ell} / 2\right)}{2(1+\nu)} \frac{\partial \mathbf{W}^{\mathrm{f}}}{\partial y_{3}}(\mathbf{y})
\end{array}\right.
$$

where $W^{f}$ is the scalar field solution of the Laplacian problem posed in $Y$, called the twist boundary layer problem at a free side,

$$
\left\{\begin{array}{l}
\Delta \mathbf{W}^{\mathrm{f}}=0 \quad \text { in } \quad \mathrm{Y} \\
\frac{\partial \mathbf{W}^{\mathrm{f}}}{\partial y_{2}}\left(0, y_{3}\right)=2 y_{3}, \quad \mathbf{W}^{\mathrm{f}}\left(y_{2}, 0\right)=0, \quad \frac{\partial \mathbf{W}^{\mathrm{f}}}{\partial y_{3}}\left(y_{2}, 1 / 2\right)=0 \\
\lim _{y_{2} \rightarrow+\infty} \mathbf{W}^{\mathrm{f}}(\mathbf{y})=0
\end{array}\right.
$$


Note that this problem is universal in the sense that it is free from any parameter. A boundary layer appears at the free sides $\bar{x}_{2}= \pm \bar{\ell} / 2$ if $U_{, 12}^{0}$ is non-zero at those sides because $\sigma_{12}^{0}$ does not satisfy the boundary condition of vanishing shear. Accordingly, the shear decreases exponentially to zero in the boundary layer. The solution $\mathrm{W}^{\mathrm{f}}$ can be expressed under the form of the following series

$$
\mathbf{W}^{\mathrm{f}}\left(y_{2}, y_{3}\right)=\sum_{n \in \mathbb{N}} \frac{8(-1)^{n+1}}{(2 n+1)^{3} \pi^{3}} \exp \left(-(2 n+1) \pi y_{2}\right) \sin (2 n+1) \pi y_{3} .
$$

We can see in Figure 3 that the thickness of the boundary layer is about 1-1.5 and hence, by comparison with Figure 1, larger than that of the bending boundary layer problem (35). Let us note the following property for $\mathrm{W}^{\mathrm{f}}$ which will be useful:

$$
\int_{0}^{+\infty} \mathbf{W}^{\mathrm{f}}\left(y_{2}, 1 / 2\right) d y_{2}=\int_{\mathrm{Y}} \frac{\partial \mathbf{W}^{\mathrm{f}}}{\partial y_{3}}(\mathbf{y}) d \mathbf{y}=-\frac{1}{12}
$$

Indeed, from $\Delta \mathrm{W}^{\mathrm{f}}=0$ and using the boundary conditions satisfied by $\mathrm{W}^{\mathrm{f}}$, one gets

$$
0=-\int_{\mathrm{Y}} y_{3} \Delta \mathrm{W}^{\mathrm{f}} d \mathbf{y}=\left.\int_{0}^{1 / 2} y_{3} \frac{\partial \mathrm{W}^{\mathrm{f}}}{\partial y_{2}}\right|_{y_{2}=0} d y_{3}+\int_{\mathrm{Y}} \frac{\partial \mathrm{W}^{\mathrm{f}}}{\partial y_{3}} d \mathbf{y}=\frac{1}{12}+\int_{\mathrm{Y}} \frac{\partial \mathrm{W}^{\mathrm{f}}}{\partial y_{3}} d \mathbf{y}
$$

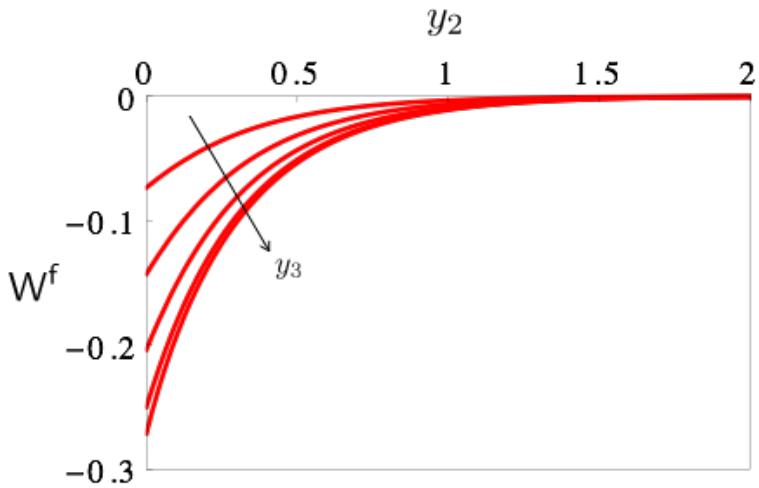

Fig. $3 \mathrm{~W}^{\mathrm{f}}$ is a universal twist boundary layer inducing an out-of-plane displacement near the free side which allows to satisfy zero transverse shear at the boundary. In the graph above, we plot $\mathrm{W}^{\mathrm{f}}$ as a function of $y_{2}$ for five values of $y_{3}, y_{3}=0.1,0.2,0.3,0.4,0.5$.

4.6 Second boundary condition for $U^{0}$ and first boundary condition for $U^{1}$

Using the previous results, one deduces from (6)-(9) that $\boldsymbol{\tau}^{1}$ and $\mathbf{v}^{3}$ must satisfy

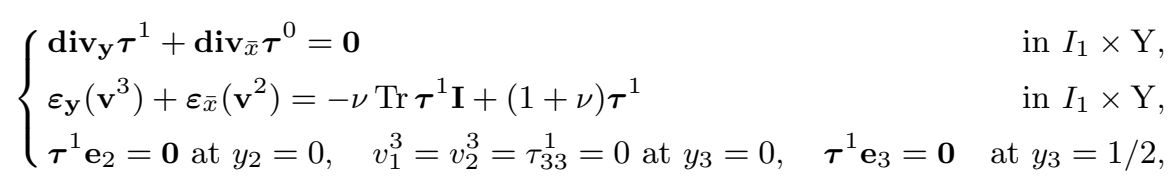

and the condition at infinity for $\tau^{1}$ which is given by the matching condition, i.e. ,

$$
\lim _{y_{2} \rightarrow+\infty}\left(\boldsymbol{\tau}^{1}\left(\bar{x}_{1}, \mathbf{y}\right)-y_{2} \boldsymbol{\sigma}_{, 2}^{0}\left(\bar{x}_{1},-\bar{\ell} / 2, y_{3}\right)\right)=\boldsymbol{\sigma}^{1}\left(\bar{x}_{1},-\bar{\ell} / 2, y_{3}\right)
$$

This problem admits a solution only if the resultant of the external forces in the direction $\mathbf{e}_{3}$ vanishes and if the resultant torque of the external forces in the direction $\mathbf{e}_{1}$ vanishes. The former condition gives the second boundary condition for $U^{0}$ whereas the latter gives the first boundary condition for $U^{1}$. To simplify the notation, we omit up to the end of this section the arguments of the functions when there is no risk of confusion. In particular, all global quantities are implicitly evaluated at $\left(\bar{x}_{1},-\bar{\ell} / 2\right)$.

First, multiplying the equilibrium equation by $\mathbf{e}_{3}$ and integrating over $\mathrm{Y}$ at a given $\bar{x}_{1}$ leads to

$$
0=\int_{\left\{\bar{x}_{1}\right\} \times \mathrm{Y}} 2\left(\frac{\partial \tau_{32}^{1}}{\partial y_{2}}+\frac{\partial \tau_{33}^{1}}{\partial y_{3}}+\frac{\partial \tau_{31}^{0}}{\partial \bar{x}_{1}}\right) d \mathbf{y}=\int_{0}^{1 / 2} 2 \tau_{32}^{1}\left(\bar{x}_{1},+\infty, y_{3}\right) d y_{3}+\frac{U_{, 112}^{0}}{1+\nu} \int_{\mathrm{Y}} \frac{\partial \mathbf{W}^{\mathrm{f}}}{\partial y_{3}} d \mathbf{y}
$$


where have been used the boundary conditions for $\tau_{33}^{1}$ at $y_{3}=0$ and $y_{3}=1 / 2$, the boundary condition for $\tau_{32}^{1}$ at $y_{2}=0$, and the expression (50) for $\tau_{31}^{0}$. Then, since $\tau_{32}^{1}$ at $y_{2}=+\infty$ is equal to $\sigma_{32}^{1}$ at $\bar{x}_{2}=-\bar{\ell} / 2$, one gets the desired condition

$$
\int_{0}^{1 / 2} 2 \sigma_{32}^{1}\left(\bar{x}_{1},-\bar{\ell} / 2, y_{3}\right) d y_{3}+\frac{U_{, 112}^{0}}{1+\nu}\left(\int_{\mathrm{Y}} \frac{\partial \mathrm{W}^{\mathrm{f}}}{\partial y_{3}} d \mathbf{y}\right)=0 .
$$

Using (10), (20) and (53), this condition reads also as

$$
\mathcal{T}_{2}^{0}-\mathcal{M}_{21,1}^{0}=0 \quad \text { at } \quad \bar{x}_{2}=-\bar{\ell} / 2
$$

which, expressed in terms of $U^{0}$, becomes $U_{, 222}^{0}+(2-\nu) U_{, 112}^{0}=0$.

Now, multiplying the equilibrium equation by $\mathbf{e}_{1} \wedge \mathbf{y}$ and integrating, at a given $\bar{x}_{1}$, with respect to $\mathbf{y}$ over $\mathrm{Y}_{\zeta}=(0, \zeta) \times(0,1 / 2)($ where $\zeta>0$ is destined to tend to infinity) leads to

$$
0=\int_{\left\{\bar{x}_{1}\right\} \times \mathrm{Y}_{\zeta}} 2 \operatorname{div}_{\mathbf{y}} \boldsymbol{\tau}^{1} \cdot\left(\mathbf{e}_{1} \wedge \mathbf{y}\right) d \mathbf{y}+\frac{U_{, 112}^{0}}{1+\nu} \int_{\mathrm{Y}_{\zeta}}\left(y_{2} \frac{\partial \mathrm{W}^{\mathrm{f}}}{\partial y_{3}}-y_{3} \frac{\partial \mathrm{W}^{\mathrm{f}}}{\partial y_{2}}+2 y_{3}^{2}\right) d \mathbf{y},
$$

where we have used the expression (50) for $\tau^{0}$. Integrating by parts the first integral, using the expression (20) of $\mathcal{M}_{21}^{0}$ and the boundary conditions get

$$
2 \int_{0}^{1 / 2} y_{3} \tau_{22}^{1}\left(\bar{x}_{1}, \zeta, y_{3}\right) d y_{3}=2 \zeta \int_{0}^{1 / 2} \tau_{32}^{1}\left(\bar{x}_{1}, \zeta, y_{3}\right) d y_{3}+\zeta \mathcal{M}_{21,1}^{0}+12 \mathcal{M}_{21,1}^{0} \int_{\mathrm{Y}_{\zeta}}\left(y_{2} \frac{\partial \mathbf{W}^{\mathrm{f}}}{\partial y_{3}}-y_{3} \frac{\partial \mathbf{W}^{\mathrm{f}}}{\partial y_{2}}\right) d \mathbf{y} .
$$

The matching condition (54) gives

$$
\lim _{\zeta \rightarrow+\infty}\left(\int_{0}^{1 / 2} 2 y_{3} \tau_{22}^{1}\left(\bar{x}_{1}, \zeta, y_{3}\right) d y_{3}+\zeta \mathcal{M}_{22,2}^{0}\right)=-\mathcal{M}_{22}^{1}, \quad \lim _{\zeta \rightarrow+\infty} \int_{0}^{1 / 2} 2 \tau_{32}^{1}\left(\bar{x}_{1}, \zeta, y_{3}\right) d y_{3}=\mathcal{T}_{2}^{0} .
$$

whereas the equilibrium equation (11) at order 0 gives $\mathcal{T}_{2}^{0}+\mathcal{M}_{21,1}^{0}+\mathcal{M}_{22,2}^{0}=0$. Because convergence is exponential, after inserting these relations into (55) one finally obtains

$$
\mathcal{M}_{22}^{1}=6 \mathrm{~J}_{\mathrm{f}} \mathcal{M}_{21,1}^{0} \quad \text { at } \quad \bar{x}_{2}=-\bar{\ell} / 2
$$

where $J_{f}$ is the coefficient defined by

$$
\mathrm{J}_{\mathrm{f}}=2 \int_{\mathrm{Y}}\left(y_{3} \frac{\partial \mathrm{W}^{\mathrm{f}}}{\partial y_{2}}-y_{2} \frac{\partial \mathrm{W}^{\mathrm{f}}}{\partial y_{3}}\right) d \mathbf{y}
$$

Therefore, the normal bending moment does not satisfy in general the free boundary condition because of the boundary layer associated with the shear stress $\sigma_{12}$. The coefficient $\mathrm{J}_{\mathrm{f}}$ is obtained by solving the twist boundary layer problem (51). Since this problem is universal, so is the coefficient $J_{f}$. Let us prove that $J_{f}$ reads also as $\mathrm{J}_{\mathrm{f}}=2 \int_{\mathrm{Y}} \nabla \mathrm{W}^{\mathrm{f}} \cdot \nabla \mathrm{W}^{\mathrm{f}} d \mathbf{y}$ and hence is positive. Indeed, from (51) one gets

$$
0=-\int_{\mathrm{Y}}\left(\mathbf{W}^{\mathrm{f}}+y_{2} y_{3}\right) \Delta \mathbf{W}^{\mathrm{f}} d \mathbf{y}=\int_{\mathrm{Y}}\left(\nabla \mathrm{W}^{\mathrm{f}} \cdot \nabla \mathbf{W}^{\mathrm{f}}+y_{2} \frac{\partial \mathrm{W}^{\mathrm{f}}}{\partial y_{3}}+y_{3} \frac{\partial \mathrm{W}^{\mathrm{f}}}{\partial y_{2}}\right) d \mathbf{y}+\int_{0}^{1 / 2} 2 y_{3} \mathbf{W}^{\mathrm{f}}\left(0, y_{3}\right) d y_{3},
$$

and the result follows by $\int_{0}^{1 / 2} 2 y_{3} \mathrm{~W}^{\mathrm{f}}\left(0, y_{3}\right) d y_{3}=-\int_{\mathrm{Y}} 2 y_{3} \frac{\partial \mathrm{W}^{\mathrm{f}}}{\partial y_{2}} d \mathbf{y}$. Using the series (52), the computation of $\mathrm{J}_{\mathrm{f}}$ gives

$$
\mathrm{J}_{\mathrm{f}}=\sum_{n \in \mathbb{N}} \frac{32}{(2 n+1)^{5} \pi^{5}} \approx 0.105041
$$

4.7 Determination of the out-of-plane part of $\mathbf{v}^{3}$ and $\boldsymbol{\tau}^{1}$

In order to find the second boundary condition for $U^{1}$, we shall solve the out-of-plane problem for $\mathbf{v}^{3}$ and $\tau^{1}$. It reads

$$
\left\{\begin{array} { l } 
{ \frac { \partial \tau _ { 1 2 } ^ { 1 } } { \partial y _ { 2 } } + \frac { \partial \tau _ { 1 3 } ^ { 1 } } { \partial y _ { 3 } } = y _ { 3 } U _ { , 1 1 1 } ^ { 0 } , } \\
{ 2 ( 1 + \nu ) \tau _ { 1 2 } ^ { 1 } = \frac { \partial v _ { 1 } ^ { 3 } } { \partial y _ { 2 } } + \nu y _ { 2 } y _ { 3 } U _ { , 1 1 1 } ^ { 0 } - y _ { 3 } U _ { , 1 2 } ^ { 1 } , } \\
{ 2 ( 1 + \nu ) \tau _ { 1 3 } ^ { 1 } = \frac { \partial v _ { 1 } ^ { 3 } } { \partial y _ { 3 } } + \frac { \nu } { 2 } ( y _ { 3 } ^ { 2 } - y _ { 2 } ^ { 2 } ) U _ { , 1 1 1 } ^ { 0 } + y _ { 2 } U _ { , 1 2 } ^ { 1 } + U _ { , 1 } ^ { 2 } , }
\end{array} \quad \text { in } I _ { 1 } \times \mathrm { Y } , \quad \text { with } \quad \left\{\begin{array}{ll}
\tau_{12}^{1}=0 & \text { at } y_{2}=0 \\
v_{1}^{3}=0 & \text { at } y_{3}=0 \\
\tau_{13}^{1}=0 & \text { at } y_{3}=1 / 2,
\end{array}\right.\right.
$$


and matching conditions at infinity

$$
\lim _{y_{2} \rightarrow+\infty}\left(\tau_{12}^{1}-\frac{\nu y_{2} y_{3}}{1+\nu} U_{, 111}^{0}\right)=-\frac{y_{3}}{1+\nu} U_{, 12}^{1}, \quad \lim _{y_{2} \rightarrow+\infty} \tau_{13}^{1}=\frac{4 y_{3}^{2}-1}{8(1+\nu)} U_{, 111}^{0},
$$

where the values of $U^{0}, U^{1}, U^{2}$ and their derivatives are taken at $\left(\bar{x}_{1},-\bar{\ell} / 2\right)$. By linearity this problem (whose solution is unique) can be decomposed into three sub-problems: one associated with $U_{, 1}^{2}$, the second with $U_{, 12}^{1}$ and the third with $U_{, 111}^{0}$. The first two have been solved in Subsection 4.5. It turns out that the third one can be solved in closed form eventually obtaining

$$
\left\{\begin{array}{l}
v_{1}^{3}=-y_{3} U_{, 1}^{2}+\left(\mathbf{W}^{\mathrm{f}}(\mathbf{y})-y_{2} y_{3}\right) U_{, 12}^{1}+\left(\frac{\nu}{2} y_{2}^{2} y_{3}+(2-\nu) \frac{y_{3}^{3}}{6}-\frac{y_{3}}{4}\right) U_{, 111}^{0}, \\
\tau_{12}^{1}=\frac{U_{, 12}^{1}}{2(1+\nu)}\left(\frac{\partial \mathbf{W}^{\mathrm{f}}}{\partial y_{2}}(\mathbf{y})-2 y_{3}\right)+\frac{\nu y_{2} y_{3}}{1+\nu} U_{, 111}^{0}, \\
\tau_{13}^{1}=\frac{U_{, 12}^{1}}{2(1+\nu)} \frac{\partial \mathbf{W}^{\mathrm{f}}}{\partial y_{3}}(\mathbf{y})+\frac{4 y_{3}^{2}-1}{8(1+\nu)} U_{, 111}^{0} .
\end{array}\right.
$$

\subsection{Second boundary condition for $U^{1}$}

To find the second boundary condition that $U^{1}$ must satisfy at $\bar{x}_{2}=-\bar{\ell} / 2$, one follows the same procedure as that described in Section (4.6). Specifically, the problem for $\tau^{2}$ and $\mathbf{v}^{4}$ admits a solution only if the resultant of the external forces in the direction $\mathbf{e}_{3}$ vanishes and if the resultant torque of the external forces in the direction $\mathbf{e}_{1}$ vanishes. The former condition gives the second boundary condition for $U^{1}$ whereas the latter gives the first boundary condition for $U^{2}$. We only consider here the former.

Starting from the equilibrium equation in the direction 3 and integrating over $\mathrm{Y}_{\zeta}$ (at given $\bar{x}_{1}$ ) leads to

$$
0=\int_{\mathrm{Y}_{\zeta}} 2\left(\frac{\partial \tau_{32}^{2}}{\partial y_{2}}+\frac{\partial \tau_{33}^{2}}{\partial y_{3}}+\frac{\partial \tau_{31}^{1}}{\partial \bar{x}_{1}}+f\right) d \mathbf{y}
$$

where $\zeta>0$ is destined to go to infinity. Boundary conditions give $\tau_{32}^{2}=0$ at $y_{2}=0, \tau_{33}^{2}=0$ at $y_{3}=0$ and $\tau_{33}^{2}=F$ at $y_{3}=1 / 2$. Note that body forces appear here and we assume that one can define their limit at $\bar{x}_{2}=-\bar{\ell} / 2$. Accordingly, in the relations above $f$ and $F$ stand for $f\left(\bar{x}_{1},-\bar{\ell} / 2\right)$ and $F\left(\bar{x}_{1},-\bar{\ell} / 2\right)$, respectively. With the help of the expression (57) for $\tau_{13}^{1}$ one gets

$$
0=2 \int_{0}^{1 / 2} \tau_{32}^{2}\left(\zeta, y_{3}\right) d y_{3}+\frac{U_{, 112}^{1}}{1+\nu} \int_{\mathrm{Y}_{\zeta}} \frac{\partial \mathrm{W}^{\mathrm{f}}}{\partial y_{3}}(\mathbf{y}) d \mathbf{y}-\zeta \mathrm{D}(1-\nu) U_{, 1111}^{0}+\zeta \mathcal{F}^{0}
$$

Matching conditions for the stress at order 2 give

$$
\mathcal{T}_{2}^{1}=\lim _{\zeta \rightarrow \infty}\left(2 \int_{0}^{1 / 2} \tau_{32}^{2}\left(\zeta, y_{3}\right) d y_{3}-\zeta \mathcal{T}_{2,2}^{0}\right)
$$

But, by virtue of $(21)$ and $(49)$, one has $\mathrm{D}(1-\nu) U_{, 111}^{0}-\mathcal{F}^{0}=\mathcal{T}_{2,2}^{0}$. Therefore, by passing to the limit when $\zeta$ goes to infinity in (58) and using (53), one gets

$$
\mathcal{T}_{2}^{1}-\mathcal{M}_{21,1}^{1}=0 \quad \text { at } \quad \bar{x}_{2}=-\bar{\ell} / 2
$$

which is the usual boundary condition at a free side. That means that there is no boundary layer effect for this condition at this order.

At the opposite side $\bar{x}_{2}=+1 / 2$, one can proceed in the same manner and the boundary conditions read as

$$
\mathcal{M}_{22}^{0}=\mathcal{T}_{2}^{0}-\mathcal{M}_{21,1}^{0}=\mathcal{T}_{2}^{1}-\mathcal{M}_{21,1}^{1}=0, \quad \mathcal{M}_{22}^{1}=-6 \mathrm{~J}_{\mathrm{f}} \mathcal{M}_{21,1}^{0} \quad \text { at } \quad \bar{x}_{1}=+1 / 2 .
$$

One can summarise the results obtained in the present section by the following proposition:

Proposition 2 On the free sides $\bar{x}_{2}= \pm \bar{\ell} / 2$ the shear force vector $\boldsymbol{T}^{0}$ and the bending moment tensor $\mathcal{M}^{0}$ must satisfy the usual conditions

$$
\mathcal{T}_{n}^{0}-\frac{\partial \mathcal{M}_{n t}^{0}}{\partial t}=0, \quad \mathcal{M}_{n n}^{0}=0
$$

where the indices $n$ and $t$ refer to the outer normal $\mathbf{n}= \pm \mathbf{e}_{2}$ and the tangent vector $\mathbf{t}= \pm \mathbf{e}_{1}$. 
The shear force vector $\mathcal{T}^{1}$ and the bending moment tensor $\mathcal{M}^{1}$ must satisfy the following boundary conditions:

$$
\mathcal{T}_{n}^{1}-\frac{\partial \mathcal{M}_{n t}^{1}}{\partial t}=0, \quad \mathcal{M}_{n n}^{1}=-6 \mathrm{~J}_{\mathrm{f}} \frac{\partial \mathcal{M}_{n t}^{0}}{\partial t}
$$

which differ from the usual ones because of the presence of a boundary layer. The last one involves the universal positive coefficient $\mathrm{J}_{\mathrm{f}}$ given by (56) and coming from the twist boundary layer problem (51).

\section{Analysis of boundary layers near a line of geometrical defects}

The present section is devoted to the study of the boundary layers induced by a "line of defects" localised near the plane $x_{2}=0$ of the plate. Specifically, the goal is to deduce from an asymptotic analysis the effective transmission conditions across the line $x_{2}=0$ that the first two displacement fields $U^{0}$ and $U^{1}$ (or their derivatives) have to satisfy.

\subsection{The assumed type of defects and notations}

The plate is "slightly" modified by geometrical defects in the neighbourhood of the plane $x_{2}=0$, the size of which is of the order of the thickness $h$. These defects can be cracks, voids, geometrical stiffeners, or weakeners which are assumed to be symmetrically arranged with respect to the mid-plane $x_{3}=0$ of the plate. To simplify the presentation we will only consider here the case of geometrical stiffening or weakening like in Figure 4. Specifically, the three-dimensional plate with the geometrical defect has for reference configuration

$$
\Omega_{R}^{d}=\left\{\mathbf{x}:\left(x_{1}, x_{2}\right) \in(-L / 2,+L / 2) \times(-\ell / 2, \ell / 2),\left|x_{3}\right|<H\left(x_{2}\right) / 2\right\},
$$

so that the defect is invariant in the direction $x_{1}$ and its height $H\left(x_{2}\right)$ is equal to the constant $h$ as soon as $\left|x_{2}\right| / h$ is large enough. We assume that those defects can modify locally the distribution of the body forces and the surface forces, but they do not change the properties of symmetry of the fields.
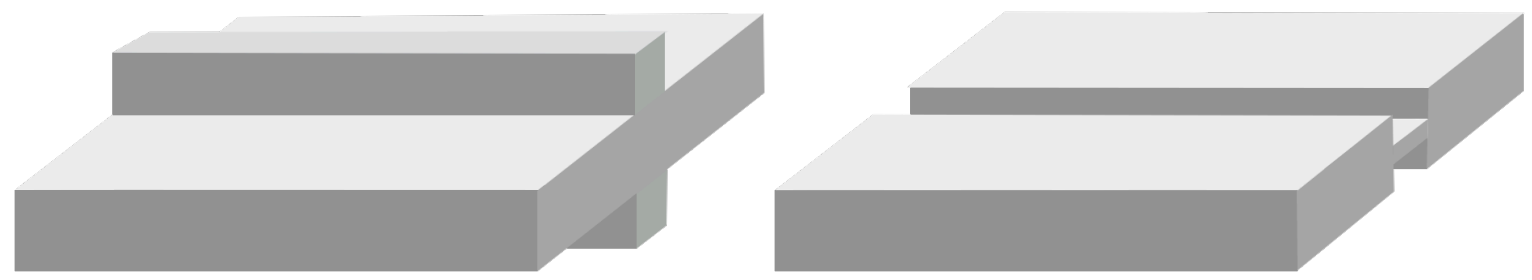

Fig. 4 Examples of defect near the plane $x_{2}=0$ of the plate: (left) geometrical stiffening, (right) geometrical weakening.

Throughout this section $y_{2}=\bar{x}_{2} / \eta$ and hence $\left(y_{2}, y_{3}\right)$ represent the small scale coordinates in the neighbourhood of the defect whereas $\bar{x}_{1}$ is the large scale coordinate. Specifically, the boundary layer problems will be posed on an infinite strip Y of the $\left(y_{2}, y_{3}\right)$ plane, see Figure 5 , whereas $\bar{x}_{1}$ will only play the role of a parameter varying in $I_{1}=(-1 / 2,1 / 2)$. Accordingly, we denote by $\mathbf{y}$ the pair $\left(y_{2}, y_{3}\right)$ in $\mathrm{Y}$. The upper side of the strip $\mathrm{Y}$ is denoted $\partial_{+} \mathrm{Y}$. Since we will have to compare the perturbed cell $\mathrm{Y}$ with the perfect one $\mathrm{Y}_{0}=\mathbb{R} \times(0,1 / 2)$, we denote by $\mathrm{Y} \backslash \mathrm{Y}_{0}$ the part of $\mathrm{Y}$ which is not in $\mathrm{Y}_{0}$, and by $\mathrm{Y}_{0} \backslash \mathrm{Y}$ the part of $\mathrm{Y}_{0}$ which is not in $\mathrm{Y}$ :

$$
\mathrm{Y} \backslash \mathrm{Y}_{0}=\mathrm{Y} \cap\left\{y_{3} \geq 1 / 2\right\}, \quad \mathrm{Y}_{0} \backslash \mathrm{Y}=\mathrm{Y}_{0} \cap \mathrm{Y}^{c} .
$$

Moreover, we introduce the following integral for a function $\varphi$ defined in $\mathrm{Y} \cup \mathrm{Y}_{0}$ :

$$
\int_{\mathrm{Y}-\mathrm{Y}_{0}} \varphi(\mathbf{y}) d \mathbf{y}=\int_{\mathrm{Y} \backslash \mathrm{Y}_{0}} \varphi(\mathbf{y}) d \mathbf{y}-\int_{\mathrm{Y}_{0} \backslash \mathrm{Y}} \varphi(\mathbf{y}) d \mathbf{y} .
$$

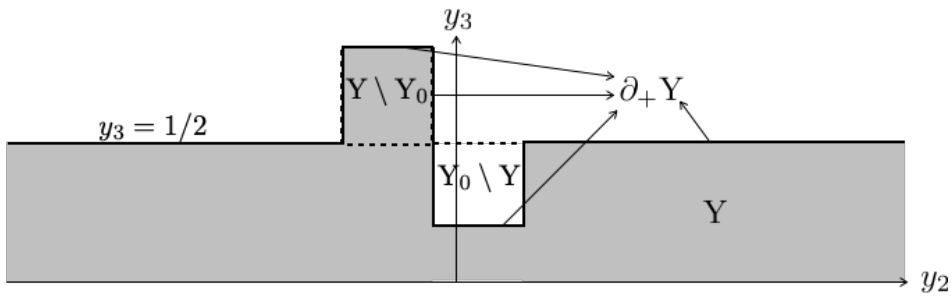

Fig. 5 The infinite strip $\mathrm{Y}$ with the defect which consists here both in a geometrical stiffening and a geometrical weakening. 
Otherwise, the notations are the same as those of Section 4. In particular Lemma 2 still holds.

\subsection{Hypothesis on the form of expansions and matching conditions}

The expansion (14) remains valid far enough from the plane $\bar{x}_{2}=0$ but must be changed in its neighbourhood. Specifically, we suppose that, near the plane $\bar{x}_{2}=0$, the displacement $\mathbf{u}^{\eta}$ admits the following asymptotic expansion with respect to the small parameter $\eta$

$$
\mathbf{u}^{\eta}\left(\overline{\mathbf{x}}, y_{3}\right)=\mathbf{v}^{0}\left(\bar{x}_{1}, \mathbf{y}\right)+\eta \mathbf{v}^{1}\left(\bar{x}_{1}, \mathbf{y}\right)+\eta^{2} \mathbf{v}^{2}\left(\bar{x}_{1}, \mathbf{y}\right)+\eta^{3} \mathbf{v}^{3}\left(\bar{x}_{1}, \mathbf{y}\right)+\cdots,
$$

with $\bar{x}_{1} \in I_{1}$ and $\mathbf{y}=\left(\bar{x}_{2} / \eta, y_{3}\right) \in \mathrm{Y}$. Consequently, strains and stresses admit the same type of asymptotic expansion which a priori starts at order -2

$$
\left\{\begin{array}{l}
\varepsilon^{\eta}\left(\overline{\mathbf{x}}, y_{3}\right)=\eta^{-2} \boldsymbol{\gamma}^{-2}\left(\bar{x}_{1}, \mathbf{y}\right)+\eta^{-1} \boldsymbol{\gamma}^{-1}\left(\bar{x}_{1}, \mathbf{y}\right)+\boldsymbol{\gamma}^{0}\left(\bar{x}_{1}, \mathbf{y}\right)+\eta \boldsymbol{\gamma}^{1}\left(\bar{x}_{1}, \mathbf{y}\right)+\eta^{2} \boldsymbol{\gamma}^{2}\left(\bar{x}_{1}, \mathbf{y}\right)+\cdots \\
\boldsymbol{\sigma}^{\eta}\left(\overline{\mathbf{x}}, y_{3}\right)=\eta^{-2} \boldsymbol{\tau}^{-2}\left(\bar{x}_{1}, \mathbf{y}\right)+\eta^{-1} \boldsymbol{\tau}^{-1}\left(\bar{x}_{1}, \mathbf{y}\right)+\boldsymbol{\tau}^{0}\left(\bar{x}_{1}, \mathbf{y}\right)+\eta \boldsymbol{\tau}^{1}\left(\bar{x}_{1}, \mathbf{y}\right)+\eta^{2} \boldsymbol{\tau}^{2}\left(\bar{x}_{1}, \mathbf{y}\right)+\cdots
\end{array}\right.
$$

For displacements, matching conditions with the expansion (14) read as

$$
\left\{\begin{array}{l}
\mathbf{u}^{0}\left(\bar{x}_{1}, 0 \pm, y_{3}\right)=\lim _{y_{2} \rightarrow \pm \infty} \mathbf{v}^{0}\left(\bar{x}_{1}, y_{2}, y_{3}\right), \\
\mathbf{u}^{1}\left(\bar{x}_{1}, 0 \pm, y_{3}\right)=\lim _{y_{2} \rightarrow \pm \infty}\left(\mathbf{v}^{1}\left(\bar{x}_{1}, y_{2}, y_{3}\right)-y_{2} \mathbf{u}_{, 2}^{0}\left(\bar{x}_{1}, 0 \pm, y_{3}\right)\right) \\
\mathbf{u}^{2}\left(\bar{x}_{1}, 0 \pm, y_{3}\right)=\lim _{y_{2} \rightarrow \pm \infty}\left(\mathbf{v}^{2}\left(\bar{x}_{1}, y_{2}, y_{3}\right)-y_{2} \mathbf{u}_{, 2}^{1}\left(\bar{x}_{1}, 0 \pm, y_{3}\right)-\frac{1}{2} y_{2}^{2} \mathbf{u}_{, 22}^{0}\left(\bar{x}_{1}, 0 \pm, y_{3}\right)\right) .
\end{array}\right.
$$

Let us note that matching conditions must be written on each side of the line of defect and one must assume $a$ priori that the outer expansions obtained in Subsection 2.3 can be discontinuous across $\bar{x}_{2}=0$. The same type of matching conditions hold for stresses, except that they start at order -2 instead of 0 (and hence all the indices must be shifted).

\subsection{Determination of the first three kinematic transmission conditions}

In this subsection we want to obtain the kinematic transmission conditions that $U^{0}, U^{1}, U_{, 2}^{0}$ and $U_{, 2}^{1}$ satisfy across the line $\bar{x}_{2}=0$. Inserting the expansions (60) into (6)-(9) gives for the leading terms the following set of equations

$$
\left\{\begin{array}{lr}
\operatorname{div}_{\mathbf{y}} \boldsymbol{\tau}^{-2}=\mathbf{0} & \text { in } I_{1} \times \mathrm{Y} \\
\boldsymbol{\gamma}^{-2}=\varepsilon_{\mathbf{y}}\left(\mathbf{v}^{0}\right)=-\nu \operatorname{Tr} \boldsymbol{\tau}^{-2} \mathbf{I}+(1+\nu) \boldsymbol{\tau}^{-2} & \text { in } I_{1} \times \mathrm{Y} \\
v_{1}^{0}=v_{2}^{0}=\tau_{33}^{-2}=0 \text { at } y_{3}=0, \quad \boldsymbol{\tau}^{-2} \mathbf{n}=\mathbf{0} & \text { on } I_{1} \times \partial_{+} \mathrm{Y}
\end{array}\right.
$$

Moreover, since $\boldsymbol{\sigma}^{-2}=\mathbf{0}$, the first matching condition for stresses gives $\boldsymbol{\tau}^{-2}\left(\bar{x}_{1}, \pm \infty, y_{3}\right)=0$. Multiplying the equilibrium equation by $\mathbf{v}^{0}$ and integrating over $I_{1} \times \mathrm{Y}$ leads to $\int_{I_{1} \times \mathrm{Y}} \operatorname{div}_{\mathbf{y}} \boldsymbol{\tau}^{-2} \cdot \mathbf{v}^{0} d \bar{x}_{1} d \mathbf{y}=0$. Integrating by parts the integral, using boundary conditions, and matching conditions gives $\int_{I_{1} \times \mathrm{Y}} \boldsymbol{\tau}^{-2} \cdot \varepsilon_{\mathbf{y}}\left(\mathbf{v}^{0}\right) d \bar{x}_{1} d \mathbf{y}=0$. Then, by the constitutive relation between $\boldsymbol{\tau}^{-2}$ and $\varepsilon_{\mathbf{y}}\left(\mathbf{v}^{0}\right)$, we conclude that $\varepsilon_{\mathbf{y}}\left(\mathbf{v}^{0}\right)=\mathbf{0}$. Finally, Lemma 2 gives

$$
\mathbf{v}^{0}\left(\bar{x}_{1}, \mathbf{y}\right)=V^{0}\left(\bar{x}_{1}\right) \mathbf{e}_{3}+\omega^{0}\left(\bar{x}_{1}\right) \mathbf{e}_{1} \wedge \mathbf{y}, \quad \boldsymbol{\tau}^{-2}=\mathbf{0}, \quad \gamma^{-2}=\mathbf{0} \quad \text { in } \quad I_{1} \times \mathrm{Y} .
$$

Moreover, the first of matching conditions (61) combined with (15) gives $\omega^{0}=0$ and $U^{0}\left(\bar{x}_{1}, 0 \pm\right)=V^{0}\left(\bar{x}_{1}\right)$, the continuity of $U^{0}$ at $\bar{x}_{2}=0$ follows which constitutes the first transmission condition

$$
\llbracket U^{0} \rrbracket=0 \quad \text { at } \quad \bar{x}_{2}=0
$$

where the double brackets denote the jump, $\llbracket \rrbracket\left(\bar{x}_{1}\right)=\varphi\left(\bar{x}_{1}, 0+\right)-\varphi\left(\bar{x}_{1}, 0-\right)$. Let us set $\mathbf{v}^{*}\left(\bar{x}_{1}, \mathbf{y}\right)=y_{3} U_{, 1}^{0}\left(\bar{x}_{1}, 0\right) \mathbf{e}_{1}$ and remark that $\varepsilon_{\bar{x}}\left(\mathbf{v}^{0}\right)=\varepsilon_{\mathbf{y}}\left(\mathbf{v}^{*}\right)$. Considering now the next terms of the expansion, one deduces from (6)-(9) that $\boldsymbol{\tau}^{-1}$ and $\mathbf{v}^{1}$ must satisfy

$$
\left\{\begin{array}{lr}
\operatorname{div}_{\mathbf{y}} \boldsymbol{\tau}^{-1}=\mathbf{0} & \text { in } I_{1} \times \mathrm{Y} \\
\varepsilon_{\mathbf{y}}\left(\mathbf{v}^{1}+\mathbf{v}^{*}\right)=-\nu \operatorname{Tr} \boldsymbol{\tau}^{-1} \mathbf{I}+(1+\nu) \boldsymbol{\tau}^{-1} & \text { in } I_{1} \times \mathrm{Y} \\
v_{1}^{1}=v_{2}^{1}=\tau_{33}^{-1}=0 \text { at } y_{3}=0, \quad \boldsymbol{\tau}^{-1} \mathbf{n}=\mathbf{0} & \text { on } I_{1} \times \partial_{+} \mathrm{Y}
\end{array}\right.
$$

Since $\boldsymbol{\sigma}^{-1}=\mathbf{0}$ and since $\mathbf{v}^{*}$ satisfies the boundary condition at $y_{3}=0$, one can follow the same procedure as for $\mathbf{v}^{0}$ and $\boldsymbol{\tau}^{-2}$ to obtain $\int_{I_{1} \times \mathrm{Y}} \boldsymbol{\tau}^{-1} \cdot \varepsilon_{\mathbf{y}}\left(\mathbf{v}^{1}+\mathbf{v}^{*}\right) d \bar{x}_{1} d \mathbf{y}=0$ and hence $\varepsilon_{\mathbf{y}}\left(\mathbf{v}^{1}+\mathbf{v}^{*}\right)=\mathbf{0}$ and $\boldsymbol{\tau}^{-1}=\mathbf{0}$ in $I_{1} \times Y$. 
Therefore, by Lemma $2, \mathbf{v}^{1}+\mathbf{v}^{*}=V^{1}\left(\bar{x}_{1}\right) \mathbf{e}_{3}+\omega^{1}\left(\bar{x}_{1}\right) \mathbf{e}_{1} \wedge \mathbf{y}$. Then, using (15)-(16), the second matching conditions (61) give

$$
-y_{3} U_{, 2}^{0}\left(\bar{x}_{1}, 0 \pm\right) \mathbf{e}_{2}+U^{1}\left(\bar{x}_{1}, 0 \pm\right) \mathbf{e}_{3}=-y_{3} \omega^{1}\left(\bar{x}_{1}\right) \mathbf{e}_{2}+V^{1}\left(\bar{x}_{1}\right) \mathbf{e}_{3}+\lim _{y_{2} \rightarrow \pm \infty} y_{2}\left(\omega^{1}\left(\bar{x}_{1}\right)-U_{, 2}^{0}\left(\bar{x}_{1}, 0 \pm\right)\right) \mathbf{e}_{3}
$$

and hence are satisfied if and only if $V^{1}\left(\bar{x}_{1}\right)=U^{1}\left(\bar{x}_{1}, 0 \pm\right)$ and $\omega^{1}\left(\bar{x}_{1}\right)=U_{, 2}^{0}\left(\bar{x}_{1}, 0 \pm\right)$. One deduces the continuity of $U^{1}$ and $U_{, 2}^{0}$ at $\bar{x}_{2}=0$, that constitute two other transmission conditions

$$
\llbracket U_{, 2}^{0} \rrbracket=0 \quad \text { and } \quad \llbracket U^{1} \rrbracket=0 \quad \text { at } \quad \bar{x}_{2}=0 \text {. }
$$

Furthermore, $\mathbf{v}^{1}$ reads

$$
\mathbf{v}^{1}\left(\bar{x}_{1}, \mathbf{y}\right)=-y_{3} U_{, \alpha}^{0}\left(\bar{x}_{1}, 0\right) \mathbf{e}_{\alpha}+\left(U^{1}\left(\bar{x}_{1}, 0\right)+y_{2} U_{, 2}^{0}\left(\bar{x}_{1}, 0\right)\right) \mathbf{e}_{3}
$$

It remains to find the transmission condition for $U_{, 2}^{1}$. It will be given by the inner problem at next order which gives also the first static transmission condition.

5.4 Determination of the last kinematic and the first static transmission conditions

Using the previous results, one deduces from (6)-(9) that $\boldsymbol{\tau}^{0}$ and $\mathbf{v}^{2}$ must satisfy

$$
\left\{\begin{array}{lr}
\operatorname{div}_{\mathbf{y}} \boldsymbol{\tau}^{0}=\mathbf{0} & \text { in } I_{1} \times \mathrm{Y} \\
\varepsilon_{\mathbf{y}}\left(\mathbf{v}^{2}\right)+\varepsilon_{\bar{x}}\left(\mathbf{v}^{1}\right)=-\nu \operatorname{Tr} \boldsymbol{\tau}^{0} \mathbf{I}+(1+\nu) \boldsymbol{\tau}^{0} & \text { in } I_{1} \times \mathrm{Y} \\
v_{1}^{2}=v_{2}^{2}=\tau_{33}^{0}=0 \text { at } y_{3}=0, \quad \boldsymbol{\tau}^{0} \mathbf{n}=\mathbf{0} & \text { on } I_{1} \times \partial_{+} \mathrm{Y}
\end{array}\right.
$$

and the condition at infinity for $\tau^{0}$ which is given by the matching condition between $\boldsymbol{\sigma}^{0}$ and $\boldsymbol{\tau}^{0}$, i.e. ,

$$
\lim _{y_{2} \rightarrow \pm \infty} \boldsymbol{\tau}^{0}\left(\bar{x}_{1}, y_{2}, y_{3}\right)=\boldsymbol{\sigma}^{0}\left(\bar{x}_{1}, 0 \pm, y_{3}\right)
$$

Since rigid motions of the form $\mathbf{v}\left(\bar{x}_{1}, \mathbf{y}\right)=V\left(\bar{x}_{1}\right) \mathbf{e}_{3}+\omega\left(\bar{x}_{1}\right) \mathbf{e}_{1} \wedge \mathbf{y}$ are still allowed by virtue of Lemma 2 , the loading at infinity given by $\boldsymbol{\sigma}^{0}$ must satisfy conditions in order that this problem for $\mathbf{v}^{2}$ and $\boldsymbol{\tau}^{0}$ admits at least one solution. Specifically, the $\mathbf{e}_{3}$ component of the resultant force and the $\mathbf{e}_{1}$ component of the resultant moment of the external forces must vanish. That leads to the following two conditions:

$$
\int_{\partial \mathrm{Y}} \boldsymbol{\tau}^{0} \mathbf{n} \cdot \mathbf{e}_{3} d s=0, \quad \int_{\partial \mathrm{Y}}\left(\mathbf{y} \wedge \boldsymbol{\tau}^{0} \mathbf{n}\right) \cdot \mathbf{e}_{1} d s=0
$$

where $\partial \mathrm{Y}$ denotes the boundary of $\mathrm{Y}$ including the sections at infinity $\{ \pm \infty\} \times(0,1 / 2)$. By virtue of the boundary conditions satisfied by $\tau^{0}$ and the fact that $\sigma_{\alpha 3}^{0}=0$, the first equality is automatically satisfied whereas the second is reduced to

$$
\int_{0}^{1 / 2} y_{3} \sigma_{22}^{0}\left(\bar{x}_{1}, 0+, y_{3}\right) d y_{3}=\int_{0}^{1 / 2} y_{3} \sigma_{22}^{0}\left(\bar{x}_{1}, 0-, y_{3}\right) d y_{3} .
$$

Comparing with (10), this condition requires that the normal bending moment $\mathcal{M}_{22}^{0}$ be continuous, namely, the first static transmission condition

$$
\llbracket \mathcal{M}_{22}^{0} \rrbracket=0 \quad \text { at } \quad \bar{x}_{2}=0 .
$$

In terms of the derivatives of $U^{0}$, using (20), (64) implies that $U_{, 22}^{0}$ is continuous at $\bar{x}_{2}=0$ and hence $U^{0}$ and all its derivatives up to the second order are continuous. Consequently, all the components of the stress tensor $\sigma^{0}$ and the moment tensor $\mathcal{M}^{0}$ are continuous. For the shear force $\mathcal{T}^{0}$, its tangential component $\mathcal{T}_{1}^{0}$ is continuous but the jump condition for its normal component $\mathcal{T}_{2}^{0}$ remains to be determined.

Assuming that the condition (64) is satisfied, the problem (62)-(63) admits a solution which is defined up to an admissible rigid motion. Using the linearity and after tedious calculations which are not reproduced here, the solution eventually reads as follows

$$
\left\{\begin{array}{l}
v_{1}^{2}=-U_{, 12}^{0} y_{2} y_{3}-U_{, 1}^{1} y_{3}+U_{, 12}^{0} \mathbf{W}^{\mathrm{d}}(\mathbf{y}) \\
v_{2}^{2}=-U_{, 22}^{0} y_{2} y_{3}+\left(U_{, 22}^{0}+\nu U_{, 11}^{0}\right) V_{2}^{d}(\mathbf{y})-\omega^{2}\left(\bar{x}_{1}\right) y_{3} \\
v_{3}^{2}=U_{, 22}^{0} \frac{y_{2}^{2}}{2}+\left(U_{, 11}^{0}+U_{, 22}^{0}\right) \frac{\nu y_{3}^{2}}{2(1-\nu)}+\left(U_{, 22}^{0}+\nu U_{, 11}^{0}\right) V_{3}^{d}(\mathbf{y})+V^{2}\left(\bar{x}_{1}\right)+\omega^{2}\left(\bar{x}_{1}\right) y_{2}
\end{array}\right.
$$




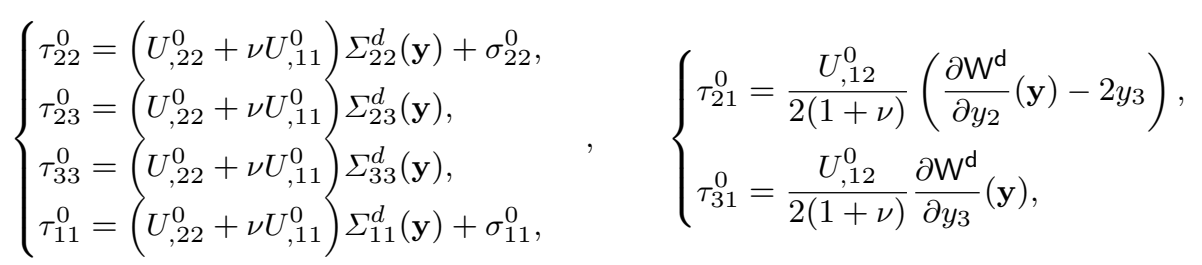

where second derivatives of $U^{0}, U_{, 2}^{1}$ and $\sigma^{0}$ are evaluated at $\bar{x}_{2}=0$. All these quantities are well defined by virtue of the already obtained continuity properties. In (65), $V^{2}$ and $\omega^{2}$ denote a translation and a rotation, left arbitrary. In (65) and (66), the pair of displacement and stress fields $\left(\mathbf{V}^{\mathbf{d}}, \boldsymbol{\Sigma}^{\mathbf{d}}\right)$ is solution of the following plane strain problem posed in the strip $\mathrm{Y}$, called the bending boundary layer problem near the defect:

$$
\left\{\begin{array}{lr}
\operatorname{div}_{\mathbf{y}} \boldsymbol{\Sigma}^{\mathbf{d}}=\mathbf{0} & \text { in } \mathrm{Y}, \\
\varepsilon_{\mathbf{y}}\left(\mathbf{V}^{\mathbf{d}}\right)=-\nu \operatorname{Tr} \boldsymbol{\Sigma}^{\mathbf{d}} \mathbf{I}+(1+\nu) \boldsymbol{\Sigma}^{\mathbf{d}} & \text { in } \mathrm{Y}, \\
V_{2}^{d}=\Sigma_{33}^{d}=0 & \text { at } y_{3}=0 \\
\boldsymbol{\Sigma}^{\mathbf{d}} \mathbf{n}=\frac{y_{3} n_{2}}{1-\nu^{2}} \mathbf{e}_{2} & \text { on } \partial_{+} \mathrm{Y}, \\
\lim _{y_{2} \rightarrow \pm \infty} \boldsymbol{\Sigma}^{\mathbf{d}}(\mathbf{y})=\mathbf{0}, &
\end{array}\right.
$$

whereas the displacement field $\mathrm{W}^{\mathrm{d}}$ is the unique solution of the following out-of-plane problem posed in $\mathrm{Y}$, called the twist boundary layer problem near the defect:

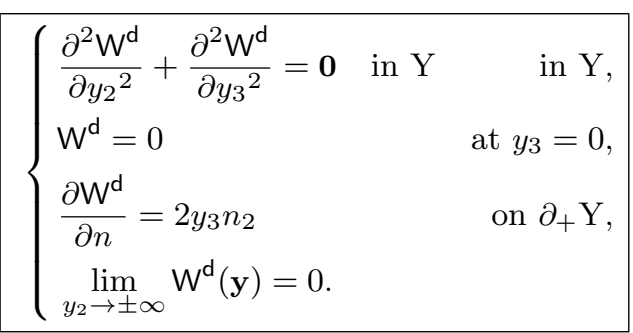

These two boundary layer problems will be studied in details in the next subsections. However, the determination of the last kinematic transmission condition requires some preliminary results concerning the bending boundary layer problem near the defect.

Because $\boldsymbol{\Sigma}^{\mathbf{d}}$ tends to $\mathbf{0}$ when $\left|y_{2}\right|$ tends to infinity, so does $\varepsilon_{\mathbf{y}}\left(\mathbf{V}^{\mathbf{d}}\right)$. Consequently, since $V_{2}^{d}=0$ on $y_{3}=0$, by virtue of Lemma 2, there exists four constants $\mathrm{K}_{\mathrm{d}}^{+}, \mathrm{K}_{\mathrm{d}}^{-}, \mathrm{C}_{\mathrm{d}}^{+}$and $\mathrm{C}_{\mathrm{d}}^{-}$(depending only on the geometry of the defect and on the Poisson ratio) such that

$$
\lim _{y_{2} \rightarrow+\infty}\left(\mathbf{V}^{\mathbf{d}}(\mathbf{y})-\mathrm{C}_{\mathrm{d}}^{+} \mathbf{e}_{1} \wedge \mathbf{y}\right)=\mathrm{K}_{\mathrm{d}}^{+} \mathbf{e}_{3}, \quad \lim _{y_{2} \rightarrow-\infty}\left(\mathbf{V}^{\mathbf{d}}(\mathbf{y})-\mathrm{C}_{\mathrm{d}}^{-} \mathbf{e}_{1} \wedge \mathbf{y}\right)=\mathrm{K}_{\mathrm{d}}^{-} \mathbf{e}_{3}
$$

Since $\mathbf{V}^{\mathbf{d}}$ is defined up to an admissible rigid displacement, only the difference between the limits at infinity are fixed. Accordingly, we set

$$
\mathrm{C}_{\mathrm{d}}=\mathrm{C}_{\mathrm{d}}^{-}-\mathrm{C}_{\mathrm{d}}^{+}, \quad \mathrm{K}_{\mathrm{d}}=\mathrm{K}_{\mathrm{d}}^{+}-\mathrm{K}_{\mathrm{d}}^{-} \text {, }
$$

where the definition of $C_{d}$ is chosen so that $C_{d}$ be positive in the case of a reinforcement (see Section 5.7). Using (16), (18), (65) and (69), the third matching condition (61) for $v_{2}^{2}$ gives

$$
U_{, 2}^{1}\left(\bar{x}_{1}, 0 \pm\right)=\mathrm{C}_{\mathrm{d}}^{ \pm}\left(U_{, 22}^{0}\left(\bar{x}_{1}, 0\right)+\nu U_{, 11}^{0}\left(\bar{x}_{1}, 0\right)\right)+\omega^{2}\left(\bar{x}_{1}\right)
$$

and hence the last kinematic transmission condition

$$
\llbracket U_{, 2}^{1} \rrbracket=-C_{\mathrm{d}}\left(U_{, 22}^{0}+\nu U_{, 11}^{0}\right) \quad \text { at } \quad \bar{x}_{2}=0 .
$$

The above jump condition can be also read in term of the bending moment $\mathcal{M}_{22}^{0}: \mathrm{D} \llbracket U_{, 2}^{1} \rrbracket=-\mathrm{C}_{\mathrm{d}} \mathcal{M}_{22}^{0}$. The third matching condition (61) for $v_{1}^{2}$ is automatically satisfied, essentially because $\lim _{y_{2} \rightarrow \pm \infty} \mathrm{W}^{\mathrm{d}}(y)=0$. Furthermore the third matching condition (61) for $v_{3}^{2}$ gives a first transmission condition for $U^{2}, \llbracket U^{2} \rrbracket=\mathrm{K}_{\mathrm{d}}\left(U_{, 22}^{0}+\nu U_{, 11}^{0}\right)$, that will be not used in the sequel. 


\subsection{Determination of two other static transmission conditions}

Let us consider the next inner problem for $\tau^{1}$ and $\mathbf{v}^{3}$. The stress field $\tau^{1}$ must satisfy

$$
\left\{\begin{array}{lr}
\operatorname{div}_{\mathbf{y}} \boldsymbol{\tau}^{1}+\boldsymbol{d i v}_{\bar{x}} \boldsymbol{\tau}^{0}=\mathbf{0} & \text { in } I_{1} \times \mathrm{Y}, \\
\tau_{33}^{1}=0 \text { at } y_{3}=0, \quad \boldsymbol{\tau}^{1} \mathbf{n}=\mathbf{0} & \text { on } I_{1} \times \partial_{+} \mathrm{Y}, \\
\lim _{y_{2} \rightarrow \pm \infty}\left(\boldsymbol{\tau}^{1}\left(\bar{x}_{1}, y_{2}, y_{3}\right)-y_{2} \boldsymbol{\sigma}_{, 2}^{0}\left(\bar{x}_{1}, 0 \pm, y_{3}\right)\right)=\boldsymbol{\sigma}^{1}\left(\bar{x}_{1}, 0 \pm, y_{3}\right) . &
\end{array}\right.
$$

In order that a solution exists, the $\mathbf{e}_{3}$ component of the resultant force and the $\mathbf{e}_{1}$ component of the resultant moment of the external forces must vanish. These equilibrium conditions will give us two static transmission conditions.

The former condition is obtained by integrating the third component of the equilibrium equation over Y. Specifically, that leads to

$$
0=\int_{\mathrm{Y}} 2\left(\operatorname{div}_{\mathbf{y}} \boldsymbol{\tau}^{1}+\operatorname{div}_{\bar{x}} \boldsymbol{\tau}^{0}\right) \cdot \mathbf{e}_{3} d \mathbf{y}=\int_{0}^{1 / 2} 2\left(\tau_{32}^{1}\left(\bar{x}_{1},+\infty, y_{3}\right)-\tau_{32}^{1}\left(\bar{x}_{1},-\infty, y_{3}\right)\right) d y_{3}+\int_{\mathrm{Y}} 2 \frac{\partial \tau_{31}^{0}}{\partial \bar{x}_{1}}\left(\bar{x}_{1}, \mathbf{y}\right) d \mathbf{y}
$$

Using the condition at infinity with $\sigma_{32}^{0}=0$ and (66), one gets

$$
0=\int_{0}^{1 / 2} 2\left(\sigma_{32}^{1}\left(\bar{x}_{1}, 0+, y_{3}\right)-\sigma_{32}^{1}\left(\bar{x}_{1}, 0-, y_{3}\right)\right) d y_{3}+\frac{U_{, 211}^{0}\left(\bar{x}_{1}, 0\right)}{1+\nu} \int_{\mathrm{Y}} \frac{\partial \mathbf{W}^{\mathrm{d}}}{\partial y_{3}}(\mathbf{y}) d \mathbf{y} .
$$

The first integral in (72) is the jump of the shear force $\mathcal{T}_{2}^{0}$. Let us show that it vanishes because $\int_{\mathrm{Y}} \frac{\partial \mathrm{W}^{\mathrm{d}}}{\partial y_{3}} d \mathbf{y}=0$. Indeed, by virtue of (68), one has

$$
0=\int_{\mathrm{Y}} y_{3} \Delta \mathrm{W}^{\mathrm{d}} d \mathbf{y}=-\int_{\mathrm{Y}} \frac{\partial \mathrm{W}^{\mathrm{d}}}{\partial y_{3}} d \mathbf{y}+\int_{\partial \mathrm{Y}} y_{3} \frac{\partial \mathrm{W}^{\mathrm{d}}}{\partial n} d s=-\int_{\mathrm{Y}} \frac{\partial \mathrm{W}^{\mathrm{d}}}{\partial y_{3}} d \mathbf{y}+\int_{\partial_{+} \mathrm{Y}} 2 y_{3}^{2} n_{2} d s
$$

But since

$$
0=\int_{\mathrm{Y}} \frac{\partial\left(y_{3}^{2}\right)}{\partial y_{2}} d \mathbf{y}=\int_{\partial_{+} \mathrm{Y}} y_{3}^{2} n_{2} d s
$$

the result follows. Hence we have obtained the second static transmission condition

$$
\llbracket \mathcal{T}_{2}^{0} \rrbracket=0 \quad \text { at } \quad \bar{x}_{2}=0
$$

which can be also read as $\llbracket \mathcal{T}_{2}^{0}-\mathcal{M}_{21,1}^{0} \rrbracket=0$ owing to the continuity of $U^{0}$ and $U_{, 2}^{0}$. Moreover (73) implies that $U_{, 222}^{0}$ and hence all the third derivatives of $U^{0}$ are continuous at $\bar{x}_{2}=0$.

Let us consider now the condition of vanishing of the $\mathbf{e}_{1}$ component of the resultant moment of the external forces. Multiplying the equilibrium equation in (71) by $2 \mathbf{e}_{1} \wedge \mathbf{y}$ and integrating, at a given $\bar{x}_{1}$, with respect to $\mathbf{y}$ over $\mathrm{Y}_{\zeta}=\mathrm{Y} \cap\left\{\left|y_{2}\right|<\zeta\right\}$ leads to

$$
0=\int_{\left\{\bar{x}_{1}\right\} \times \mathrm{Y}_{\zeta}} 2 \operatorname{div}_{\mathbf{y}} \boldsymbol{\tau}^{1} \cdot\left(\mathbf{e}_{1} \wedge \mathbf{y}\right) d \mathbf{y}+\frac{U_{, 211}^{0}}{1+\nu} \int_{\mathrm{Y}_{\zeta}}\left(y_{2} \frac{\partial \mathbf{W}^{\mathrm{d}}}{\partial y_{3}}-y_{3} \frac{\partial \mathbf{W}^{\mathrm{d}}}{\partial y_{2}}+2 y_{3}^{2}\right) d \mathbf{y},
$$

where we have used the expression (66) for $\tau^{0}$. Since $\zeta$ is destined to tend to infinity, we assume from now that $\zeta$ is sufficiently large so that the geometric defect is entirely included in $\mathrm{Y}_{\zeta}$. Integrating by parts the first integral, using the expression (20) of $\mathcal{M}_{21}^{0}$ and the boundary conditions give

$$
\begin{aligned}
\int_{0}^{1 / 2} 2 y_{3}\left(\tau_{22}^{1}\left(\bar{x}_{1}, \zeta, y_{3}\right)-\tau_{22}^{1}\left(\bar{x}_{1},-\zeta, y_{3}\right)\right) d y_{3} & =2 \zeta \int_{0}^{1 / 2}\left(\tau_{32}^{1}\left(\bar{x}_{1}, \zeta, y_{3}\right)+\tau_{32}^{1}\left(\bar{x}_{1},-\zeta, y_{3}\right)\right) d y_{3} \\
& +12 \mathcal{M}_{21,1}^{0}\left(\bar{x}_{1}, 0\right) \int_{\mathrm{Y}_{\zeta}}\left(y_{2} \frac{\partial \mathbf{W}^{\mathrm{d}}}{\partial y_{3}}-y_{3} \frac{\partial \mathbf{W}^{\mathrm{d}}}{\partial y_{2}}+2 y_{3}^{2}\right) d \mathbf{y} .
\end{aligned}
$$

After multiplying by $2 y_{3}$ and integrating with respect to $y_{3}$ the condition at infinity in (71), one gets for the 22 and 32 components

$$
\lim _{\zeta \rightarrow \pm \infty}\left(\int_{0}^{1 / 2} 2 y_{3} \tau_{22}^{1}\left(\bar{x}_{1}, \pm \zeta, y_{3}\right) d y_{3} \pm \zeta \mathcal{M}_{22,2}^{0}\left(\bar{x}_{1}, 0\right)\right)=-\mathcal{M}_{22}^{1}\left(\bar{x}_{1}, 0 \pm\right)
$$


and

$$
\lim _{\zeta \rightarrow \pm \infty} \int_{0}^{1 / 2} 2 \tau_{32}^{1}\left(\bar{x}_{1}, \pm \zeta, y_{3}\right) d y_{3}=\mathcal{T}_{2}^{0}\left(\bar{x}_{1}, 0\right) .
$$

The equilibrium equation (11) at order 0 gives $\mathcal{T}_{2}^{0}+\mathcal{M}_{21,1}^{0}+\mathcal{M}_{22,2}^{0}=0$ which can be used at $\bar{x}_{2}=0$ because all quantities are continuous. Since all the convergences above are exponential with respect to $\zeta$, after passing to the limit into (74) when $\zeta$ goes to infinity, one finally obtains the third static transmission condition

$$
\llbracket \mathcal{M}_{22}^{1} \rrbracket=-6 \mathrm{~J}_{\mathrm{d}} \mathcal{M}_{21,1}^{0} \quad \text { at } \quad \bar{x}_{2}=0
$$

where $J_{d}$ is the coefficient defined by

$$
\mathrm{J}_{\mathrm{d}}=4 \int_{\mathrm{Y}-\mathrm{Y}_{0}} y_{3}^{2} d \mathbf{y}+2 \int_{\mathrm{Y}}\left(y_{2} \frac{\partial \mathrm{W}^{\mathrm{d}}}{\partial y_{3}}-y_{3} \frac{\partial \mathrm{W}^{\mathrm{d}}}{\partial y_{2}}\right) d \mathbf{y}
$$

the factor 6 being introduced so that $J_{d}$ has a physical interpretation. To obtain $(75)$ we used also the relationship $24 \int_{\mathrm{Y}_{\zeta}} y_{3}^{2} d \mathbf{y}=2 \zeta+24 \int_{\mathrm{Y}-Y_{0}} y_{3}^{2} d \mathbf{y}$ for $\zeta$ large enough.

5.6 Determination of the last static transmission condition

The last static transmission condition is obtained from the equilibrium equation at order 0 . Since the given body forces and surface forces appear at this order, one must make an assumption on their regularity in the neighbourhood of $\bar{x}_{2}=0$ and on how they are modified by the defect. Accordingly, we assume that body forces depend smoothly on $\bar{x}_{2}$ and hence that, for $\left(\bar{x}_{1}, y\right) \in I_{1} \times \mathrm{Y}$,

$$
f\left(\bar{x}_{1}, \eta y_{2}, y_{3}\right)=f_{0}\left(\bar{x}_{1}, y_{3}\right)+\text { higher order terms. }
$$

As far as surface forces are concerned, to simplify the presentation, we assume that they vanish in the neighbourhood of $\bar{x}_{2}=0$. Accordingly, the equilibrium equation at order 0 and the associated boundary or matching conditions for stresses read as

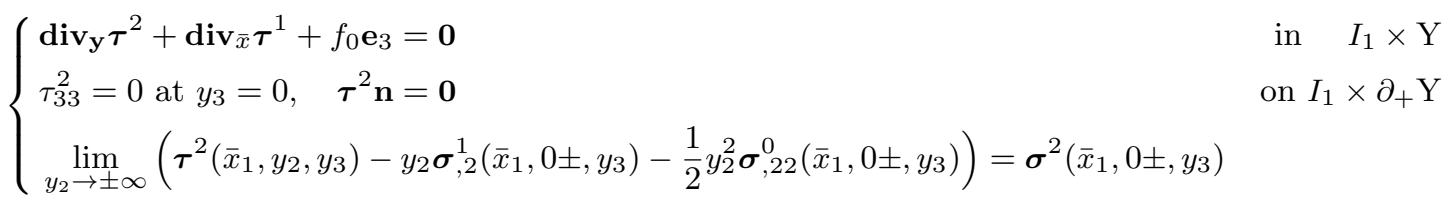

Integrating the third component of the equilibrium equation over $\mathrm{Y}_{\zeta}$ and using boundary conditions leads to

$$
\begin{aligned}
0 & =\int_{\mathrm{Y}_{\zeta}} 2\left(\operatorname{div}_{\mathbf{y}} \boldsymbol{\tau}^{2}+\operatorname{div}_{\bar{x}} \boldsymbol{\tau}^{1}+f_{0} \mathbf{e}_{3}\right) \cdot \mathbf{e}_{3} d \mathbf{y} \\
& =\int_{0}^{1 / 2} 2\left(\tau_{32}^{2}\left(\bar{x}_{1},+\zeta, y_{3}\right)-\tau_{32}^{2}\left(\bar{x}_{1},-\zeta, y_{3}\right)\right) d y_{3}+\int_{\mathrm{Y}_{\zeta}} 2\left(\frac{\partial \tau_{31}^{1}}{\partial \bar{x}_{1}}\left(\bar{x}_{1}, \mathbf{y}\right)+f_{0}\left(y_{3}\right)\right) d \mathbf{y} .
\end{aligned}
$$

The matching condition with (10), (17) and (19) give

$$
\lim _{\zeta \rightarrow \pm \infty}\left(2 \int_{0}^{1 / 2} \tau_{32}^{2}\left(\bar{x}_{1}, \pm \zeta, y_{3}\right) d y_{3} \mp \zeta \mathcal{T}_{2,2}^{0}\left(\bar{x}_{1}, 0\right)\right)=\mathcal{T}_{2}^{1}\left(\bar{x}_{1}, 0 \pm\right)
$$

where we used also the continuity of $\mathcal{T}_{2,2}^{0}$ at $\bar{x}_{2}=0$ by virtue of the equilibrium equation

$$
\mathcal{T}_{2,2}^{0}-\mathcal{M}_{12,12}^{0}-\mathcal{M}_{11,11}^{0}+\int_{0}^{1 / 2} 2 f_{0}\left(\bar{x}_{1}, y_{3}\right) d y_{3}=0
$$

and the assumption on the external forces on the neighbourhood of $\bar{x}_{2}=0$. Therefore (76) becomes

$$
0=\llbracket \mathcal{T}_{2}^{1} \rrbracket+2 \lim _{\zeta \rightarrow \infty}\left(\zeta\left(\mathcal{M}_{12,12}^{0}+\mathcal{M}_{11,11}^{0}\right)+\int_{\mathrm{Y}_{\zeta}} \frac{\partial \tau_{31}^{1}}{\partial \bar{x}_{1}} d \mathbf{y}\right)+2 \int_{\mathrm{Y}-\mathrm{Y}_{0}} f_{0} d \mathbf{y}
$$


where we used (59) to decompose $\int_{\mathbf{Y}_{\zeta}} f_{0} d \mathbf{y}$. It remains to evaluate $\int_{\mathbf{Y}_{\zeta}} \frac{\partial \tau_{31}^{1}}{\partial \bar{x}_{1}} d \mathbf{y}$. Starting from the $\mathbf{e}_{1}$-component of the equilibrium equation (71), once it is multiplied by $2 y_{3}$ and integrated over $\mathrm{Y}_{\zeta}$ one gets

$$
0=\int_{\mathbf{Y}_{\zeta}}\left(2 y_{3} \frac{\partial \tau_{13}^{1}}{\partial y_{3}}+2 y_{3} \frac{\partial \tau_{12}^{1}}{\partial y_{2}}\right) d \mathbf{y}+\int_{\mathbf{Y}_{\zeta}} 2 y_{3} \frac{\partial \tau_{11}^{0}}{\partial \bar{x}_{1}} d \mathbf{y}
$$

Integrating by parts the first term, using the boundary conditions $\tau_{1 i}^{1} n_{i}=0$ on $\partial_{+} \mathrm{Y}$ to simplify the first two terms and using (17) and (66) for the third term leads to

$$
\int_{\mathbf{Y}_{\zeta}} 2 \tau_{13}^{1}\left(\bar{x}_{1}, \mathbf{y}\right) d \mathbf{y}=\int_{0}^{1 / 2} 2 y_{3}\left(\tau_{12}^{1}\left(\bar{x}_{1}, \zeta, y_{3}\right)-\tau_{12}^{1}\left(\bar{x}_{1},-\zeta, y_{3}\right)\right) d y_{3}+\int_{\mathbf{Y}_{\zeta}} 2 y_{3} \frac{\partial \tau_{11}^{0}}{\partial \bar{x}_{1}} d \mathbf{y}
$$

From the matching condition $\lim _{y_{2} \rightarrow \pm \infty}\left(\tau_{12}^{1}\left(\bar{x}_{1}, \mathbf{y}\right)-y_{2} \sigma_{12,2}^{0}\left(0, \bar{x}_{1}, y_{3}\right)\right)=\sigma_{12}^{1}\left(\bar{x}_{1}, 0 \pm, y_{3}\right)$, one gets

$$
\left.\lim _{\zeta \rightarrow \infty} \int_{0}^{1 / 2} 2 y_{3}\left(\tau_{12}^{1}\left(\bar{x}_{1}, \zeta, y_{3}\right)-\tau_{12}^{1}\left(\bar{x}_{1},-\zeta, y_{3}\right)\right) d y_{3}+2 \zeta \mathcal{M}_{12,2}^{0}\left(\bar{x}_{1}, 0\right)\right)=-\llbracket \mathcal{M}_{12}^{1} \rrbracket\left(\bar{x}_{1}, 0\right) .
$$

The expression (66) for $\tau_{11}^{0}$ with $\sigma_{11}^{0}=-12 y_{3} \mathcal{M}_{11}^{0}$ and $\mathcal{M}_{22}^{0}=\mathrm{D}\left(U_{, 22}^{0}+\nu U_{, 11}^{0}\right)$ give

$$
\int_{\mathbf{Y}_{\zeta}} 2 y_{3} \frac{\partial \tau_{11}^{0}}{\partial \bar{x}_{1}} d \mathbf{y}=-2 \zeta \mathcal{M}_{11,1}^{0}-24 \mathcal{M}_{11,1}^{0} \int_{\mathbf{Y}_{-\mathbf{Y}_{0}}} y_{3}^{2} d \mathbf{y}+\frac{1}{\mathrm{D}} \mathcal{M}_{22,1}^{0} \int_{\mathrm{Y}_{\zeta}} 2 y_{3} \Sigma_{11}^{d}(\mathbf{y}) d \mathbf{y}
$$

where we used (59) to decompose $\int_{\mathrm{Y}_{\zeta}} y_{3}^{2} d \mathbf{y}$. Inserting the three previous relations into (77) gives the last static transmission condition

$$
\llbracket \mathcal{T}_{2}^{1}-\mathcal{M}_{21,1}^{1} \rrbracket=24 \mathcal{M}_{11,11}^{0} \int_{\mathrm{Y}-\mathrm{Y}_{0}} y_{3}^{2} d \mathbf{y}-\frac{2}{\mathrm{D}} \mathcal{M}_{22,11}^{0} \int_{\mathrm{Y}} y_{3} \Sigma_{11}^{d}(\mathbf{y}) d \mathbf{y}-2 \int_{\mathrm{Y}-\mathrm{Y}_{0}} f_{0} d \mathbf{y}
$$

The right hand side above can be simplified by virtue of the following equality

$$
\int_{\mathbf{Y}} y_{3}\left(\Sigma_{22}^{d}(\mathbf{y})+\Sigma_{33}^{d}(\mathbf{y})\right) d \mathbf{y}=\int_{\mathbf{Y}-\mathbf{Y}_{0}} \frac{y_{3}^{2}}{1-\nu^{2}} d \mathbf{y} .
$$

To prove (79) one uses the plane strain problem (67). Specifically, one gets for $\zeta$ large enough

$$
0=\int_{\mathbf{Y}_{\zeta}} \operatorname{div}_{\mathbf{y}} \boldsymbol{\Sigma}^{\mathbf{d}} \cdot\left(y_{2} y_{3} \mathbf{e}_{2}+\frac{1}{2}\left(y_{3}^{2}-y_{2}^{2}\right) \mathbf{e}_{3}\right) d \mathbf{y}=-\int_{\mathrm{Y}_{\zeta}} y_{3}\left(\Sigma_{22}^{d}+\Sigma_{33}^{d}\right) d \mathbf{y}+\int_{\partial_{+} \mathrm{Y}_{\zeta}} \frac{y_{3}^{2}}{1-\nu^{2}} y_{2} n_{1} d s+C_{\zeta},
$$

where $\lim _{\zeta \rightarrow \infty} C_{\zeta}=0$ and $\partial_{+} \mathrm{Y}_{\zeta}=\partial_{+} \mathrm{Y} \cap\left\{\left|y_{2}\right|<\zeta\right\}$. Hence

$$
\int_{\mathrm{Y}} y_{3}\left(\Sigma_{22}^{d}+\Sigma_{33}^{d}\right) d \mathbf{y}=\lim _{\zeta \rightarrow+\infty}\left(\int_{\partial_{+} \mathrm{Y}_{\zeta}} \frac{y_{3}^{2} y_{2} n_{1}}{1-\nu^{2}} d s\right)=\frac{1}{1-\nu^{2}} \lim _{\zeta \rightarrow+\infty}\left(\int_{\mathrm{Y}_{\zeta}} y_{3}^{2} d \mathbf{y}-\frac{\zeta}{12}\right)=\int_{\mathrm{Y}-\mathrm{Y}_{0}} \frac{y_{3}^{2}}{1-\nu^{2}} d \mathbf{y} .
$$

Inserting (79) with $\Sigma_{11}^{d}=\nu\left(\Sigma_{22}^{d}+\Sigma_{33}^{d}\right)$ into (78) gives the final form of the last static transmission condition

$$
\llbracket \mathcal{T}_{2}^{1}-\mathcal{M}_{21,1}^{1} \rrbracket=I_{\mathrm{d}} U_{, 1111}^{0}-\mathcal{F}_{0}^{0} \quad \text { at } \quad \bar{x}_{2}=0,
$$

where $I_{d}$ is the coefficient and $\mathcal{F}_{0}^{0}\left(\bar{x}_{1}\right)$ the density of line forces given by

$$
\mathrm{I}_{\mathrm{d}}=2 \int_{\mathrm{Y}-\mathrm{Y}_{0}} y_{3}^{2} d \mathbf{y}, \quad \mathcal{F}_{0}^{0}\left(\bar{x}_{1}\right)=2 \int_{\mathrm{Y}-\mathrm{Y}_{0}} f_{0}\left(\bar{x}_{1}, y_{3}\right) d \mathbf{y} .
$$

One can summarise the results obtained in the present section by the following proposition: 
Proposition 3 Across the line $\bar{x}_{2}=0$ where the defect is located, the displacement $U^{0}$, the shear force vector $\mathcal{T}^{0}$ and the bending moment tensor $\mathcal{M}^{0}$ must satisfy the usual continuity conditions

$$
\llbracket U^{0} \rrbracket=0, \quad \llbracket \frac{\partial U^{0}}{\partial \bar{x}_{2}} \rrbracket=0, \quad \llbracket \mathcal{T}_{2}^{0}-\frac{\partial \mathcal{M}_{21}^{0}}{\partial \bar{x}_{1}} \rrbracket=0, \quad \llbracket \mathcal{M}_{22}^{0} \rrbracket=0 .
$$

The displacement $U^{1}$, the shear force vector $\mathcal{T}^{1}$ and the bending moment tensor $\mathcal{M}^{1}$ must satisfy the following transmission conditions:

$$
\left\{\begin{array}{l}
\llbracket U^{1} \rrbracket=0, \quad \llbracket \mathcal{T}_{2}^{1}-\frac{\partial \mathcal{M}_{21}^{1}}{\partial \bar{x}_{1}} \rrbracket=\mathrm{I}_{\mathrm{d}} \frac{\partial^{4} U^{0}}{\partial \bar{x}_{1}^{4}}-\mathcal{F}_{0}^{0}, \\
\mathrm{D} \llbracket \frac{\partial U^{1}}{\partial \bar{x}_{2}} \rrbracket=-\mathrm{C}_{\mathrm{d}} \mathcal{M}_{22}^{0}, \quad \llbracket \mathcal{M}_{22}^{1} \rrbracket=-6 \mathrm{~J}_{\mathrm{d}} \frac{\partial \mathcal{M}_{21}^{0}}{\partial \bar{x}_{1}},
\end{array}\right.
$$

which differ from the usual ones because of the presence of a boundary layer. That requires to solve the bending boundary layer problem (67) and the twist boundary layer problem (68) to obtain the coefficients $\mathrm{C}_{\mathrm{d}}$ and $\mathrm{J}_{\mathrm{d}}$ whereas the coefficient $\mathrm{I}_{\mathrm{d}}$ is given by $(80)$.

Let us note that the sign of the coefficients $C_{d}, J_{d}$ and $I_{d}$ will depend on the type of defects considered as we will see in the next subsection.

5.7 Study of the boundary layer coefficients $C_{d}, J_{d}$ and $I_{d}$

One first considers the twist boundary layer problem (68) which gives $J_{d}$. Let us show that $J_{d}$ can also read as

$$
\mathrm{J}_{\mathrm{d}}=8 \int_{\mathrm{Y}-\mathrm{Y}_{0}} y_{3}^{2} d \mathbf{y}-2 \int_{\mathrm{Y}} \nabla \mathrm{W}^{\mathrm{d}}(\mathbf{y}) \cdot \nabla \mathrm{W}^{\mathrm{d}}(\mathbf{y}) d \mathbf{y}
$$

Using (68), one successively gets the following relations:

$$
\begin{aligned}
0 & =-\int_{\mathrm{Y}} \Delta \mathrm{W}^{\mathrm{d}}\left(\mathrm{W}^{\mathrm{d}}+y_{2} y_{3}\right) d \mathbf{y}=\int_{\mathrm{Y}} \nabla \mathrm{W}^{\mathrm{d}} \cdot\left(\nabla \mathrm{W}^{\mathrm{d}}+\nabla\left(y_{2} y_{3}\right)\right) d \mathbf{y}-\int_{\partial_{+} \mathrm{Y}} 2 y_{3} n_{2}\left(\mathrm{~W}^{\mathrm{d}}+y_{2} y_{3}\right) d s \\
& =\int_{\mathrm{Y}}\left(\nabla \mathrm{W}^{\mathrm{d}} \cdot \nabla \mathrm{W}^{\mathrm{d}}+y_{2} \frac{\partial \mathrm{W}^{\mathrm{d}}}{\partial y_{3}}+y_{3} \frac{\partial \mathrm{W}^{\mathrm{d}}}{\partial y_{2}}\right) d \mathbf{y}-\int_{\mathrm{Y}} 2 y_{3} \frac{\partial \mathrm{W}^{\mathrm{d}}}{\partial y_{2}} d \mathbf{y}-\int_{\mathrm{Y}-\mathrm{Y}_{0}} 2 y_{3}^{2} d \mathbf{y} \\
& =\int_{\mathrm{Y}}\left(\nabla \mathrm{W}^{\mathrm{d}} \cdot \nabla \mathrm{W}^{\mathrm{d}}+y_{2} \frac{\partial \mathrm{W}^{\mathrm{d}}}{\partial y_{3}}-y_{3} \frac{\partial \mathrm{W}^{\mathrm{d}}}{\partial y_{2}}\right) d \mathbf{y}-\int_{\mathrm{Y}-\mathrm{Y}_{0}} 2 y_{3}^{2} d \mathbf{y} .
\end{aligned}
$$

Inserting this last equality into (75) leads to (81). One immediately deduces from (81) that $J_{\mathrm{d}}$ is negative when the defect is a geometrical weakening because in that case $\int_{\mathrm{Y}-\mathrm{Y}_{0}} y_{3}^{2} d \mathbf{y}=-\int_{\mathrm{Y}_{0} \backslash \mathrm{Y}} y_{3}^{2} d \mathbf{y}<0$. Let us prove on the other hand that $J_{d}$ is positive when the defect is a geometrical stiffening. For that one uses the theorem of the complementary energy. Specifically, let $\mathcal{S}$ be the set of statically admissible vector fields for the twist boundary layer problem, i.e. ,

$$
\mathcal{S}=\left\{\mathbf{T}=\left(T_{2}, T_{3}\right) \in L^{2}(\mathrm{Y})^{2}: \operatorname{div}_{\mathbf{y}} \mathbf{T}=0 \text { in } \mathrm{Y}, \mathbf{T} \cdot \mathbf{n}=2 y_{3} n_{2} \text { on } \partial_{+} \mathrm{Y}\right\},
$$

and let $\mathcal{P}^{*}(\mathbf{T})=\frac{1}{2} \int_{\mathrm{Y}} \mathbf{T} \cdot \mathbf{T} d \mathbf{y}$ be the complementary energy associated with $\mathbf{T} \in \mathcal{S}$. The theorem of the complementary energy says that $\nabla \mathrm{W}^{\mathrm{d}}$ (which belongs to $\mathcal{S}$ ) is the (unique) minimiser of $\mathcal{P}^{*}$ over $\mathcal{S}$. Accordingly, one gets

$$
\int_{\mathrm{Y}} \nabla \mathrm{W}^{\mathrm{d}} \cdot \nabla \mathrm{W}^{\mathrm{d}} d \mathbf{y} \leq \int_{\mathrm{Y}} \mathbf{T} \cdot \mathbf{T} d \mathbf{y}, \quad \forall \mathbf{T} \in \mathcal{S}
$$

Assuming that the defect is a geometrical stiffening and hence that $\mathrm{Y}_{0} \subset \mathrm{Y}$, let us set

$$
\mathbf{T}= \begin{cases}\mathbf{0} & \text { in } \mathrm{Y}_{0}, \\ 2 y_{3} \mathbf{e}_{2} & \text { in } \mathrm{Y} \backslash \mathrm{Y}_{0}\end{cases}
$$

One easily checks that $\mathbf{T} \in \mathcal{S}$ (only $T_{3}$ has to be continuous at $y_{3}=1 / 2$ ) and since $\int_{\mathrm{Y}} \mathbf{T} \cdot \mathbf{T} d \mathbf{y}=\int_{\mathrm{Y} \backslash \mathrm{Y}_{0}} 4 y_{3}^{2} d \mathbf{y}$ one obtains the inequality $\mathrm{J}_{\mathrm{d}} \geq 0$. The equality can hold only when $\mathbf{T}=\nabla \mathrm{W}^{\mathrm{d}}$.

One now considers the bending boundary layer problem (67) which gives $C_{d}$. Let us show that $C_{d}$ can read as

$$
\mathrm{C}_{\mathrm{d}}=24 \int_{\mathrm{Y}-\mathrm{Y}_{0}} y_{3}^{2} d \mathbf{y}-24\left(1-\nu^{2}\right) \int_{\mathrm{Y}} \boldsymbol{\Sigma}^{\mathbf{d}} \cdot \varepsilon_{\mathbf{y}}\left(\mathbf{V}^{\mathbf{d}}\right) d \mathbf{y} .
$$


Introducing the fields $\mathbf{V}^{*}(\mathbf{y})=y_{2} y_{3} \mathbf{e}_{2}-\left(\frac{y_{2}^{2}}{2}+\frac{\nu y_{3}^{2}}{2(1-\nu)}\right) \mathbf{e}_{3}$ and $\boldsymbol{\Sigma}^{*}(\mathbf{y})=\frac{y_{3}}{1-\nu^{2}}\left(\nu \mathbf{e}_{1} \otimes \mathbf{e}_{1}+\mathbf{e}_{2} \otimes \mathbf{e}_{2}\right)$ which are related by $\varepsilon_{\mathbf{y}}\left(\mathbf{V}^{*}\right)=(1+\nu) \boldsymbol{\Sigma}^{*}-\nu \operatorname{Tr} \boldsymbol{\Sigma}^{*} \mathbf{I}$, using (67), the behavior at infinity of $\mathbf{V}^{\mathbf{d}}$ and the definition (70) of $\mathbf{C}_{\mathbf{d}}$ one gets

$$
\begin{aligned}
0 & =-\int_{\mathrm{Y}} \operatorname{div}_{\mathbf{y}} \boldsymbol{\Sigma}^{\mathbf{d}} \cdot\left(\mathbf{V}^{\mathbf{d}}+\mathbf{V}^{*}\right) d \mathbf{y}=\int_{\mathrm{Y}}\left(\boldsymbol{\Sigma}^{\mathbf{d}} \cdot \boldsymbol{\varepsilon}_{\mathbf{y}}\left(\mathbf{V}^{\mathbf{d}}\right)+\boldsymbol{\Sigma}^{*} \cdot \boldsymbol{\varepsilon}_{\mathbf{y}}\left(\mathbf{V}^{\mathbf{d}}\right)\right) d \mathbf{y}-\int_{\partial_{+} \mathrm{Y}} \frac{y_{3} n_{2}}{1-\nu^{2}}\left(V_{2}^{d}+y_{2} y_{3}\right) d s \\
& =\int_{\mathrm{Y}} \boldsymbol{\Sigma}^{\mathbf{d}} \cdot \boldsymbol{\varepsilon}_{\mathbf{y}}\left(\mathbf{V}^{\mathbf{d}}\right) d \mathbf{y}+\int_{\mathrm{Y}} \frac{y_{3}}{1-\nu^{2}} \frac{\partial V_{2}^{d}}{\partial y_{2}} d \mathbf{y}-\int_{\partial_{+} \mathrm{Y}} \frac{y_{3}}{1-\nu^{2}} V_{2}^{d} n_{2} d s-\int_{\mathrm{Y}-Y_{0}} \frac{y_{3}^{2}}{1-\nu^{2}} d \mathbf{y} \\
& =\int_{\mathrm{Y}} \boldsymbol{\Sigma}^{\mathbf{d}} \cdot \varepsilon_{\mathbf{y}}\left(\mathbf{V}^{\mathbf{d}}\right) d \mathbf{y}+\mathrm{C}_{\mathbf{d}} \int_{0}^{1 / 2} \frac{y_{3}^{2}}{1-\nu^{2}} d y_{3}-\int_{\mathrm{Y}-Y_{0}} \frac{y_{3}^{2}}{1-\nu^{2}} d \mathbf{y}
\end{aligned}
$$

and this latter equality gives (82). One immediately deduces from (82) that $C_{d}$ is negative when the defect is a geometrical weakening. Let us prove on the other hand that $C_{d}$ is positive when the defect is a geometrical stiffening by using the theorem of the complementary energy again. The set of statically admissible (in-plane) stress fields for the bending boundary layer problem is defined by

$$
\mathcal{S}=\left\{\boldsymbol{\Sigma}=\left(\begin{array}{ccc}
\Sigma_{11} & 0 & 0 \\
0 & \Sigma_{22} & \Sigma_{23} \\
0 & \Sigma_{23} & \Sigma_{33}
\end{array}\right): \Sigma_{i j} \in L^{2}(\mathrm{Y}), \operatorname{div}_{\mathbf{y}} \boldsymbol{\Sigma}=0 \text { in } \mathrm{Y}, \Sigma_{33}=0 \text { at } y_{3}=0, \boldsymbol{\Sigma} \mathbf{n}=\frac{y_{3} n_{2}}{1-\nu^{2}} \text { on } \partial_{+} \mathrm{Y}\right\}
$$

and the complementary energy associated with $\boldsymbol{\Sigma} \in \mathcal{S}$ is given by

$$
\mathcal{P}^{*}(\boldsymbol{\Sigma})=\frac{1}{2} \int_{\mathrm{Y}}\left((1+\nu) \boldsymbol{\Sigma} \cdot \boldsymbol{\Sigma}-\nu(\operatorname{Tr} \boldsymbol{\Sigma})^{2}\right) d \mathbf{y}
$$

The theorem of the complementary energy says that $\boldsymbol{\Sigma}^{\mathbf{d}}$ is the (unique) minimiser of $\mathcal{P}^{*}$ over $\mathcal{S}$. Accordingly, one gets $\int_{\mathbf{Y}} \boldsymbol{\Sigma}^{\mathbf{d}} \cdot \varepsilon_{\mathbf{y}}\left(\mathbf{V}^{\mathbf{d}}\right) d \mathbf{y}=2 \mathcal{P}^{*}\left(\boldsymbol{\Sigma}^{\mathbf{d}}\right) \leq 2 \mathcal{P}^{*}(\boldsymbol{\Sigma})$ for every $\boldsymbol{\Sigma} \in \mathcal{S}$. Assuming that the defect is a geometrical stiffening, let us consider the following statically admissible stress field $\boldsymbol{\Sigma}$ :

$$
\boldsymbol{\Sigma}= \begin{cases}\mathbf{0} & \text { in } \mathrm{Y}_{0} \\ \frac{y_{3}}{1-\nu^{2}}\left(\nu \mathbf{e}_{1} \otimes \mathbf{e}_{1}+\mathbf{e}_{2} \otimes \mathbf{e}_{2}\right) & \text { in } \mathrm{Y} \backslash \mathrm{Y}_{0}\end{cases}
$$

Since $2 \mathcal{P}^{*}(\boldsymbol{\Sigma})=\int_{\mathrm{Y} \backslash \mathrm{Y}_{0}} \frac{y_{3}^{2}}{1-\nu^{2}} d \mathbf{y}$ one obtains the inequality $\mathrm{C}_{\mathrm{d}} \geq 0$ and the equality can hold only when $\boldsymbol{\Sigma}^{\mathbf{d}}=\boldsymbol{\Sigma}$.

By (80) the sign of $I_{d}$ is obvious: $I_{d}$ is positive in the case of a geometrical stiffening, and negative in the case of a weakening. Therefore, one can summarise that study of the coefficients by the following proposition.

Proposition 4 The three coefficients $\mathrm{C}_{\mathrm{d}}, \mathrm{J}_{\mathrm{d}}$ and $\mathrm{I}_{\mathrm{d}}$ involved in the transmission conditions are positive when the defect is a geometrical stiffening whereas they are negative in the case of a geometrical weakening.

To illustrate the dependence of the geometry of the defect on the three coefficients, we consider a defect whose width is equal to the thickness of the plate and whose height is left as a parameter. Specifically, the defect is the rectangle $(-1 / 2,1 / 2) \times(1 / 2,1 / 2+\bar{a})$ with $\bar{a}>0$ in the case of a stiffening, or the rectangle $(-1 / 2,1 / 2) \times(1 / 2+\overline{\mathrm{a}}, 1 / 2)$ with $-1 / 2<\overline{\mathrm{a}}<0$ in the case of a weakening. The three coefficients are computed numerically (by a finite element code for $C_{d}$ and $\left.J_{d}\right)$ as a function of $\bar{a}$, the Poisson ratio being equal to $0.25\left(C_{d}\right.$ and $I_{d}$ depend on $\nu$ but not $\left.J_{d}\right)$. Their graph is plotted on Figure 6. Their sign is conform to Proposition 4. When the height $\bar{a}$ of the stiffener grows, $C_{d}$ tends to a limit whereas the growth of $J_{d}$ and $I_{d}$ are respectively linear and quadratic. On the other hand, in the case of a weakening, when the height of the defect tends to the thickness of the plate, then $C_{d}$ tends to $-\infty$ whereas $I_{d}$ and $J_{d}$ tend to a finite limit. 

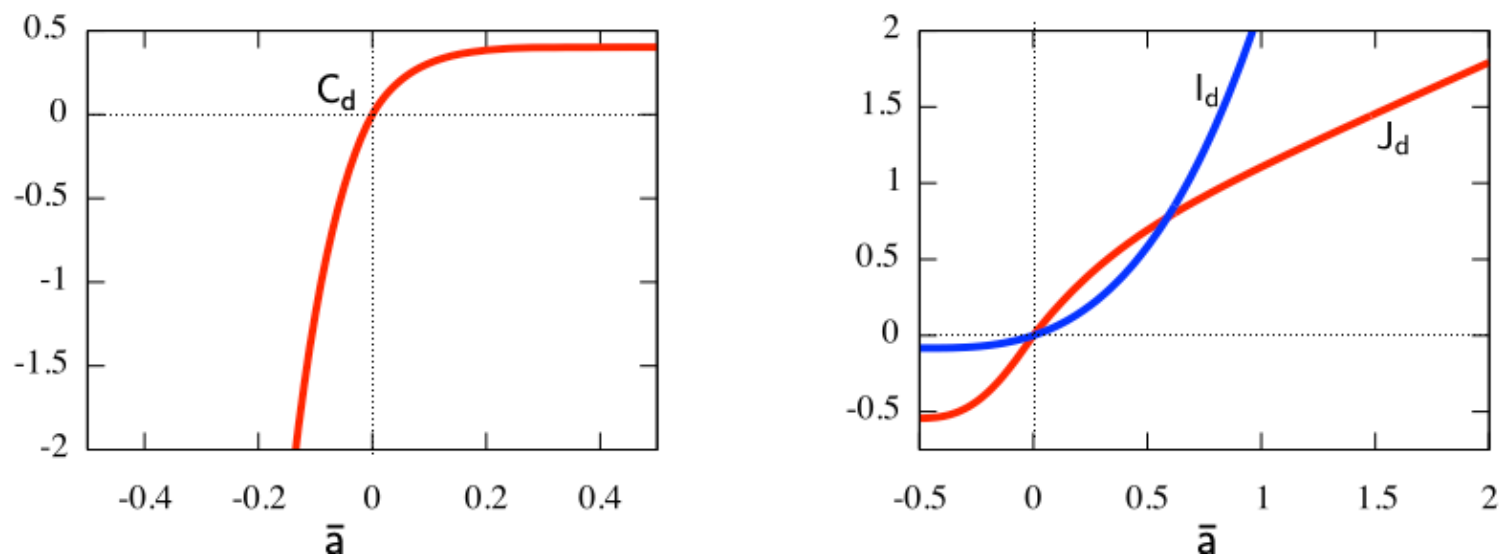

Fig. 6 Graphs of the coefficients $C_{d}, I_{d}$ and $J_{d}$ as a function of the height $\bar{a}$ of the defect when $\nu=0.25$, $\bar{a}>0$ corresponds to a stiffening, $\bar{a}<0$ to a weakening. While $I_{d}$ is a geometric moment, the coefficients $C_{d}$ and $J_{d}$ can be interpreted as effective rotational and torsional stiffnesses, respectively.

\section{The model of a bent elastic plate including boundary layer effects}

Throughout this section, the double brackets denote the jump whereas the angles denote the mean value for discontinuous quantities across the line of defect, i.e. ,

$$
\llbracket \varphi \rrbracket=\varphi^{+}-\varphi^{-}, \quad\langle\varphi\rangle=\frac{1}{2}\left(\varphi^{+}+\varphi^{-}\right) .
$$

6.1 The first boundary value problem

We have shown that the leading terms of the expansion, i.e., $U^{0}$ for the displacements, $\boldsymbol{T}^{0}$ and $\mathcal{M}^{0}$ for the shear forces and the bending moments are solutions of the following boundary problem:

\begin{tabular}{|c|c|c|c|}
\hline $\operatorname{in} \Omega_{0} \backslash I$ & on $\partial_{c} \Omega_{0}$ & on $\partial_{f} \Omega_{0}$ & \multicolumn{1}{c|}{ on $I$} \\
\hline$\left\{\begin{array}{l}\operatorname{div} \mathcal{T}^{0}+\mathcal{F}^{0}=0, \\
\operatorname{div} \mathcal{M}^{0}+\mathcal{T}^{0}=0, \\
\mathcal{M}_{\alpha \beta}^{0}=\mathrm{D}\left(\nu \Delta U^{0} \delta_{\alpha \beta}+(1-\nu) U_{, \alpha \beta}^{0}\right),\end{array}\right.$ & $\left\{\begin{array}{l}U^{0}=0, \\
U_{, 1}^{0}=0,\end{array}\right.$ & $\left\{\begin{array}{l}\mathcal{T}_{2}^{0}-\mathcal{M}_{21,1}^{0}=0, \\
\mathcal{M}_{22}^{0}=0,\end{array}\right.$ & $\left\{\begin{array}{l}\llbracket U^{0} \rrbracket=\llbracket \mathcal{T}_{2}^{0}-\mathcal{M}_{21,1}^{0} \rrbracket=0, \\
\llbracket U_{, 2}^{0} \rrbracket=\llbracket \mathcal{M}_{22}^{0} \rrbracket=0,\end{array}\right.$ \\
\hline
\end{tabular}

where $I=(-1 / 2,+1 / 2) \times\{0\}$ denotes the line of defect, $\partial_{c} \Omega_{0}=\{ \pm 1 / 2\} \times(-\bar{\ell} / 2,+\bar{\ell} / 2)$ the clamped part of the boundary and $\partial_{f} \Omega_{0}=(-1 / 2,+1 / 2) \times\{ \pm \bar{\ell} / 2\}$ the free part of the boundary. Therefore, at first order, one recovers the usual model of Love-Kirchhoff which does not account for the presence of the defect nor the boundary layer effects at the boundary. By standard arguments [10], one can show that the displacement field $U^{0}$ is the minimizer of the potential energy $\mathcal{P}^{0}$ over the set $\mathcal{V}^{0}$ of kinematically displacement fields with $\mathcal{P}^{0}$ and $\mathcal{V}^{0}$ defined by

$$
\begin{gathered}
\mathcal{P}^{0}(V)=\int_{\Omega_{0}} \frac{\mathrm{D}}{2}\left(\nu(\Delta V)^{2}+(1-\nu) \nabla^{2} V \cdot \nabla^{2} V\right) d \overline{\mathbf{x}}-\int_{\Omega_{0}} \mathcal{F}^{0} V d \overline{\mathbf{x}}, \\
\mathcal{V}^{0}=\left\{V \in H^{2}\left(\Omega_{0}\right), V=\partial V / \partial n=0 \text { on } \partial_{c} \Omega_{0}\right\} .
\end{gathered}
$$

The Sobolev space $H^{2}\left(\Omega_{0}\right)$ is the natural space of functions of finite energy where the solution has to be searched [10]. The existence and the uniqueness of $U^{0}$ is guaranteed as soon as the loading $\mathcal{F}^{0}$ is smooth enough, for instance $\mathcal{F}^{0}$ in $L^{2}\left(\Omega_{0}\right)$ is sufficient. The functions of $H^{2}\left(\Omega_{0}\right)$ have a trace on the boundary which belongs (at least) to $H^{3 / 2}\left(\partial \Omega_{0}\right)$ and their normal derivative $\partial V / \partial n$ belongs (at least) to $H^{1 / 2}\left(\partial \Omega_{0}\right)$. Accordingly, the clamped conditions on the sides $\bar{x}_{1}= \pm 1 / 2$ must be understood in that sense of traces. The components of the bending moment tensor field $\mathcal{M}^{0}$ are (at least) in $L^{2}\left(\Omega_{0}\right)$. When $\mathcal{F}^{0}$ is a smooth (infinitely differentiable) function of $\overline{\mathbf{x}}$, so is $U^{0}$ and hence all the mechanical fields are infinitely differentiable in the open set $\Omega_{0}$. That regularity remains true on the boundary except at the four corners $( \pm 1 / 2, \pm \bar{\ell} / 2)$ where weak singularities can occur because of the change of the type of boundary conditions. Specifically, the bending moments and the shear forces could tend to infinity when $\overline{\mathbf{x}}$ tends to one corner. As we will see in the next subsection, the discussion to know whether such a weak singularity exists is important when one considers the problem for $U^{1}$. 
6.2 The second boundary value problem

We assume from now that the loading $\mathcal{F}^{0}$ is a smooth function of $\bar{x}$ and hence that the first boundary value problem admits a smooth solution in the closure of $\Omega_{0}$ except maybe at the four corners. Therefore, assuming that $U^{0}, \mathcal{T}^{0}$ and $\mathcal{M}^{0}$ are known (both inside the domain $\Omega_{0}$ and on its boundary), the fields $U^{1}, \mathcal{T}^{1}$ and $\mathcal{M}^{1}$ have to satisfy the following boundary value problem:

$$
\text { in } \Omega_{0} \backslash I:\left\{\begin{array}{l}
\operatorname{div} \mathcal{T}^{1}=0, \quad \operatorname{div} \mathcal{M}^{1}+\mathcal{T}^{1}=0 \\
\mathcal{M}_{\alpha \beta}^{1}=\mathrm{D}\left(\nu \Delta U^{1} \delta_{\alpha \beta}+(1-\nu) U_{, \alpha \beta}^{1}\right)
\end{array}\right.
$$

\begin{tabular}{c}
\hline on $\partial_{c} \Omega_{0}$ \\
\hline $\begin{array}{c}\text { on } \partial_{f} \Omega_{0} \\
\text { on } I\end{array}$ \\
$\left\{\begin{array}{l}U^{1}=0 \\
\mathrm{D} U_{, n}^{1}=\mathrm{C}_{\mathrm{c}} \mathcal{M}_{n n}^{0}\end{array} \quad\left\{\begin{array}{l}\llbracket \mathcal{T}_{n}^{1}-\mathcal{M}_{n t, t}^{1}=0 \\
\mathcal{M}_{n n}^{1}=-6 \mathrm{~J}_{\mathrm{f}} \mathcal{M}_{n t, t}^{0}\end{array} \quad\left\{\begin{array}{l}\llbracket \mathcal{T}_{2}^{1}-\mathcal{M}_{21,1}^{1} \rrbracket=\mathrm{I}_{\mathrm{d}} U_{, 1111}^{0}-\mathcal{F}_{0}^{0} \\
\mathrm{D} \llbracket U_{, 2}^{1} \rrbracket=-\mathrm{C}_{\mathrm{d}} \mathcal{M}_{22}^{0} \\
\llbracket \mathcal{M}_{22}^{1} \rrbracket=-6 \mathrm{~J}_{\mathrm{d}} \mathcal{M}_{21,1}^{0}\end{array}\right.\right.\right.$ \\
\hline
\end{tabular}

where the indices $n$ and $t$ refer to the outer normal and tangent vectors at the considered boundary. Let us discuss the existence of a solution for $U^{1}$ in the natural space $H^{2}\left(\Omega_{0} \backslash I\right)$. (Since the normal derivative of $U^{1}$ is discontinuous on $I, U^{1}$ cannot belong to $H^{2}\left(\Omega_{0}\right)$.) The kinematic data and the loading of the plate are smooth on each side of the plate and on the defect line by virtue of the assumption on $\mathcal{F}^{0}$. But it remains to check whether $U^{0}$ has a weak singularity at the four corners of the plate and whether a misfit between the data occurs at the two end points $\mathbf{D}^{ \pm}=( \pm 1 / 2,0)$ of the line of defect.

Let us first study the question of the singularity of $U^{0}$ at a corner. Specifically, we consider the corner $(-1 / 2,-\bar{\ell} / 2)$ and introduce the polar coordinates $(r, \theta)$ defined by

$$
\bar{x}_{1}+1 / 2=r \sin \theta, \quad \bar{x}_{2}+\bar{\ell} / 2=r \cos \theta, \quad r>0, \quad \theta \in[0, \pi / 2],
$$

so that $\theta=0$ corresponds to the clamped side and $\theta=\pi / 2$ to the free side. The singular part of $U^{0}$, denoted $U_{S}^{0}$, is searched of the form $U_{S}^{0}(\overline{\mathbf{x}})=r^{p} \mathbf{u}(\theta)$ with $1<p<2$ (the complete analysis of the singularities is outside the scope of this paper and we suppose that the singularities have necessarily that form, see $[13,22]$ for a more comprehensive analysis) so that $U_{S}^{0}$ is in $H^{2}\left(\Omega_{0}\right)$ but not in $H^{3}\left(\Omega_{0}\right)$. When such a singularity exists, bending moments associated with the singular part are of the form $r^{p-2} \mathrm{~m}_{\alpha \beta}(\theta)$ and hence are infinite at the corner. Consequently, the boundary conditions for $U^{1}$ on the clamped side will read

$$
U^{1}(r, 0)=0, \quad \frac{\partial U^{1}}{\partial \theta}(r, 0)=\frac{\mathrm{C}_{\mathrm{c}}}{\mathrm{D}} \mathrm{m}_{\theta \theta}(0) r^{p-1}+\cdots,
$$

and cannot be satisfied by a function of $H^{2}\left(\Omega_{0} \backslash I\right)$ when $p<2$ and $\mathrm{C}_{\mathrm{c}} \mathrm{m}_{\theta \theta}(0) \neq 0$. In the same manner, the boundary conditions for $\mathcal{M}^{1}$ and $\mathcal{T}^{1}$ on the free side read

$$
\mathcal{T}_{\theta}^{1}(r, \pi / 2)-\frac{\partial \mathcal{M}_{\theta r}^{1}}{\partial r}(r, \pi / 2)=0, \quad \mathcal{M}_{\theta \theta}^{1}(r, \pi / 2)=6(2-p) \mathrm{J}_{\mathrm{f}} \mathrm{m}_{\theta r}(\pi / 2) r^{p-3}+\cdots
$$

and cannot be satisfied by a function $U^{1}$ in $H^{2}\left(\Omega_{0} \backslash I\right)$ when $p<2$ and $\mathrm{m}_{\theta r}(\pi / 2) \neq 0$. On the other hand, those boundary conditions are compatible with $U^{1}$ in $H^{2}\left(\Omega_{0} \backslash I\right)$ when $p>2$ whereas the case $p=2$ is a particular case that one must study separately. So let us search in what condition a singularity exists with $p<2$. From the equilibrium condition $\Delta^{2} U_{S}^{0}=0$ and the kinematic conditions $\mathrm{u}(0)=\mathrm{u}^{\prime}(0)=0$ one gets that the angular function must read

$$
\mathrm{u}(\theta)=\mathrm{a}(\cos (p-2) \theta-\cos p \theta)+\mathrm{b}(p \sin (p-2) \theta-(p-2) \sin p \theta)
$$

where $\mathrm{a}$ and $\mathrm{b}$ are two arbitrary constants. The static conditions at $\theta=\pi / 2$ give the equation for $p$ and a relation between $a$ and $b$ :

$$
\left\{\begin{array}{l}
2(1-\nu)^{2} p(2-p)+3+6 \nu-\nu^{2}=(1-\nu)(3+\nu) \cos p \pi \\
(2-(p-1) \nu) \mathrm{a}+(3-\nu-p(1-\nu)) \tan (p \pi / 2) \mathrm{b}=0
\end{array}\right.
$$

The solution for the exponent $p$ is plotted in Figure 7 as function of $\nu$. One sees that $p<2$ only for negative Poisson's ratios. When $\nu=0$ one directly shows that $p=2$ is solution but the associated field reads $U_{s}^{0}(\overline{\mathbf{x}})=\mathrm{a}\left(\bar{x}_{1}+1 / 2\right)^{2}$ and hence is not singular. So one can conclude that if the Poisson ratio is non negative, then boundary conditions at the four corners can be satisfied by a function $U^{1}$ in $H^{2}\left(\Omega_{0} \backslash I\right)$. 

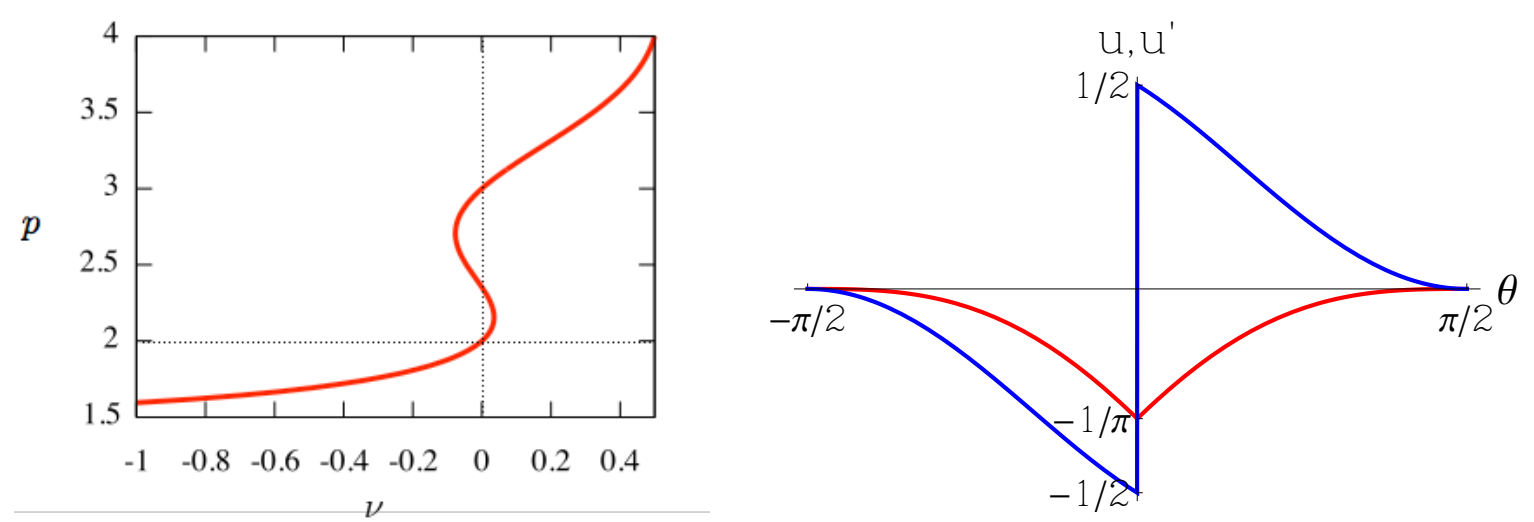

Fig. 7 Left:Value of the exponent $p$ of a singularity of the type $r^{p} u(\theta)$ for $U^{0}$ at a corner of the plate in function of the Poisson ratio $\nu$. When $p<2$, then $U^{0}$ is not regular enough in order that $U^{1}$ be in $H^{2}\left(\Omega_{0}\right)$. It is the case when $-1<\nu<0$.

Right: angular function $\mathbf{u}\left(\right.$ red) and its derivative $\mathbf{u}^{\prime}$ (blue) entering in the singular part of $U^{1}$ at the end points $\mathbf{D} \pm$ of the line of defect.

Let us now study the question of the boundary conditions near the end points $\mathbf{D}^{ \pm}$. The fixation condition on the clamped side gives $U^{1}=0$ and hence $U_{, 2}^{1}=0$ on $\bar{x}_{1}= \pm 1 / 2$. On the other hand the jump condition for the rotation gives $\mathrm{D} \llbracket U_{, 2}^{1} \rrbracket=-\mathrm{C}_{\mathrm{d}} \mathcal{M}_{22}^{0}$ on $\bar{x}_{2}=0$. Therefore if $\mathcal{M}_{22}^{0}\left(\mathbf{D}^{ \pm}\right) \neq 0$, there exists a misfit of the data at $\mathbf{D}^{ \pm}$and $\llbracket U_{, 2}^{1} \rrbracket$ is not defined there. Such boundary conditions cannot be satisfied by a function in $H^{2}\left(\Omega_{0} \backslash I\right)$ but only by a less regular field. Let us construct such a singular field. Specifically, we consider the end point $\mathbf{D}^{-}=(-1 / 2,0)$ and introduce the polar coordinates $(r, \theta)$ defined by

$$
\bar{x}_{1}+1 / 2=r \cos \theta, \quad \bar{x}_{2}=r \sin \theta, \quad r>0, \quad \theta \in[-\pi / 2, \pi / 2],
$$

so that $\theta=0$ corresponds to the line of defect and $\theta= \pm \pi / 2$ to the clamped side. Since $U^{0}$ is smooth in the neighbourhood of $\mathbf{D}^{-}$, the boundary and jump conditions near $\mathbf{D}^{-}$(i.e. , for small $r$ ) can read as:

\begin{tabular}{cc} 
at $\theta= \pm \pi / 2$ & at $\theta=0$ \\
\hline$U^{1}=0$, & at $\theta=0$ \\
$\mathrm{D} U_{, \theta}^{1}= \pm \mathrm{C}_{\mathrm{c}} \mathcal{M}_{11}^{0}\left(\mathbf{D}^{-}\right) r+\cdots, \mathrm{D} \llbracket U_{, \theta}^{1} \rrbracket=-\mathrm{C}_{\mathrm{d}} \mathcal{M}_{22}^{0}\left(\mathbf{D}^{-}\right) r+\cdots$, & $\llbracket \mathcal{T}_{\theta}^{1}-\mathcal{M}_{\theta r, r}^{1} \rrbracket=\mathrm{I}_{\mathrm{d}} U_{, 1111}^{0}\left(\mathbf{D}^{-}\right)-\mathcal{F}_{0}^{0}(-1 / 2)+\cdots$, \\
\hline
\end{tabular}

where the dots stand for higher order terms in $r$. Accordingly, let us search the singular part of $U^{1}$ of the form $U^{S}(r, \theta)=r \mathrm{u}(\theta)$ in such a manner that, in the neighbourhood of $\mathbf{D}^{-}, U^{1}$ can read as

$$
U^{1}(\overline{\mathbf{x}})=-\frac{\mathrm{C}_{\mathrm{d}}}{\mathrm{D}} \mathcal{M}_{22}^{0}\left(\mathbf{D}^{-}\right) r \mathbf{u}(\theta)-\frac{\mathrm{C}_{\mathrm{c}}}{\mathrm{D}} \mathcal{M}_{11}^{0}\left(\mathbf{D}^{-}\right)\left(\bar{x}_{1}+1 / 2\right)+\cdots
$$

The singular part $\mathcal{M}^{S}$ of the bending moments are of the order of $r^{-1}$ and the singular part $\mathcal{T}^{S}$ of the shear

\begin{tabular}{|c|c|c|}
\hline for $\theta \in(-\pi / 2, \pi / 2) \backslash\{0\}$ & at $\theta= \pm \pi / 2$ & at $\theta=0$ \\
\hline$\Delta^{2} U^{S}=0$ & $\left\{\begin{array}{l}U^{S}=0 \\
U_{, \theta}^{S}=0\end{array}\right.$ & $\left\{\begin{array}{l}\llbracket \mathcal{T}_{\theta}^{S}-\mathcal{M}_{\theta r, r}^{S} \rrbracket=0 \\
\llbracket \mathcal{M}_{\theta \theta}^{S} \rrbracket=0\end{array}\right.$ \\
\hline
\end{tabular}
forces are of the order of $r^{-2}$. Therefore the singular part of $U^{1}$ must satisfy

After some calculations which are not reproduced here the singular part is determined in a unique way, see the graphs of $\mathbf{u}$ and $d \mathbf{u} / d \theta$ on Figure 7 , and eventually reads

$$
U^{S}(r, \theta)=\frac{r}{\pi}\left(\left(\frac{\pi}{2}-|\theta|\right)|\sin \theta|-\cos \theta\right)
$$

So $U^{S}$ belongs to $H^{1}(\Omega)$ but not to $H^{2}(\Omega \backslash I)$, as expected. The associated bending moments and shear forces are infinite at $\mathbf{D}^{-}$and given by

$$
\mathcal{M}^{S}(r, \theta)=-\frac{2 \mathrm{D} \cos \theta}{\pi r}\left(\nu \mathbf{e}_{r} \otimes \mathbf{e}_{r}+\mathbf{e}_{\theta} \otimes \mathbf{e}_{\theta}\right), \quad \mathcal{T}^{S}(r, \theta)=-\frac{2 \mathrm{D}}{\pi r^{2}}\left(\cos \theta \mathbf{e}_{r}+\sin \theta \mathbf{e}_{\theta}\right) .
$$


One can proceed in the same manner at the end point $\mathbf{D}^{+}$. Introducing the polar coordinates $(r, \theta): 1 / 2-\bar{x}_{1}=r \cos \theta$, $\bar{x}_{2}=-r \sin \theta$ with $r>0$ and $\theta \in[-\pi / 2, \pi / 2]$, one gets that, in the neighbourhood of $\mathbf{D}^{+}, U^{1}$ can read as

$$
U^{1}(\overline{\mathbf{x}})=-\frac{\mathrm{C}_{\mathrm{d}}}{\mathrm{D}} \mathcal{M}_{22}^{0}\left(\mathbf{D}^{+}\right) U^{S}(r, \theta)-\frac{\mathrm{C}_{\mathrm{c}}}{\mathrm{D}} \mathcal{M}_{11}^{0}\left(\mathbf{D}^{+}\right)\left(1 / 2-\bar{x}_{1}\right)+\cdots
$$

where $U^{S}$ is still given by $(85)$.

Finally, reintroducing the Cartesian coordinates, the singular part of $U^{1}$ is the combination of the singularities found at the two end points and reads

$$
\begin{aligned}
U^{1 S}(\overline{\mathbf{x}}) & =\frac{\mathrm{C}_{\mathrm{d}}}{\pi \mathrm{D}} \mathcal{M}_{22}^{0}\left(\mathbf{D}^{-}\right)\left(\bar{x}_{1}+\frac{1}{2}-\left(\frac{\pi}{2}-\arctan \frac{2\left|\bar{x}_{2}\right|}{2 \bar{x}_{1}+1}\right)\left|\bar{x}_{2}\right|\right) \\
& +\frac{\mathrm{C}_{\mathrm{d}}}{\pi \mathrm{D}} \mathcal{M}_{22}^{0}\left(\mathbf{D}^{+}\right)\left(\frac{1}{2}-\bar{x}_{1}-\left(\frac{\pi}{2}-\arctan \frac{2\left|\bar{x}_{2}\right|}{1-2 \bar{x}_{1}}\right)\left|\bar{x}_{2}\right|\right),
\end{aligned}
$$

from which one deduces the singular parts $\mathcal{M}^{1 S}$ and $\mathcal{T}^{1 S}$ of the bending moments and the shear forces. Note that $U^{1 S}, \mathcal{M}^{1 S}$ and $\mathcal{T}^{1 S}$ have a trace on the whole boundary of $\Omega_{0}$ and on the defect. The boundary conditions satisfied by the regular parts must be written accordingly. Specifically, let us decompose $U^{1}, \mathcal{M}^{1}$ and $\mathcal{T}^{1}$ into their regular and singular parts: $U^{1}=U^{1 S}+U^{1 R}, \mathcal{M}^{1}=\mathcal{M}^{1 S}+\mathcal{M}^{1 R}, \mathcal{T}^{1}=\mathcal{T}^{1 S}+\mathcal{T}^{1 R}$. The regular part $U^{1 R}$ has to satisfy the following kinematic conditions on the clamped sides and on the defect:

$$
\begin{array}{cc}
\hline \text { at } \bar{x}_{1}= \pm 1 / 2 & \text { at } \bar{x}_{2}=0 \\
\hline U^{1 R}=-\frac{\mathrm{C}_{\mathrm{d}}}{\pi \mathrm{D}} \mathcal{M}_{22}^{0}\left(\mathbf{D}^{\mp}\right)\left(1-\frac{\pi}{2}\left|\bar{x}_{2}\right|+\bar{x}_{2} \arctan \bar{x}_{2}\right), & \llbracket U^{1 R} \rrbracket=0, \\
\frac{\partial U^{1 R}}{\partial \bar{x}_{1}}=\mp \frac{\mathrm{C}_{\mathrm{d}}}{\pi \mathrm{D}} \frac{\mathcal{M}_{22}^{0}\left(\mathbf{D}^{\mp}\right)}{1+\bar{x}_{2}^{2}} \pm \frac{\mathrm{C}_{\mathrm{c}}}{\mathrm{D}} \mathcal{M}_{11}^{0}, & \llbracket \frac{\partial U^{1 R}}{\partial \bar{x}_{2}} \rrbracket=\frac{\mathrm{C}_{\mathrm{d}}}{\mathrm{D}}\left(\mathcal{M}_{22}^{0}\left(\mathbf{D}^{-}\right)+\mathcal{M}_{22}^{0}\left(\mathbf{D}^{+}\right)-\mathcal{M}_{22}^{0}\right), \\
\hline
\end{array}
$$

\begin{tabular}{|c|c|c|}
\hline in $\Omega_{0} \backslash I$ & at $\bar{x}_{2}= \pm \bar{\ell} / 2$ & at $\bar{x}_{2}=0$ \\
\hline $\begin{array}{l}\operatorname{div} \mathcal{T}^{1 R}=0 \\
\operatorname{div} \mathcal{M}^{1 R}+\mathcal{T}^{1 R}=0\end{array}$ & $\left\{\begin{array}{l}\mathcal{T}_{n}^{1 R}-\mathcal{M}_{n t, t}^{1 R}=\mathcal{M}_{n t, t}^{1 S}-\mathcal{T}_{n}^{1 S} \\
\mathcal{M}_{n n}^{1 R}=-6 \mathrm{~J}_{\mathrm{f}} \mathcal{M}_{n t, t}^{0}-\mathcal{M}_{n n}^{1 S}\end{array}\right.$ & $\left\{\begin{array}{l}\llbracket \mathcal{T}_{2}^{1 R}-\mathcal{M}_{21,1}^{1 R} \rrbracket=\mathrm{I}_{\mathrm{d}} U_{, 1111}^{0}-\mathcal{F}_{0}^{0}, \\
\llbracket \mathcal{M}_{22}^{1 R} \rrbracket=-6 \mathrm{~J}_{\mathrm{d}} \mathcal{M}_{21,1}^{0} .\end{array}\right.$ \\
\hline
\end{tabular}

which are compatible with $U^{1 R} \in H^{2}\left(\Omega_{0} \backslash I\right)$. The regular parts $\mathcal{M}^{1 R}$ and $\mathcal{T}^{1 R}$ have to satisfy the following set of equilibrium and boundary conditions:

Note that the singular parts $\mathcal{M}^{1 S}$ and $\mathcal{T}^{1 S}$ are involved on the free sides where they are smooth functions of $\bar{x}_{1}$. We are in position to state the following existence and uniqueness result for $U^{1}$

Proposition 5 When the Poisson ratio is non negative and when the loading $\mathcal{F}^{0}$ is smooth, the problem (84) admits a unique solution such that the singular part $U^{1 S}$ of $U^{1}$ is given by (86) from which one deduces the singular parts $\mathcal{M}^{1 S}$ and $\mathcal{T}^{1 S}$ of $\mathcal{M}^{1}$ and $\mathcal{T}^{1}$ by

$$
\mathcal{M}^{1 S}=\mathrm{D}\left(\nu \Delta U^{1 S} \mathbf{I}+(1-\nu) \nabla^{2} U^{1 S}\right), \quad \mathcal{T}^{1 S}=-\operatorname{div} \mathcal{M}^{1 S}
$$

The regular part $U^{1 R}$ of $U^{1}$ is the unique minimizer of $\mathcal{P}^{1}$ over the affine subspace $\mathcal{V}^{1}=U^{R}+\mathcal{V}^{0}$ of $H^{2}\left(\Omega_{0} \backslash I\right)$ where

$$
\begin{gathered}
\mathcal{P}^{1}(V)=\int_{\Omega_{0} \backslash I} \frac{\mathrm{D}}{2}\left(\nu(\Delta V)^{2}+(1-\nu) \nabla^{2} V \cdot \nabla^{2} V\right) d \overline{\mathbf{x}}-\mathrm{f}^{1}(V) \\
\mathrm{f}^{1}(V)=\int_{I}\left(6 \mathrm{~J}_{\mathrm{d}} \mathcal{M}_{21,1}^{0}\left\langle V_{, 2}\right\rangle+\left(\mathcal{F}_{0}^{0}-\mathrm{I}_{\mathrm{d}} U_{, 1111}^{0}\right) V\right) d s-\int_{\partial_{f} \Omega_{0}}\left(\left(6 \mathrm{~J}_{\mathrm{f}} \mathcal{M}_{n t, t}^{0}+\mathcal{M}_{n n}^{1 S}\right) V_{, n}+\left(\mathcal{T}_{n}^{1 S}-\mathcal{M}_{n t, t}^{1 S}\right) V\right) d s
\end{gathered}
$$

and $U^{R}$ is a arbitrarily chosen field in $H^{2}\left(\Omega_{0} \backslash I\right)$ which satisfies the kinematic conditions (87). Then the regular parts $\mathcal{M}^{1 R}$ and $\mathcal{T}^{1 R}$ are given by $\mathcal{M}^{1 R}=\mathrm{D}\left(\nu \Delta U^{1 R} \mathbf{I}+(1-\nu) \nabla^{2} U^{1 R}\right)$ and $\mathcal{T}^{1 R}=-\operatorname{div} \mathcal{M}^{1 R}$.

Proof To prove the proposition, let us show first that the following variational equality holds:

$$
\int_{\Omega_{0} \backslash I} \mathcal{M}_{\alpha \beta}^{1 R} V_{, \alpha \beta} d \overline{\mathbf{x}}=\mathrm{f}^{1}(V), \quad \forall V \in \mathcal{V}^{0},
$$


with $\mathcal{M}^{1 R}$ related to $U^{1 R}$ by the constitutive relations. One starts from the equilibrium equation $\mathcal{M}_{\alpha \beta, \alpha \beta}^{1 R}=0$ that is multiplied by $V \in \mathcal{V}^{0}$ (hence $\llbracket V \rrbracket=\llbracket V, 2 \rrbracket=0$ on $I$ ) and integrated over $\Omega_{0} \backslash I$. After two integrations by parts and using the boundary conditions $V=V, n=0$ on $\bar{x}_{1}= \pm 1 / 2$, one gets

$$
\int_{\Omega_{0} \backslash I} \mathcal{M}_{\alpha \beta}^{1 R} V_{, \alpha \beta} d \overline{\mathbf{x}}=\int_{\partial_{f} \Omega_{0}}\left(\mathcal{M}_{n \alpha}^{1 R} V_{, \alpha}+\mathcal{T}_{n}^{1 R} V\right) d s-\int_{I}\left(\llbracket \mathcal{M}_{2 \alpha}^{1 R} \rrbracket V, \alpha+\llbracket \mathcal{T}_{2}^{1 R} \rrbracket V\right) d s .
$$

Decomposing the first term of each integral in the right hand side above into its tangential and normal parts, then integrating by parts the tangential part gives

$$
\int_{\Omega_{0} \backslash I} \mathcal{M}_{\alpha \beta}^{1 R} V_{, \alpha \beta} d \overline{\mathbf{x}}=\int_{\partial_{f} \Omega_{0}}\left(\mathcal{M}_{n n}^{1 R} V_{, n}+\left(\mathcal{T}_{n}^{1 R}-\mathcal{M}_{n t, t}^{1 R}\right) V\right) d s-\int_{I}\left(\llbracket \mathcal{M}_{22}^{1 R} \rrbracket V_{, 2}+\llbracket \mathcal{T}_{2}^{1 R}-\mathcal{M}_{21,1}^{1 R} \rrbracket V\right) d s .
$$

Finally, it suffices to use the boundary conditions (88) satisfied by $\mathcal{M}^{1 R}$ and $\mathcal{T}^{1 R}$ to obtain (89). The existence and uniqueness for $U^{1 R}$ follows by standard arguments, $\mathcal{P}^{1}$ being continuous as well as coercive and $\mathcal{V}^{1}$ being closed in $H^{2}\left(\Omega_{0} \backslash I\right)$.

Therefore $U^{1}$ is not of finite energy because of its singular part. However the determination of $U^{1}$ (including its singular part) is necessary to obtain a good approximation of the real potential energy of the three-dimensional plate up to the order $\eta$. Indeed let us show that the real potential energy $\mathcal{P}^{\eta}$ of the three-dimensional plate with the defect admits the following expansion:

$$
\mathcal{P}^{\eta}=-\frac{1}{2} h L^{2} E\left(\int_{\Omega_{0}} \mathcal{F}^{0}\left(U^{0}+\eta U^{1}\right) d \overline{\mathbf{x}}+\eta \int_{I} \mathcal{F}_{0}^{0} U^{0} d \bar{x}_{1}\right)+o(\eta) \quad \text { with } \quad \lim _{\eta \rightarrow 0} o(\eta) / \eta=0 .
$$

Note that the integral $\int_{\Omega_{0}} \mathcal{F}^{0}(\overline{\mathbf{x}}) U^{1 S}(\overline{\mathbf{x}}) d \overline{\mathbf{x}}$ is finite even though the elastic energy associated to $U^{1 S}$ is infinite. By virtue of the theorem of the potential energy and of the Clapeyron formula, the potential energy of the plate at equilibrium is minus one half of the work done by the external given forces through the real displacement. Hence, $H\left(x_{2}\right)$ denoting the (variable) height of the three-dimensional plate with the defect and $\mathbf{u}$ the three-dimensional displacement field at equilibrium, $\mathcal{P}^{\eta}$ reads

$$
\mathcal{P}^{\eta}=-\int_{-L / 2}^{L / 2} \int_{-\ell / 2}^{\ell / 2}\left(\int_{0}^{H\left(x_{2}\right)} f_{\mathrm{R}}(\mathbf{x}) u_{3}(\mathbf{x}) d x_{3}+F_{\mathrm{R}}\left(x_{1}, x_{2}\right) u_{3}\left(x_{1}, x_{2}, H\left(x_{2}\right)\right)\right) d x_{1} d x_{2},
$$

where the properties of symmetry have been used. After introducing the dimensionless quantities, one gets

$$
\mathcal{P}^{\eta}=-h L^{2} E \int_{\Omega_{0}}\left(\int_{0}^{\bar{H}\left(\bar{x}_{2}\right)} f\left(\overline{\mathbf{x}}, y_{3}\right) u_{3}^{\eta}\left(\overline{\mathbf{x}}, y_{3}\right) d y_{3}+F(\overline{\mathbf{x}}) u_{3}^{\eta}\left(\overline{\mathbf{x}}, \bar{H}\left(\bar{x}_{2}\right)\right)\right) d \overline{\mathbf{x}}
$$

where $\bar{H}\left(\bar{x}_{2}\right)=H\left(x_{2}\right) / h$. One can use the outer expansion (14) of $u_{3}^{\eta}$ everywhere but in the boundary layers where we must use the inner expansions. So by virtue of (15), (16) and (80), one gets

$$
\mathcal{P}^{\eta}=-\frac{1}{2} h L^{2} E\left(\int_{\Omega_{0}} \mathcal{F}^{0}\left(U^{0}+\eta U^{1}\right) d \overline{\mathbf{x}}+\eta \int_{I} \mathcal{F}_{0}^{0} U^{0} d \bar{x}_{1}\right)+\Delta \mathcal{P}^{\eta}
$$

where $\Delta \mathcal{P}^{\eta}$ denotes the real potential energy contained in the boundary layers diminished by the approximate potential energy contained in the boundary layers by using the outer expansion of $u_{3}^{\eta}$. (Specifically, neglecting the terms $o(\eta), \Delta \mathcal{P}^{\eta}$ is the integral over the boundary layer zones of the product of the external forces $f$ and $F$ by $u_{3}^{\eta}-U^{0}$.) Let us show that $\Delta \mathcal{P}^{\eta}$ is itself $o(\eta)$ by evaluating each boundary layer. We can take $\sqrt{\eta}$ as the thickness (at the scale of $\overline{\mathbf{x}}$ ) of the boundary layer because that corresponds to a distance from the side (or the defect) where the outer and inner expansions are both valid. Accordingly, it suffices to show that $u_{3}^{\eta}-U^{0}$ is of the order of $\eta$ in each boundary layer.

1. Near the clamped sides. There, since $v_{3}^{0}=v_{3}^{1}=0$, the contribution of $u_{3}^{\eta}$ to $\Delta \mathcal{P}^{\eta}$ is negligible. Moreover $U^{0}$ can be neglected in that boundary layer because $U^{0}=U_{, 1}^{0}=0$ at $\bar{x}_{1}= \pm 1 / 2$. Hence, $\Delta \mathcal{P}^{\eta}$ is negligible there.

2. Near the free side $\bar{x}_{2}=-\bar{\ell} / 2$. There using (41), (44) and (46), one gets

$$
U^{0}(\overline{\mathbf{x}})-u_{3}^{\eta}\left(\overline{\mathbf{x}}, y_{3}\right)=U^{0}\left(\bar{x}_{1}, \bar{x}_{2}\right)-U^{0}\left(\bar{x}_{1},-\bar{\ell} / 2\right)-U_{, 2}^{0}\left(\bar{x}_{1},-\bar{\ell} / 2\right)\left(\bar{x}_{2}+\frac{\bar{\ell}}{2}\right)+O(\eta)=O(\eta) .
$$

Therefore $\Delta \mathcal{P}^{\eta}$ is negligible at this side. By the same argument $\Delta \mathcal{P}^{\eta}$ is negligible near $\bar{x}_{2}=\bar{\ell} / 2$.

3. Near the defect. In the same manner, since $u_{3}^{\eta}\left(\overline{\mathbf{x}}, y_{3}\right)=U^{0}\left(\bar{x}_{1}, 0\right)+\bar{x}_{2} U_{, 2}^{0}\left(\bar{x}_{1}, 0\right)+O(\eta)$ in that zone, one concludes that $\Delta \mathcal{P}^{\eta}$ is negligible there. 
4. Near the corners $( \pm 1 / 2, \pm \bar{\ell} / 2)$ or the end points $\mathbf{D}^{ \pm}$. The inner expansions that we have considered for the three boundary layers are not valid near those points. We should consider another type of expansion to have a good approximation of the mechanical fields there. However, it is easy to show that, at first order, $u_{3}^{\eta}\left(\bar{x}, y_{3}\right)$ is equal to the value taken by $U^{0}$ at the corner (or the end point). That suffices to conclude that $\Delta \mathcal{P}^{\eta}$ is negligible in the neighbourhood of those points.

So we can conclude by

Proposition 6 The potential energy $\mathcal{P}^{\eta}$ of the plate at equilibrium can be evaluated up to second order by (90) which requires to determine both the regular and singular parts of $U^{1}$.

6.3 The merged model of plate including the boundary layer effects

The determination of $U^{1}$ requires to find first its singular part and then to solve a plate problem where the data depend in a rather intricate manner on the singularity and on the solution of the problem at the first order. From a practical point of view, it must be done on a case by case basis. For instance, singularities change when the end points of the defect are on a free side rather than on a clamped side, or when the angles at the corners of the plate between the clamped or the free sides are changed. To overcome these difficulties, the idea is to merge the two boundary value problems into a single problem which can be solved in one step and which does not contain any singularity. For that, reintroducing the small parameter $\eta$, one combines the first two problems in such a manner that $U^{0}+\eta U^{1}$ is a good approximation of the solution $U^{\eta}$ of that new problem (in fact the first two terms of the expansion). Specifically, the displacement field $U^{\eta}$ and the associated shear force and bending moment fields $\mathcal{T}^{\eta}$ and $\mathcal{M}^{\eta}$ are solutions of the following boundary value problem

\begin{tabular}{|c|c|c|}
\hline on $\partial_{c} \Omega_{0}$ & on $\partial_{f} \Omega_{0}$ & on $I$ \\
\hline $\begin{array}{l}U^{\eta}=0 \\
\mathrm{D} U_{, n}^{\eta}=\eta \mathrm{C}_{\mathrm{c}} \mathcal{M}_{n n}^{\eta},\end{array}$ & $\left\{\begin{array}{l}\mathcal{T}_{n}^{\eta}-\mathcal{M}_{n t, t}^{\eta}=0 \\
\mathcal{M}_{n n}^{\eta}=-6 \eta \mathrm{J}_{\mathrm{f}} \mathcal{M}_{n t, t}^{\eta}\end{array}\right.$ & $\left\{\begin{array}{l}\llbracket U^{\eta} \rrbracket=0, \\
\mathrm{D} \llbracket U_{, 2}^{\eta} \rrbracket=-\eta C_{\mathrm{d}}\left\langle\mathcal{M}_{22}^{\eta}\right\rangle, \\
\llbracket \mathcal{T}_{2}^{\eta}-\mathcal{M}_{21,1}^{\eta} \rrbracket=\eta \mathbf{l}_{\mathrm{d}} U_{, 1111}^{\eta}-\eta \mathcal{F}_{0}^{0}, \\
\llbracket \mathcal{M}_{22}^{\eta} \rrbracket=-6 \eta J_{\mathrm{d}}\left\langle\mathcal{M}_{21,1}^{\eta}\right\rangle,\end{array}\right.$ \\
\hline
\end{tabular}

where angle brackets stand for the average value on $I$, see (83). Le us compare this effective plate model to the Love-Kirchhoff plate. in (91) the usual boundary and transmission conditions are replaced by new ones. Specifically, on the clamped sides the displacement must still vanish, but its normal derivative which represents the rotation is no longer zero but proportional to the normal bending moment. In other words, the usual clamped condition is replaced by a Robin's type condition, the stiffness coefficient being of the order of $1 / \eta$. In the same manner, on the free sides one obtains also a condition linking the normal bending moment to the tangential derivative of the twist moment. Across the line of defect, one obtains jump condition of Ventcel's type $[2,9,15,29,35,46]$ where both the rotation, the shear forces and the moments can be discontinuous.

Let us give an energetic interpretation of this plate model and on the associated boundary and transmission conditions. That leads to the following proposition:

Proposition 7 The merged problem (91) is equivalent to find $U^{\eta}$ extremum of $\mathcal{E}^{\eta}$ over $\mathcal{V}$ where

$$
\begin{aligned}
& \mathcal{E}^{\eta}(V)=\int_{\Omega_{0} \backslash I} \frac{\mathrm{D}}{2}\left(\nu(\Delta V)^{2}+(1-\nu) \nabla^{2} V \cdot \nabla^{2} V\right) d \overline{\mathbf{x}}+\mathcal{B}^{\eta}(V)-\int_{\Omega_{0}} \mathcal{F}^{0} V d \overline{\mathbf{x}}-\int_{I} \eta \mathcal{F}_{0}^{0} V d \bar{x}_{1}, \\
\mathcal{B}^{\eta}(V)= & -\int_{\partial_{c} \Omega_{0}} \frac{\mathrm{D}}{2 \eta \mathrm{C}_{\mathrm{c}}}\left(V_{, n}\right)^{2} d \bar{x}_{2}-\int_{\partial_{f} \Omega_{0}} \frac{\eta \mathrm{J}_{\mathrm{f}}}{4(1+\nu)}\left(V_{, n t}\right)^{2} d \bar{x}_{1} \\
& -\int_{I} \frac{\mathrm{D}}{2 \eta \mathrm{C}_{\mathrm{d}}} \llbracket V_{, 2} \rrbracket^{2} d \bar{x}_{1}+\int_{I} \frac{\eta \mathrm{J}_{\mathrm{d}}}{4(1+\nu)}\left\langle V_{, 21}\right\rangle^{2} d \bar{x}_{1}+\int_{I} \frac{\eta \mathbf{I}_{\mathrm{d}}}{2}\left(V_{, 11}\right)^{2} d \bar{x}_{1}-\frac{\mathrm{I}_{\mathrm{d}}}{2 \mathrm{C}_{\mathrm{c}}}\left(V_{, 1}\left(\mathbf{D}^{+}\right)^{2}+V_{, 1}\left(\mathbf{D}^{-}\right)^{2}\right),
\end{aligned}
$$

and

$$
\mathcal{V}=\left\{V \in H^{2}\left(\Omega_{0} \backslash I\right) \cap H^{2}(I), \frac{\partial V}{\partial n} \in H_{0}^{1}\left(\partial_{f} \Omega_{0}\right),\left\langle\frac{\partial V}{\partial \bar{x}_{2}}\right\rangle \in H_{0}^{1}(I), V=0 \text { on } \partial_{c} \Omega_{0}, \llbracket V \rrbracket=0 \text { on } I\right\} .
$$

In the particular case where $\mathrm{C}_{\mathrm{d}}=0$, one must add the kinematic constraint $\llbracket V_{, 2} \rrbracket=0$ on $I$ in the definition of $\mathcal{V}$, and one must drop the associated integral over $I$ in the definition of $\mathcal{B}$. Similarly, in the particular case where $\mathrm{C}_{\mathrm{c}}=0$, one must add the kinematic constraint $V_{, 1}=0$ on $\partial_{c} \Omega_{0}$ in the definition of $\mathcal{V}$, and one must drop the associated integral over $\partial_{c} \Omega_{0}$ and the term at the end points $\mathbf{D}^{ \pm}$in the definition of $\mathcal{B}$. 
Proof Let us give a formal proof that if $U^{\eta}, \mathcal{M}^{\eta}$ and $\mathcal{T}^{\eta}$ satisfy (91), then $U^{\eta}$ is a stationary of $\mathcal{E}$ over the linear space $\mathcal{V}$. One starts from the equilibrium equation $\mathcal{M}_{\alpha \beta, \alpha \beta}^{\eta}=\mathcal{F}^{0}$ that is multiplied by $V \in \mathcal{V}$ and integrated over $\Omega_{0} \backslash I$. After two integrations by parts one gets

$$
\begin{aligned}
\int_{\Omega_{0} \backslash I} \mathcal{M}_{\alpha \beta}^{\eta} V_{, \alpha \beta} d \overline{\mathbf{x}}= & \int_{\Omega_{0}} \mathcal{F}^{0} V d \overline{\mathbf{x}}+\int_{\partial_{c} \Omega_{0}} \mathcal{M}_{n n}^{\eta} V_{, n} d \bar{x}_{2}+\int_{\partial_{f} \Omega_{0}}\left(\mathcal{M}_{n \alpha}^{\eta} V_{, \alpha}+\mathcal{T}_{n}^{\eta} V\right) d \bar{x}_{1} \\
& -\int_{I}\left(\llbracket \mathcal{M}_{22}^{\eta} V_{, 2} \rrbracket+\llbracket \mathcal{M}_{21}^{\eta} \rrbracket V_{, 1}+\llbracket \mathcal{T}_{2}^{\eta} \rrbracket V\right) d \bar{x}_{1} .
\end{aligned}
$$

Integrating by parts the terms $\mathcal{M}_{n t}^{\eta} V_{, t}$ on $\partial_{f} \Omega_{0}$ and $\llbracket \mathcal{M}_{21}^{\eta} \rrbracket V_{, 1}$ on $I$ leads to

$$
\int_{\Omega_{0} \backslash I}\left(\mathcal{M}^{\eta} \cdot \nabla^{2} V-\mathcal{F}^{0} V\right) d \overline{\mathbf{x}}=\int_{\partial_{c} \Omega_{0}} \mathcal{M}_{n n}^{\eta} V_{, n} d \bar{x}_{2}+\int_{\partial_{f} \Omega_{0}} \mathcal{M}_{n n}^{\eta} V_{, n} d \bar{x}_{1}-\int_{I} \llbracket \mathcal{M}_{22}^{\eta} V_{, 2} \rrbracket d \bar{x}_{1}-\int_{I} \llbracket \mathcal{T}_{2}^{\eta}-\mathcal{M}_{21,1}^{\eta} \rrbracket V d \bar{x}_{1}
$$

Let us simplify each integral in the right hand side of (92) with the help of the boundary conditions satisfied by $U^{\eta}, \mathcal{M}^{\eta}, \mathcal{T}^{\eta}$, and $V$.

1. For the first integral, if $C_{c}=0$ then $U_{, n}^{\eta}=0$ on $\partial_{c} \Omega_{0}$. Adding this kinematic constraint in the definition of $\mathcal{V}$, the integral vanishes. Otherwise, when $C_{c} \neq 0$, one gets from the boundary condition

$$
\int_{\partial_{c} \Omega_{0}} \mathcal{M}_{n n}^{\eta} V_{, n} d \bar{x}_{2}=\int_{\partial_{c} \Omega_{0}} \frac{\mathrm{D}}{\eta \mathrm{C}_{\mathrm{c}}} U_{, n}^{\eta} V_{, n} d \bar{x}_{2}
$$

2. For the second integral, boundary conditions give

$$
\int_{\partial_{f} \Omega_{0}} \mathcal{M}_{n n}^{\eta} V, n d \bar{x}_{1}=-\int_{\partial_{f} \Omega_{0}} 6 \eta J_{f} \mathcal{M}_{n t, t}^{\eta} V_{, n} d \bar{x}_{1}=\int_{\partial_{f} \Omega_{0}} 6 \eta \mathrm{J}_{\mathrm{f}} \mathcal{M}_{n t}^{\eta} V_{, n t} d \bar{x}_{1}=\int_{\partial_{f} \Omega_{0}} \frac{\eta \mathrm{J}_{\mathrm{f}}}{2(1+\nu)} U_{, n t}^{\eta} V_{, n t} d \bar{x}_{1},
$$

where we have used $V_{, n}=V_{, 2}=0$ at the corners of the plate.

3. For the third integral, using the general rule $\llbracket \phi \varphi \rrbracket=\llbracket \phi \rrbracket\langle\varphi\rangle+\langle\phi\rangle \llbracket \varphi \rrbracket$, one gets

$$
\int_{I} \llbracket \mathcal{M}_{22}^{\eta} V_{, 2} \rrbracket d \bar{x}_{1}=\int_{I}\left\langle\mathcal{M}_{22}^{\eta}\right\rangle \llbracket V_{, 2} \rrbracket d \bar{x}_{1}+\int_{I} \llbracket \mathcal{M}_{22}^{\eta} \rrbracket\left\langle V_{, 2}\right\rangle d \bar{x}_{1} .
$$

For the first integral in the right hand side above, if $C_{d}=0$ then $\llbracket U_{2}^{\eta} \rrbracket=0$ on $I$. Adding this kinematic constraint in the definition of $\mathcal{V}$, the integral vanishes. Otherwise, when $C_{d} \neq 0$, one gets from the boundary condition

$$
\int_{I}\left\langle\mathcal{M}_{22}^{\eta}\right\rangle \llbracket V, 2 \rrbracket d \bar{x}_{1}=-\int_{I} \frac{\mathrm{D}}{\eta \mathrm{C}_{\mathrm{d}}} \llbracket U_{, 2}^{\eta} \rrbracket \llbracket V_{, 2} \rrbracket d \bar{x}_{1}
$$

For the second integral, boundary conditions lead to

$$
\int_{I} \llbracket \mathcal{M}_{22}^{\eta} \rrbracket\left\langle V_{, 2}\right\rangle d \bar{x}_{1}=-\int_{I} 6 \eta J_{\mathrm{d}}\left\langle\mathcal{M}_{21,1}^{\eta}\right\rangle\left\langle V_{, 2}\right\rangle d \bar{x}_{1}=\int_{I} \frac{\eta J_{\mathrm{d}}}{2(1+\nu)}\left\langle U_{, 21}^{\eta}\right\rangle\left\langle V_{, 21}\right\rangle d \bar{x}_{1},
$$

where we have used $V=V_{, 2}=0$ at the end points $\mathbf{D}^{ \pm}$.

4. For the last integral, boundary conditions yield

$$
\int_{I} \llbracket \mathcal{T}_{2}^{\eta}-\mathcal{M}_{21,1}^{\eta} \rrbracket V d \bar{x}_{1}=\int_{I} \eta \mathbf{l}_{\mathrm{d}} U_{, 1111}^{\eta} V d \bar{x}_{1}-\int_{I} \eta \mathcal{F}_{0}^{0} V d \bar{x}_{1}
$$

After two integrations by parts, the first integral in the right hand side above becomes

$$
\int_{I} \eta \mathbf{l}_{\mathbf{d}} U_{, 1111}^{\eta} V d \bar{x}_{1}=\int_{I} \eta \mathbf{l}_{\mathbf{d}} U_{, 11}^{\eta} V_{, 11} d \bar{x}_{1}-\left.\eta \mathbf{l}_{\mathbf{d}} U_{, 11}^{\eta} V_{, 1}\right|_{\mathbf{D}^{-}} ^{\mathbf{D}^{+}}
$$

where we have accounted for $V=0$ at $\mathbf{D}^{ \pm}$. Let us evaluate the terms at $\mathbf{D}^{ \pm}$above. If $\mathbf{C}_{c}=0$, then $U_{, 1}^{\eta}=0$ on $\partial_{c} \Omega_{0}$ and hence at $\mathbf{D}^{ \pm}$. Inserting this kinematic constraint in the definition of $\mathcal{V}$, the terms at the end points vanish. Otherwise, since $U^{\eta}=U_{, 22}^{\eta}=0$ at $\mathbf{D}^{ \pm}$, one has $\eta U_{, 11}^{\eta}=\eta \mathcal{M}_{11}^{\eta} / \mathbf{D}= \pm U_{, 1}^{\eta} / \mathbf{C}_{\mathrm{c}}$ at $\mathbf{D}^{ \pm}$and hence the terms at $\mathbf{D}^{ \pm}$read

$$
\left.\eta \mathrm{I}_{\mathrm{d}} U_{, 11}^{\eta} V_{, 1}\right|_{\mathbf{D}^{-}} ^{\mathbf{D}^{+}}=\frac{\mathrm{I}_{\mathrm{d}}}{\mathrm{C}_{\mathrm{c}}}\left(U_{, 1}^{\eta}\left(\mathbf{D}^{+}\right) V_{, 1}\left(\mathbf{D}^{+}\right)+U_{, 1}^{\eta}\left(\mathbf{D}^{-}\right) V_{, 1}\left(\mathbf{D}^{-}\right)\right)
$$

Inserting the expression of the four integrals into (92), one easily sees that (92) is nothing but the vanishing of the first variation of $\mathcal{E}^{\eta}$. Hence $U^{\eta}$ is an extremal point of $\mathcal{E}^{\eta}$ in $\mathcal{V}$. One can prove the converse (at least formally) in the same manner. 
If one introduces the physical quantities in place of the dimensionless ones, the part $\mathcal{B}(V)$ of the energy associated with the boundary layers reads

$$
\begin{aligned}
\mathcal{B}(V) & =-\int_{x_{1}= \pm \frac{L}{2}} \frac{\mathrm{E} h^{2}}{24\left(1-\nu^{2}\right) \mathrm{C}_{\mathrm{c}}}\left(\frac{\partial V}{\partial x_{1}}\right)^{2} d x_{2}-\int_{x_{2}= \pm \frac{\ell}{2}} \frac{\mu h^{4} \mathrm{~J}_{\mathrm{f}}}{2}\left(\frac{\partial^{2} V}{\partial x_{2} \partial x_{1}}\right)^{2} d x_{1}-\left.\frac{\mathrm{E} h^{3} \mathrm{I}_{\mathrm{d}}}{2 \mathrm{C}_{\mathrm{c}}}\left(\frac{\partial V}{\partial x_{1}}\right)^{2}\right|_{( \pm L / 2,0)} \\
& +\int_{x_{2}=0}\left(-\frac{\mathrm{E} h^{2}}{24\left(1-\nu^{2}\right) \mathrm{C}_{\mathrm{d}}} \llbracket\left[\frac{\partial V}{\partial x_{2}} \rrbracket^{2}+\frac{\mu h^{4} \mathrm{~J}_{\mathrm{d}}}{2}\left(\frac{\partial}{\partial x_{1}}\left\langle\frac{\partial V}{\partial x_{2}}\right\rangle\right)^{2}+\frac{E h^{4} \mathrm{I}_{\mathrm{d}}}{2}\left(\frac{\partial^{2} V}{\partial x_{1}^{2}}\right)^{2}\right) d x_{1}\right.
\end{aligned}
$$

where $\mu$ denotes the shear modulus. Let us give an interpretation of each term of $\mathcal{B}$ and on its sign.

1. Since $\mathrm{C}_{\mathrm{c}}>0$ when $\nu \neq 0$, the energy associated with the boundary layer on the clamped sides is negative. If $\nu=0$, then $\mathrm{C}_{\mathrm{c}}=0$, there no longer is a boundary layer and that term disappears. When $\nu \neq 0$, the loss of bending energy is due to an increase of the stiffness induced by the clamping. The coefficient $C_{c}$ is a compliance modulus which measures this effect. The first term (the one associated with $C_{d}$ ) in the energy associated with the defect is interpreted in the same manner: when the defect is a geometrical stiffening, then the stiffener increases the flexural stiffness of the plate in the direction orthogonal to the stiffener what is traduced by $C_{d}>0$; on the other hand, when the defect is a geometrical weakening, the flexural stiffness of the plate in the orthogonal direction decreases and $C_{d}<0$. In the limit case where the defect separates the plate into two parts, then $C_{d}$ goes to minus infinity (see Figure 6 and 9 ) and there is no flexural rigidity in the direction $\mathbf{e}_{2}$.

2. The term with the coefficient $J_{d}$ in the energy associated with the defect corresponds to a torsional energy. When the defect is a geometrical weakening, then $J_{d}<0$, the plate loses this torsional energy, whereas when the defect is a geometrical stiffening, then $J_{d}>0$, the plate gains this torsional energy stored in the stiffener. When the defect is a crack, then $J_{d}<0$ and in the limit case where the crack separates the plate into two parts, then two free surfaces are created. In such a case one can see on Figure 9 that $J_{d}=-2 J_{f}$. Accordingly, the negative torsional energy associated with a free surface (term with $\mathrm{J}_{\mathrm{f}}$ ) is a particular case of loss of torsional energy induced by a geometrical weakening.

3. The term with the coefficient $I_{d}$ in the energy associated with the defect corresponds to a longitudinal bending energy of the defect which can be viewed as a beam. When the defect is a geometrical weakening, then $I_{d}<0$, the plate loses this bending energy, whereas when the defect is a geometrical stiffening, then $I_{d}>0$, the plate gains this bending energy stored in the beam. Note that the coefficient $I_{d}$ is the usual geometrical inertial moment that appears in the expression of the bending energy in the Navier-Bernoulli theory of beams. Here $I_{d}$ is negative, when material is removed from the plate.

Let us consider now the questions of existence and uniqueness of a solution to (91) or, equivalently, of an extremal point of $\mathcal{E}^{\eta}$ in $\mathcal{V}$. It is easy to check that if one searches a solution of (91) for $U^{\eta}$ close to $U^{0}$ then a such solution admits $U^{0}+\eta U^{1}$ as an expansion up to the second order (and the same property holds for the shear forces and the bending moments). That verification is left to the reader. But we are no longer ensured of the existence nor the uniqueness of a solution of (91). The natural space where one must search a solution is the one where all the terms involved in the energy $\mathcal{E}^{\eta}$ are finite. Such a space is more regular than $H^{2}\left(\Omega_{0} \backslash I\right)$ so that the torsional energies (terms with $J_{f}$ and $J_{d}$ ) and the longitudinal bending energy of the defect (terms with $I_{d}$ ) be defined. On the other hand, since several terms have the bad sign, coercivity of $\mathcal{E}^{\eta}$ over $\mathcal{V}$ is not ensured. Accordingly, one can no longer consider (91) as a minimization problem. In conclusion, the analysis of those questions must be refined, this task being outside the scope of this paper, is reserved to future works.

\section{Two examples of application}

We finish by two examples that illustrate the results obtained in the previous sections. The first one is the case of a reinforcement by two circular cylinders of radius $\mathrm{R}=\overline{\mathrm{R}} h$, one on each face of the plate. The second is the case of a weakening by two transverse cracks of depth $\mathrm{a}=\overline{\mathrm{a}} h$ symmetrically disposed across the thickness of the plate. That corresponds to the sections Y represented on Figure 8. 

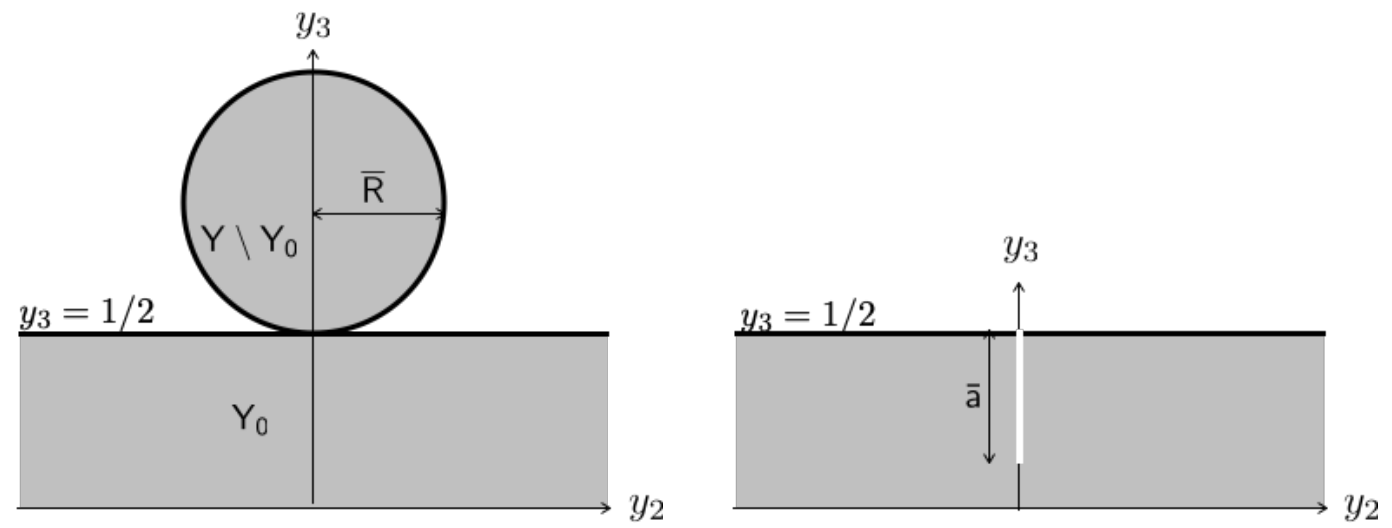

Fig. 8 Section $\mathrm{Y}$ of the defect in the two examples. Left: case of a stiffening by a circular cylinder; Right : case of a weakening by a transverse crack. By symmetry, there exists the same cylinder or the same crack in the opposite side $y_{3}<0$.

7.1 Case of a stiffening by two cylinders

The two boundary layer problems (67) and (68) can be solved in a closed form owing to the particularities of the geometry. Specifically, the stress field $\boldsymbol{\Sigma}^{\mathbf{d}}$ solution of the bending boundary value problem (67) reads

$$
\boldsymbol{\Sigma}^{\mathbf{d}}(\mathbf{y})= \begin{cases}\mathbf{0} & \text { in } \mathrm{Y}_{0}, \\ \frac{y_{3}}{1-\nu^{2}}\left(\nu \mathbf{e}_{1} \otimes \mathbf{e}_{1}+\mathbf{e}_{2} \otimes \mathbf{e}_{2}\right) & \text { in } \mathrm{Y} \backslash \mathrm{Y}_{0}\end{cases}
$$

It is the particular case where the coefficient $C_{d}$ vanishes, essentially because the attachment of the stiffener on the plate is reduced to a point. Therefore there is no modification of the transversal bending stiffness of the plate by the stiffener and one gets the kinematical constraint $\llbracket U_{, 2}^{1} \rrbracket=\llbracket U_{, 2}^{\eta} \rrbracket=0$ for the problems (84) or (91).

Since the section of the stiffener is circular and its link with the plate is reduced to a point, the displacement field $W^{d}$ solution of the twist boundary value problem (68) can also be obtained in a closed form and eventually reads

$$
\mathbf{W}^{\mathrm{d}}(\mathbf{y})= \begin{cases}0 & \text { in } \mathrm{Y}_{0} \\ y_{1}\left(y_{3}+\frac{1}{2}+\overline{\mathrm{R}}\right) & \text { in } \mathrm{Y} \backslash \mathrm{Y}_{0}\end{cases}
$$

One deduces that the coefficients $J_{d}$ and $I_{d}$ are given by

$$
\mathrm{J}_{\mathrm{d}}=\pi \overline{\mathrm{R}}^{4} \quad \mathrm{I}_{\mathrm{d}}=\frac{\pi}{2} \overline{\mathrm{R}}^{4}+\frac{\pi}{2} \overline{\mathrm{R}}^{2}(1+2 \overline{\mathrm{R}})^{2} .
$$

Thus, $J_{d}$ is twice the usual geometrical inertial moment of a circular disk. Accordingly, the part $\mathcal{B}_{\mathrm{d}}(V)$ of the energy $\mathcal{B}(V)$ associated with the stiffeners consists in the torsional and bending energies of the two cylinders seen as slender beams. Specifically, $\mathcal{B}_{\mathrm{d}}(V)$ reads

$$
\mathcal{B}_{\mathrm{d}}(V)=\int_{x_{2}=0}\left(\frac{\mu}{2} h^{4} \mathrm{~J}_{\mathrm{d}} V_{, 21}^{2}+\frac{\mathrm{E}}{2} h^{4} \mathrm{I}_{\mathrm{d}} V_{, 11}^{2}\right) d x_{1}
$$

\subsection{Case of a weakening by two transverse cracks}

The two boundary layer problems (67) and (68) must be solved numerically. Using a finite element code one obtains the values of the two coefficients $C_{d}$ and $J_{d}$ for any value of the crack depth $\bar{a}$. Since the crack has no thickness, the coefficient $I_{d}$ vanishes. The graphs of $J_{d}$ and $C_{d}$ in function of $\bar{a}$ are plotted on Figure 9 for $\nu=0.25\left(C_{d}\right.$ depends on $\nu$, not $\mathrm{J}_{\mathrm{d}}$ ). Both coefficients are negative, in conformity with Proposition 4, and decreasing functions of $\overline{\mathrm{a}}$. As expected, when ā grows from 0 to $1 / 2$, the compliance factor $C_{d}$ decreases from 0 to $-\infty$ whereas the torsional modulus $\mathrm{J}_{\mathrm{d}}$ decreases from 0 to $-2 \mathrm{~J}_{\mathrm{f}}$. 

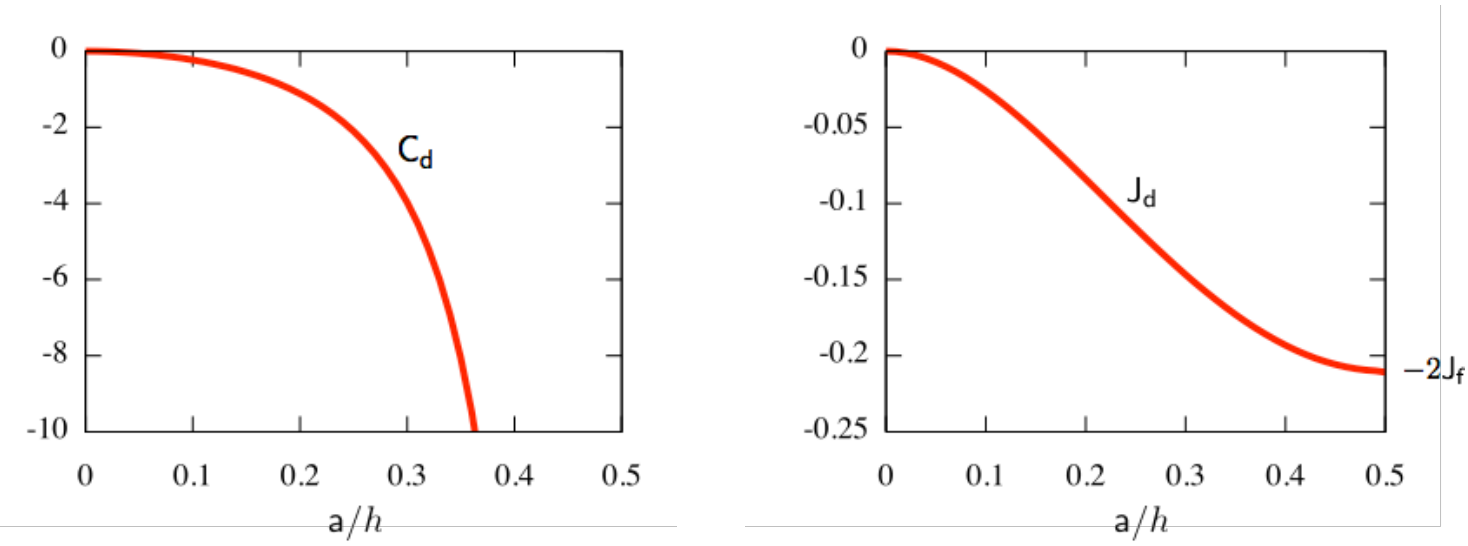

Fig. 9 Graphs of the coefficients $C_{d}$ and $J_{d}$ as a function of the length a of the crack when $\nu=0.25$. They respectively represent effective rotational and torsional stiffnesses.

Those results have been obtained assuming that the defect is invariant in the direction $x_{1}$ and hence that the depth of the crack is a constant independent of $x_{1}$. Nevertheless the results remain true even if the depth of the crack depends of $\bar{x}_{1}$ provided that this dependence is smooth. The effect of the gradient of $\bar{x}_{1} \mapsto \overline{\mathrm{a}}\left(\bar{x}_{1}\right)$ will appear at higher orders but not in the problem (84) giving $U^{1}$. Accordingly, one can still use all the results of the previous subsections with $\bar{a}$ depending smoothly on $\bar{x}_{1}$. In this context, we propose to give the expression of the potential energy release rate $\mathcal{G}=-\delta \mathcal{P}^{\eta} / \delta S$ associated with a virtual increase $\delta S=h L \int_{I} \delta \overline{\mathrm{a}}\left(\bar{x}_{1}\right) d \bar{x}_{1}$ of the cracked surface from the uniform depth $\overline{\mathrm{a}}_{0}$. Since $U^{0}$ does not depend on the depth of the crack, $U^{0}$ and all the first order terms do not change when the depth is changed. Only $U^{1}$ and all the second order terms will change. Denoting by $\delta U^{1}$ the variation of $U^{1}$, by virtue of Proposition 6 and the fact that the loading is kept constant, $\mathcal{G}$ can be approximated by

$$
\mathcal{G}=\frac{\mathrm{E} h}{2} \frac{\int_{\Omega_{0}} \mathcal{F}^{0}(\overline{\mathbf{x}}) \delta U^{1}(\overline{\mathbf{x}}) d \overline{\mathbf{x}}}{\int_{I} \delta \overline{\mathbf{a}}\left(\bar{x}_{1}\right) d \bar{x}_{1}}
$$

Since $U^{0}, C_{c}$ and $J_{f}$ do not depend on $\overline{\mathrm{a}}\left(\bar{x}_{1}\right)$, and since $\mathrm{I}_{\mathrm{d}}=0$, one deduces from (84) that the variation $\delta U^{1}$ of $U^{1}$ and the associated variations $\delta \mathcal{M}^{1}$ and $\delta \mathcal{T}^{1}$ of the bending moments and the shear forces are solution of the following boundary value problem

$$
\text { in } \Omega_{0} \backslash I:\left\{\begin{array}{l}
\operatorname{div} \delta \mathcal{T}^{1}=0, \quad \operatorname{div} \delta \mathcal{M}^{1}+\delta \mathcal{T}^{1}=0, \\
\delta \mathcal{M}_{\alpha \beta}^{1}=\mathrm{D}\left(\nu \Delta \delta U^{1} \delta_{\alpha \beta}+(1-\nu) \delta U_{, \alpha \beta}^{1}\right)
\end{array}\right.
$$

$$
\begin{aligned}
& \text { on } \partial_{c} \Omega_{0}:\left\{\begin{array}{l}
\delta U^{1}=0, \\
\delta U_{, n}^{1}=0,
\end{array} \quad \text { on } I:\left\{\begin{array}{l}
\llbracket \delta U^{1} \rrbracket=0, \\
\llbracket \delta \mathcal{T}_{2}^{1}-\delta \mathcal{M}_{21,1}^{1} \rrbracket=0, \\
\mathrm{D} \llbracket \delta U_{, 2}^{1} \rrbracket=-\delta \mathrm{C}_{\mathrm{d}} \mathcal{M}_{22}^{0}, \\
\llbracket \delta \mathcal{M}_{22}^{1} \rrbracket=-6 \delta \mathrm{J}_{\mathrm{d}} \mathcal{M}_{21,1}^{0},
\end{array}\right.\right. \\
& \text { on } \partial_{f} \Omega_{0}:\left\{\begin{array}{l}
\delta \mathcal{T}_{n}^{1}-\delta \mathcal{M}_{n t, t}^{1}=0, \\
\delta \mathcal{M}_{n n}^{1}=0,
\end{array}\right.
\end{aligned}
$$

where

$$
\left.\delta \mathrm{C}_{\mathrm{d}}\left(\bar{x}_{1}\right)=\frac{d \mathrm{C}_{\mathrm{d}}}{d \overline{\mathrm{a}}}\left(\overline{\mathrm{a}}_{0}\right) \delta \overline{\mathrm{a}}\left(\bar{x}_{1}\right), \quad \delta \mathrm{J}_{\mathrm{d}}\left(\bar{x}_{1}\right)=\frac{d \mathrm{~J}_{\mathrm{d}}}{d \overline{\mathrm{a}}}\right)\left(\overline{\mathrm{a}}_{0}\right) \delta \overline{\mathrm{a}}\left(\bar{x}_{1}\right)
$$

By the same arguments as those used for the problem (84), one deduces that the solution of $(94)$ is unique, $\delta U^{1}$ having the same type of singularity as $U^{1}$. However, its determination is not necessary to obtain $\mathcal{G}$ as we show hereafter. Starting from the equilibrium equations satisfied by $U^{0}$ and $\delta U^{1}$, one gets

$$
\int_{\Omega_{0}} \mathcal{F}^{0} \delta U^{1} d \overline{\mathbf{x}}=\int_{\Omega_{0} \backslash I}\left(\mathcal{M}_{\alpha \beta, \alpha \beta}^{0} \delta U^{1}-\delta \mathcal{M}_{\alpha \beta, \alpha \beta}^{1} U^{0}\right) d \overline{\mathbf{x}} .
$$

Integrating by parts twice the right hand side above, using all the boundary conditions, the smoothness of $U^{0}$ and the equality $\mathcal{M}_{\alpha \beta}^{0} \delta U_{, \alpha \beta}^{1}=\delta \mathcal{M}_{\alpha \beta}^{1} U_{, \alpha \beta}^{0}$, one eventually obtains

$$
\int_{\Omega_{0}} \mathcal{F}^{0} \delta U^{1} d \overline{\mathbf{x}}=-\int_{I}\left(\frac{\delta \mathrm{C}_{\mathrm{d}}}{\mathrm{D}}\left(\mathcal{M}_{22}^{0}\right)^{2}+6 \delta \mathrm{J}_{\mathrm{d}} \mathcal{M}_{21}^{0} U_{, 21}^{0}\right) d \bar{x}_{1}
$$


Inserting this latter relation into (93) allows us to write the potential energy release rate under the following form

$$
\mathcal{G} \int_{I} \delta \overline{\mathrm{a}}\left(\bar{x}_{1}\right) d \bar{x}_{1}=\mathrm{E} h \int_{I} \mathrm{G}\left(\bar{x}_{1}\right) \delta \overline{\mathbf{a}}\left(\bar{x}_{1}\right) d \bar{x}_{1},
$$

with

$$
\mathrm{G}\left(\bar{x}_{1}\right)=-6\left(1-\nu^{2}\right) \frac{d \mathrm{C}_{\mathrm{d}}}{d \overline{\mathrm{a}}}\left(\overline{\mathrm{a}}_{0}\right) \mathcal{M}_{22}^{0}\left(\bar{x}_{1}, 0\right)^{2}-36(1+\nu) \frac{d \mathrm{~J}_{\mathrm{d}}}{d \overline{\mathrm{a}}}\left(\overline{\mathrm{a}}_{0}\right) \mathcal{M}_{21}^{0}\left(\bar{x}_{1}, 0\right)^{2} .
$$

Thus, $\mathrm{G}\left(\bar{x}_{1}\right)$ corresponds to the dimensionless local energy release rate and, as expected, is positive. If one adopts the Griffith energy criterion, $\mathrm{G}\left(\bar{x}_{1}\right)$ allows us to evaluate the risk of propagation of the crack at the point $\bar{x}_{1}$ of the line of defect by comparing it with the surface energy density $G_{c}$. To that end, it is sufficient to calculate the bending moment field along the axis $\bar{x}_{2}=0$ with the usual Love-Kirchhoff theory (and hence as if the plate did not contain any defect) and to use the two functions giving the coefficients $C_{d}$ and $J_{d}$ in function of the depth of the crack. Since those coefficients only depend on the defect and not on the overall geometry of the plate nor on its loading, the analysis of the risk of propagation is both possible (unlike to Love-Kirchhoff model) and greatly simplified by comparison to a $3 \mathrm{D}$ approach.

\section{Conclusion and perspectives}

In the present paper an asymptotic method was developed to study boundary layer effects on the sides of a thin elastic plate and to account for the presence of a geometrical defect inside the plate. The analysis results in a bending model of plate which improves the classical Love-Kirchhoff one (in a different manner than the MindlinReissner model). Indeed, it consists either in solving successively two boundary value problems, or a single merged one. In the first approach, the first boundary value problem is the classical Love-Kirchhoff model which does not account for boundary layer effects but gives the data to solve the second boundary value problem which, in turn, accounts for these effects. The particularity of the second boundary value problem is that its solution is in general singular, requiring a careful study of the singularities on a case by case basis. In the second approach, the two boundary value problems are merged into a single one which no longer contains any singularity but whose mathematical properties remain to be well established. In both approaches, the new model allows us to treat the question of the propagation of transverse cracks in an asymptotic manner without coming back to a threedimensional approach. This enhanced model is based on new "effective" boundary or transmission conditions of Robin's or Ventcel's type which simply require solving once and for all a few boundary layer problems to obtain the coefficients entering in these effective conditions. From an energetic point of view, the merged problem contains additional terms in the expression of the effective potential energy of the plate. These terms correspond to surface energies associated to boundary layers and some of them have the particularity of being negative.

Even if the construction of the enhanced model has been made in a particular context where are considered only clamped or free boundary conditions, straight and one-directional geometrical defects, and pure bending responses, the method could be easily adapted and the results extended to more general situations. Let us list some possible or desirable extensions that could be the subject of future works: (i) consider the general threedimensional problem which leads to a model of plate coupling bending and membrane responses; (ii) complete the analysis of the boundary layers by a comprehensive study of "all" usual boundary conditions, including curvilinear frontiers; (iii) consider geometrical defects more general than those symmetric, straight, and one-directional; (iv) complete the mathematical analysis of the merged boundary value problem.

\section{References}

1. Abdelmoula, R., Coutris, M., Marigo, J.J.: Comportement asymptotique d'une interphase élastique mince. C. R. Acad. Sci. Paris Série II b 326(4), 237-242 (1998)

2. Abdelmoula, R., Marigo, J.J.: The effective behavior of a fiber bridged crack. J. Mech. Phys. Solids 48(11), 2419-2444 (2000)

3. Abels, H., Mora, M.G., Müller, S.: Large time existence for thin vibrating plates. Commun. Part. Diff. Eq. 36, 2062-2102 (2011)

4. Alessandrini, S., Arnold, D., Falk, R., Madureira, A.: Derivation and justification of plate models by variational methods. Plates and Shells 21 (1999). DOI 10.1090/crmp/021/01

5. Batra, R.C., Vidoli, S.: Higher-order piezoelectric plate theory derived from a three-dimensional variational principle. AIAA J. 40(1), 91-103 (2002)

6. Bessoud, A.L., Krasucki, F., Michaille, G.: Multi-materials with strong interface: variational modelings. Asymptotic Anal. 61(1), 1-19 (2009)

7. Bessoud, A.L., Krasucki, F., Serpilli, M.: Asymptotic analysis of shell-like inclusions with high rigidity. J. Elasticity 103(2), 153-172 (2011)

8. Bilteryst, F., Marigo, J.J.: An energy based analysis of the pull-out problem. Eur. J. Mech. - A/Solids 22(1), 55-69 (2003)

9. Bonnaillie-Noël, V., Dambrine, M., Hérau, F., Vial, G.: On generalized Ventcel's type boundary conditions for Laplace operator in a bounded domain. SIAM J. Math. Anal. 42(2), 931-945 (2010) 
10. Ciarlet, P.: Mathematical Elasticity, Vol. II: Theory of Plates. Studies in Mathematics and its Applications. Springer, NorthHolland, Amsterdam (1997)

11. Ciarlet, P.G., Destuynder, P.: A justification of the two-dimensional linear plate model. J. Mécanique 18, 315-344 (1979)

12. Dauge, M., Gruais, I., Rössle, A.: Influence of lateral boundary conditions on the asymptotics in thin elastic plates. SIAM J. Math. Anal. 31(2), 305-345 (1999)

13. Dauge, M., Tordeux, S., Vial, G.: Selfsimilar perturbation near a corner: matching versus multiscale expansions for a model problem. In: Around the research of Vladimir Maz'ya. II, Int. Math. Ser. (N. Y.), vol. 12, pp. 95-134. Springer (2010)

14. Dauge, M., Yosibash, Z.: Boundary layer realization in thin elastic three-dimensional domains and two-dimensional hierarchic plate models. International Journal of Solids and Structures 37(17), $2443-2471$ (2000). DOI https://doi.org/10.1016/S00207683(99)00004-9. URL http://www.sciencedirect.com/science/article/pii/S0020768399000049

15. David, M., Marigo, J.J., Pideri, C.: Homogenized interface model describing inhomogeneities localized on a surface. J. Elasticity 109(2), 153-187 (2012)

16. Dumontet, H.: Homogénéisation et effets de bords dans les matériaux composites. Thèse de doctorat d'État. Université Pierre et Marie Curie, Paris (1990)

17. Eckhaus, W.: Matched Asymptotic Expansions and Singular Perturbations. No. 6 in Mathematics studies. North-Holland (1973)

18. Fosdick, R., Royer Carfagni, G.: Singular shear-force states in elementary plate theory. Journal of Elasticity 118 (2015). DOI 10.1007/s10659-014-9480-7

19. Galuppi, L., Royer Carfagni, G.: On the occurrence of lumped forces at corners in classical plate theories. a physically-based interpretation. Journal of Mechanics of Materials and Structures 10 (2015). DOI 10.2140/jomms.2015.10.93

20. Geymonat, G., Hendili, S., Krasucki, F., Vidrascu, M.: The matched asymptotic expansion for the computation of the effective behavior of an elastic structure with a thin layer of holes. Int. J. Multiscale Com. (2011)

21. Green, A.E.: Boundary-layer equations in the linear theory of thin elastic shells. Proc. R. Soc. Lond. A 269, 481-491 (1962)

22. Grisvard, P.: Singularités en élasticité. Arch. Rat. Mech. Anal. 107, 157-180 (1989)

23. Häggblad, B., Bathe, K.J.: Specifications of boundary conditions for Reissner/Mindlin plate bending finite elements. Int. J. Num. Methods Engin. 30, 981-1011 (1990)

24. Hanna, N.F., Leissa, A.W.: A higher order shear deformation theory for the vibration of thick plates. J. Sound Vibration $\mathbf{1 7 0}(4), 545-555(1994)$

25. Huy, H.P., Sanchez-Palencia, E.: Phénomène de transmission à travers des couches minces de conductivité élevée. J. Math. Anal. Appl. 47, 284-309 (1974)

26. Ilin, A.M.: Matching of Asymptotic Expansions of Solutions of Boundary Value Problems. Translations of Mathematical Monographs, Vol. 102. American Mathematical Society (1992). URL http://gen.lib.rus.ec/book/index.php?md5=81e53d8fa4f7e02c3148ca215fe40cf8

27. Karamian, P., Sanchez-Hubert, J.: Boundary layers in thin elastic shells with developable middle surface. Eur. J. Mech. A/Solids 21, 13-47 (2002)

28. Lagerstrom, P.: Matched Asymptotic Expansions: Ideas and Techniques. No. 76 in Applied mathematical sciences. Springer Verlag (1988)

29. Lemrabet, K.: Le problème de Ventcel pour le système de l'élasticité dans un domaine de $\mathbb{R}^{3}$. C. R. Acad. Sci. Paris Série I 304(6), 151-154 (1987)

30. Lobkovsky, A.E.: Boundary layer analysis of the ridge singularity of a thin plate. Phys. Rev. E 53(4), 3750-3759 (1996)

31. Love, A.E.H.: On the small free vibrations and deformations of elastic shells. Philos. T. R. Soc. A A(17), 491-549 (1888)

32. Ma, H.M., Gao, X.L., Reddy, J.N.: A non-classical mindlin plate model based on a modified couple stress theory. Acta Mech. 220, 217-235 (2011)

33. Marigo, J.J., Maurel, A.: Second order homogenization of subwavelength stratified media including finite size effect. SIAM J. Appl. Math. 77(2), 721-743 (2017)

34. Marigo, J.J., Maurel, A., Pham, K., Sbitti, A.: Effective dynamic properties of a row of elastic inclusions: The case of scalar shear waves. J. Elasticity 128(2), 265-289 (2017)

35. Marigo, J.J., Pideri, C.: The effective behavior of elastic bodies containing microcracks or microholes localized on a surface. Int. J. Damage Mech. 20, 1151-1177 (2011)

36. Mindlin, R.D.: Influence of rotatory inertia and shear on flexural motions of isotropic, elastic plates. J. Appl. Mech. 18, 31-38 (1951)

37. Naghdi, P.M.: The theory of shells and plates. In: S. Flügge, C. Truesdell (eds.) Handbuch der Physik, vol. VIa/2, pp. 425-640. Springer-Verlag (1972)

38. Pham, K., Maurel, A., Marigo, J.J.: Two scale homogenization of a row of locally resonant inclusions-the case of anti-plane shear waves. J. Mech. Phys. Solids 106, 80-94 (2017)

39. Poisson, M.: Memoire sur l'equilibre et le mouvement des corps élastiques. Mémoires de l'Académie des Sciences de l'Institut de France (1828)

40. Reddy, J.N.: A simple higher-order theory for laminated composite plates. J. Appl. Mech. 51, 745-752 (84)

41. Reissner, E.: The effect of transverse shear deformation on the bending of elastic plates. J. Appl. Mech. 12, 68-77 (1945)

42. Timoshenko, S., Woinowsky-Krieger, S.: Theory of plates and shells. McGraw-Hill, New York (1959)

43. Touratier, M.: An efficient standard plate theory. Int. J. Engng Sci. 29(8), 901-916 (1991)

44. Vasil'ev, V.: Kirchhoff and thomson-tait transformations in the classical theory of plates. Mechanics of Solids 47 (2012). DOI 10.3103/S0025654412050111

45. Vasil'ev, V.: Torsion of a square isotropic plate by forces applied at the corners and by distributed torques. Mechanics of Solids 52, 134-143 (2017). DOI 10.3103/S0025654417020030

46. Ventcel, A.D.: On boundary conditions for multi-dimensional diffusion processes. Theor. Probability Appl. 4, 164-177 (1959) 